\title{
Excessive alcohol use in youth on holiday : an evaluation of two intervention methods
}

Citation for published version (APA):

van de Luitgaarden-Janssen, J. (2009). Excessive alcohol use in youth on holiday : an evaluation of two intervention methods. [Doctoral Thesis, Maastricht University]. Datawyse / Universitaire Pers Maastricht. https://doi.org/10.26481/dis.20090320jl

Document status and date:

Published: 01/01/2009

DOI:

10.26481/dis.20090320jl

Document Version:

Publisher's PDF, also known as Version of record

\section{Please check the document version of this publication:}

- A submitted manuscript is the version of the article upon submission and before peer-review. There can be important differences between the submitted version and the official published version of record.

People interested in the research are advised to contact the author for the final version of the publication, or visit the DOI to the publisher's website.

- The final author version and the galley proof are versions of the publication after peer review.

- The final published version features the final layout of the paper including the volume, issue and page numbers.

Link to publication

\footnotetext{
General rights rights.

- You may freely distribute the URL identifying the publication in the public portal. please follow below link for the End User Agreement:

www.umlib.nl/taverne-license

Take down policy

If you believe that this document breaches copyright please contact us at:

repository@maastrichtuniversity.nl

providing details and we will investigate your claim.
}

Copyright and moral rights for the publications made accessible in the public portal are retained by the authors and/or other copyright owners and it is a condition of accessing publications that users recognise and abide by the legal requirements associated with these

- Users may download and print one copy of any publication from the public portal for the purpose of private study or research.

- You may not further distribute the material or use it for any profit-making activity or commercial gain

If the publication is distributed under the terms of Article $25 \mathrm{fa}$ of the Dutch Copyright Act, indicated by the "Taverne" license above, 


\section{Excessive alcohol use in youth on holiday}

An evaluation of two intervention methods 
The studies presented in this thesis were performed at the Care and Public Health Research institute (CAPHRI) of Maastricht University. CAPHRI participates in the Netherlands School of Primary Care Research (CaRe), re-acknowledged by the Royal Dutch Academy of Science (KNAW) in 2000.

The Netherlands Organisation for Health Research and Development (ZonMw; grant number 4010.0001) is gratefully acknowledged for funding the studies in this thesis.

\section{ISBN 9789052788128}

Printed by Datawyse, Universitaire Pers Maastricht

Cover Design by Priscilla Brouwer, Maastricht

Copyright (C) 2009, J. van de Luitgaarden, Maastricht

All rights reserved. No part of this thesis may be reprot

in any form or by any means, electronic, prior written permission of the author, mechanical, photocopying, recording, or otherwise, without articles. 


\title{
Excessive alcohol use in youth on holiday An evaluation of two intervention methods
}

\author{
PROEFSCHRIFT \\ ter verkrijging van de graad van doctor aan de Universiteit Maastricht, \\ op gezag van de Rector Magnificus, \\ Prof. Mr. G.P.M.F. Mols \\ volgens het besluit van het College van Decanen, \\ in het openbaar te verdedigen \\ op vrijdag 20 maart 2009 om 12.00 uur \\ door \\ Jade van de Luitgaarden - Janssen
}

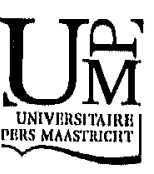




\section{Promotores}

Prof. dr. R.A. Knibbe

Prof. dr. R.W. Wiers (Universiteit van Amsterdam)

\section{Beoordelingscommissie}

Prof. dr. N.K. de Vries (voorzitter)

Prof. dr. G.J. Kok

Dr. P.H. Lemmens

Prof. dr. H.D. van de Mheen (Erasmus Universiteit Rotterdam)

Prof. dr. W.A.M. Vollebergh (Universiteit Utrecht) 


\section{CONTENTS}

Chapter 1 Introduction 7

Chapter 2 Prevention of alcohol problems in Dutch youth: Missed 21 opportunities and new developments

Chapter 3 Challenging implicit and explicit alcohol-related cognitions in 35 young heavy drinkers

Chapter 4 From the laboratory to real-life: A pilot study of an expectancy challenge with "heavy drinking" young people on holiday

Chapter 5 Single-session expectancy challenge with young heavy drinkers on holiday

Chapter 6 How easy is it to get drunk? Contextual factors related to young people's excessive alcohol use on holiday

Chapter 7 Adolescents binge drinking when on holiday: An evaluation of a 103 community intervention based on self regulation

Chapter 8 General Discussion

Samenvatting

Dankwoord 139

Curriculum Vitae

Publications 
CHAPTER 1

Introduction 
In the Netherlands, over $10 \%$ of people aged $16-69$ are problem drinkers. Among 16-25 year olds this number is even higher: $22 \%$ (Van Dijck \& Knibbe, 2005). Alcohol consumption and frequency of intoxication has increased in Dutch adolescents over the past decades (Monshouwer, van Dorsselaer, Gorter, Verdurmen, \& Vollebergh, 2004). Young people typically have their first alcoholic drink when they are between 11 and 15 years old, and frequency of consumption increases with age (Monshouwer, Verdurmen, van Dorsselaer, Smit, Gorter, \& Vollebergh, 2008). Dutch young people are now the most frequent drinkers of Europe (Hibell et al., 2004). Binge drinking (> 5 drinks per occasion) occurs often, especially during the weekend when nearly half of 16 year olds drink five or more alcoholic drinks (Monshouwer et al., 2008). Boys drink more often than girls (Monshouwer et al., 2008).

Alcohol use often decreases when people reach their twenties and take on the roles and responsibilities of adulthood (Bachman et al., 2002; Hajema \& Knibbe, 1998). However, alcohol abuse is one of the main causes of morbidity and mortality during adolescence and young adulthood in both the Netherlands and the rest of Europe (Anderson \& Baumberg, 2006).

There is one particular period in which many adolescents and young adults drink much more than they regularly do. On holiday, young men often drink as much as 22 standard drinks a day, during a one to two-week period (Lemmers, Willems, Thijssen, \& Osterman, 1998). This pattern of excessive drinking causes several problems, such as black-outs, fights, alcohol poisonings, traffic accidents, and unsafe sex (Newcomb \& Bentler, 1989). Alcohol use is exacerbated during this time, due to several factors: absence of parents, presence of a large group of (male) friends, and the feeling of being in a "time-out" situation, in which normal behavioral rules do not apply, and school and parents are far away (Pos, Knibbe, \& Lemmers, 2001). With no one to supervise them, no social responsibilities and few activities to be done, drinking is the main pastime.

The Dutch "National Institute for Disease Prevention and Health Promotion" (NIGZ) has attempted an interpersonal intervention for many years in vacation communities. Although youth were more aware of the risks of excessive alcohol use after the intervention (Lemmers et al., 1998), their alcohol consumption did not decrease. Therefore, Maastricht University and NIGZ started a project to test two intervention methods in this population. The primary aim of this study was to develop and evaluate these two intervention methods, specifically community interventions (CI) and expectancy challenges (EC).

The rest of this chapter will be devoted to the design of the main study, an overview of expectancy challenge and community intervention research, and a description of the aims and outlines of the thesis.

\subsection{Design of the main study}

At the onset, a design with 4 conditions was planned for the main study (see Table 1). However, during the course of the project a suitable "community intervention only" municipality proved difficult to recruit. The community that was eventually 
chosen (Zandvoort) dropped out of the study after it became clear that local support for the intervention was limited and that the purpose of the community intervention was not sufficiently in line with local problems. Therefore, the main study included Schouwen-Duiveland $(n=191)$ as a community in which both expectancy challenges and a community intervention were carried out. Terschelling $(n=178)$ was included as a community in which only expectancy challenges took place, and Texel $(n=86)$ was added as a control community. The community intervention on Schouwen-Duiveland, and the Expectancy Challenges on Schouwen-Duiveland and Terschelling took place in July and August of 2004. Data collection for the main study also took place during these months. Subjects were recruited on camping sites by trained peers (both female and male) and asked to take part in an alcohol study. Participants who took part in the Expectancy Challenge were driven to the intervention location shortly afterwards. Only young males were approached to participate $(N=492$, mean age 17.9 years, minimum 16.0 years; the minimum legal drinking age). Most of them were on holiday with a group of friends. Of each group of friends, only one participant was recruited.

Table 1. Design of the main study

Expectancy Challenge
\begin{tabular}{|l|l|}
\hline $\begin{array}{l}\text { Expectancy Challenge } \\
\text { AND } \\
\text { Community Intervention }\end{array}$ & Community Intervention \\
\hline Expectancy Challenge & No intervention \\
\hline
\end{tabular}

With the original study design it would have been possible to analyze if any synergetic effects took place if a community intervention was combined with an Expectancy Challenge, as well as test the effect of a community intervention alone.

\subsection{Community interventions}

One approach for reducing alcohol and other drug problems is community-based prevention programs. These focus on changing the environment in which a person consumes alcohol rather than on persuading individuals to change their behaviors. Generally, the supply of alcohol through social and commercial sources is targeted, as opposed to the demand for alcohol by individuals. Environmental approaches focus on the community as a system involving numerous components, including individual drinkers, vendors of alcohol, social events where alcoholic beverages are sold and consumed, local regulations and enforcement practices, local substance abuse services, and social organizations that promote healthy living. Comprehensive community interventions that involve more than one entity (e.g., police and alcohol sellers), take place in a variety of settings (e.g., camping site and bars), and are 
maintained for a longer period have the potential to positively affect the population that is targeted. Little is known about the intervention components that are involved in the success of a community intervention. Most community interventions have several working principles in common: First, participation of members of the community in decision-making on programs that influence health (Wandersman \& Florin, 2000); Second, intersectoral collaboration, where resources of stakeholders are brought together to solve problems which cannot be solved by one stakeholder alone (Gray, 1985); Third, using positive network forces to promote healthy behavior (Heany \& Israel, 2002); and Fourth, connecting to what already exists, thus building on what is already present in a community (Thompson \& Kinne, 1999).

Community interventions for health promotion should follow five phases according to Bracht, Kingsbury and Rissel's community organization model (1999): 1. Community analysis, in which information about the community is collected, and local organizations and actors are drawn into the project, 2. Design and initiation, in which the project starts and a first design of the project is made, 3. Implementation, in which a combination of interventions is designed and implemented, which together, contribute to achieving the goals of the project, 4 . Maintenance and consolidation, to ensure continuation of the community intervention method, and 5 . Reorientation, in which the total project is reconsidered and a new community analysis takes place.

Studies show that environmental interventions aimed at preventing youth access to alcohol can aid in reducing youth alcohol abuse (Wagenaar, Toomey, \& Erickson, 2005; Stafström, Östergren, Larsson, Lindgren, \& Lundborg, 2006). General measures, such as raising the minimum drinking age to 21 , can decrease prevalence of youth alcohol consumption (O'Malley and Wagenaar, 1991). Studies of taxes on alcohol (Grossman, Coate, and Arluck, 1987; Levy and Sheflin, 1985) and the licensing of establishments that sell alcohol (Holder and Blose, 1987; Wagenaar and Holder, 1991) also indicate that policies that increase the price of alcohol or limit its availability reduce consumption of alcohol and problems associated with alcohol use (George et al., 1989; O'Malley and Wagenaar, 1991). In addition, interventions that reduce alcohol availability can reduce alcohol related fatal traffic crashes (Hingson, Zakocs, Heeren, Winter, Rosenbloom, DeJong, 2005).

Internationally, projects have also included measures taken in a local context, such as stricter enforcement of underage alcohol sales laws through increasing age checks in bars (e.g. Holder, Saltz, Grube, Treno, Reynolds, Voas, \& Gruenewald, 1997), limiting the numbers and locations of alcohol outlets in the community (Grube and Nygaard, 2001; Holder et al., 1997), training management of alcohol establishments, reducing the availability of alcohol from personal sources who are over 21, and changing cultural norms that tolerate underage drinking (e.g. Wagenaar, Murray, Wolfson, Forster, \& Finnegan, 1994).

Several comprehensive community intervention projects on alcohol are well known, for example, "Project Northland", a community-wide intervention designed to reduce adolescent alcohol use. The program includes six years of programming and is multi-level, involving education of teens and parents, providing positive peer pressure, implementing media campaigns, and forming community action teams 
that aim to reduce commercial and social access to alcohol. Several beneficiary effects were found. At the end of the third year of the intervention, students reported less use of alcohol in both the past week and the past month. At the end of the sixth year, the ability to purchase alcohol in off-sale outlets was reduced, and parents in intervention communities had less permissive norms regarding teen alcohol use as compared to parents in control communities (Perry, Williams, Komro, Veblen-Mortenson, Stigler, Munson, Farbakhsh, Jones, \& Forster, 2002).

The Trelleborg project is another promising community project aimed at preventing adolescent alcohol use. It included interventions that complemented the school program, a community policy and action plan on alcohol and drugs, increased inspection of local shops by police to check for sales of alcohol to underage youth, a parents' information program, and news coverage of the adolescent surveys of alcohol and drug use in the community. The project resulted in a decrease in heavy episodic drinking by 15 - and 16-year-old youth over a 4-year period (19992003). This result is especially remarkable because overall Swedish alcohol consumption increased in this period (Strafstöm et al., 2006).

Another program is "Communities that Care" ( Hawkins \& Catalano, 1992). It is a comprehensive, community-wide risk-focused prevention strategy that aims to reduce risk factors that predict adolescent problem behaviors (including alcohol use) and increase protective factors that inhibit these behaviors.

In the Netherlands, the present project was the first attempt to apply the community intervention approach to heavily drinking youth on holiday. SchouwenDuiveland volunteered to take part as a study community, and was a suitable candidate because of its issues with youth drinking excessively when vacationing there. The community intervention implemented in that community is different from those done in other countries in two ways: 1 . It was aimed at temporary residents (youth on holiday), and 2. Participation of relevant actors (e.g. shop and pub owners, camping attendants) was on a voluntary basis with little or no means to enforce measures agreed upon. Chapter 7 details the results of this intervention.

\subsection{Expectancy Challenges}

Laboratory research using the balanced placebo design (Marlatt \& Rosenhow, 1980) has demonstrated that consumption of alcohol (or placebo) does not only result in pharmacological effects; cognitive variables also play a role in observed effects after alcohol consumption (Goldman, Brown, \& Christiansen, 1987). One important predictor of alcohol consumption is constituted by the expectancies about the effects of alcohol that a person has (Darkes \& Goldman, 1993; Wiers, Gunning, \& Sergeant, 1998). A person's expectancies can be regarded as knowledge on the relationship between objects and events in the real world. Based on one's expectancies, a person can anticipate on systematic relations between events and objects in future situations. Expectancies can be present implicitly or explicitly.

Explicit alcohol expectancies are cognitions that one is aware of and that can be measured with questionnaires. On the other hand, implicit cognitions are unavaila- 
ble to self-report or introspection (Greenwald \& Banaji, 1995) and can only be measured with indirect tests, such as the implicit association test (IAT; Greenwald, McGhee, \& Schwartz, 1998). The IAT is designed to examine which words and concepts are strongly paired in people's minds. For example, "bath" is associated with "soap" rather than with "lamp". It is harder to associate bath with lamp than with soap. Connecting concepts that the mind perceives as incompatible simply takes extra time. The difference in reaction time can be quantified and is an objective measure of people's implicit attitudes.

Expectancies about effects that alcohol will bring about can be distinguished in 3 different categories: 1) Positive reinforcement, such as increased sociability, assertiveness, fun, and sex, 2) Negative reinforcement, i.e. tension reduction, and 3) Negative expectancies, e.g. lower self-confidence, cognitive and motor problems, negative mood (Goldman \& Darkes, 2004). The first two categories are both essentially positive expectancies.

Many studies have shown correlations between these types of expectancies and alcohol consumption (Goldman, Del Boca \& Darkes, 1999). Positive and negative reinforcement expectancies are associated with current alcohol use (Brown et al., 1985; Sher et al., 1991). However, such cross-sectional studies cannot demonstrate causality. Longitudal studies are better suited to this. Longitudinal studies indicate that alcohol expectancies can predict future alcohol consumption up to 9 years later (Christiansen et al., 1989; Stacy, Newcomb, \& Bentler, 1991) in a variety of age groups and drinking populations (Leigh \& Stacy, 2004). However, the strongest evidence for a causal link could come from studies that tested if changes in alcohol use were mediated by changes in expectancies. Before the start of this study, this had not been done.

Apart from the positive-negative dimension, alcohol expectancies can be placed on a arousal-sedation dimension (Rather \& Goldman, 1994). One can make a distinction between expectations of arousal (e.g. funny, energetic, horny) and sedation (e.g. sleepy, intoxicated, woozy, Goldman et al., 1999). Lighter drinkers hold more sedation expectancies, while heavy drinkers hold more arousal expectancies. The idea behind the Expectancy Challenge (Darkes \& Goldman, 1993) is to change one's expectancies in such a way that it leads to a change in alcohol consumption. If successful in practice, the procedure could demonstrate the causal role of expectancies, as well as offer an intervention method for heavy drinkers.

In a typical Expectancy Challenge session (EC; Darkes \& Goldman, 1993) young people are brought together and receive a glass of vodka-tonic or a placebo drink. A game (Pictionary) is played while the drinks are consumed. After the game is finished, all participants are asked to write down who they think has had alcohol. Judgments are made on the basis of subjects' behavior (e.g. funny, outgoing, loud). Usually, many drinkers are wrongly identified as non-drinkers and vice versa. These discrepancies are used to discuss expectancies in the context of social or sexual situations. Furthermore, the differences between physical and expectancy effects of alcohol are emphasized.

Using a three-session EC protocol on male university students $(N=79)$, Darkes and Goldman (1993) found significant reductions in positive expectancies and alcohol 
consumption two weeks after the intervention, as compared to controls who received traditional education, or assessment-only. Since then, many studies using the EC have been conducted. Some studies applied the EC to women as well (e.g. Corbin, McNair, \& Carter, 2001; Dunn, Lau, \& Cruz, 2000; Musher-Eizenman \& Kulick, 2003), other studies reduced the number of sessions in the EC from three to one (e.g. Corbin et al., 2001). Furthermore, some 'information-only' ECs were done, in which no actual alcohol was consumed (Corbin et al., 2001; L. Jones, Silvia, \& Richman, 1995).

Dunn, Lau, \& Cruz (2000) were the first to replicate Darkes and Goldman's results in men. In their sample of men $(n=19)$ and women $(n=19)$ they found a reduction in social expectancies in both sexes. Male participants reduced their alcohol consumption as a result of the EC, but women did not. Musher-Eizenman \& Kulick (2003) applied the EC to at-risk college women. The program changed some expectancies, but did not reduce alcohol consumption. However, in a small sample, Wiers and Kummeling (2004) succeeded in reducing young women's positive alcohol expectancies and alcohol consumption with a multiple-session EC, while they found no changes in men. Furthermore, a recent study by Wood, Capone, Laforge, Erickson, \& Brand (2007) found reductions in alcohol use and heavy episodic drinking after the EC in both men and women. And Lau-Barraco \& Dunn (2008) found that exposure to an EC intervention led to significant decreases in alcohol expectancies and subsequent alcohol consumption in both males and females at follow-up. Although for years $E C^{\prime}$ 's appeared to be more successful in changing men's alcohol consumption than women's, it seems that in light of recent studies that notion may need to be corrected.

Most EC studies have used multiple-session protocols, however in some studies the number of sessions was reduced to one (e.g. Corbin, McNair, \& Carter, 2001). Multi-session ECs may result in larger reductions in positive-arousal expectancies and alcohol consumption than single-session protocols, however single-session ECs are easier conducted as an intervention in real-life settings.

Furthermore, some studies used EC protocols that did not include administrating alcoholic or placebo drinks (e.g. Corbin, McNair, \& Carter, 2001; Jones, Sylvia, \& Richman, 1995). The advantage of such an "information-only" EC is that it could be used during treatment of alcoholics. Unfortunately, information-only EC's have not succeeded in decreasing alcohol consumption.

Conclusions about the effectiveness of the $\mathrm{EC}$ are hampered by differences in protocols used, small sample sizes, relatively brief follow-up periods, and a lack of mediation analyses (Jones et al., 2001).

This thesis details the development of the EC from a laboratory experiment (Chapter 3 ) to an intervention method that can be used in real-life situations with youth on holiday (Chapter 4 and 5). 


\subsection{Alcohol expectancies and environmental factors}

Little is known about the relationship between alcohol expectancies and environmental factors. It is possible that expectancies cause one to select a certain environment, that in turn contributes to a certain level of alcohol use. Although it is also possible that environmental factors trigger specific expectancies, which may lead to alcohol use. However, environmental factors and expectancies may also both affect alcohol consumption completely independently from each other. Figure 1 provides a possible model of the relation between environmental factors, expectancies, and alcohol use. In the model, "environment" is a very broad term, that includes very diverse factors, such as group of friends, law enforcement, alcohol prices, opening hours of bars, etc. Arrow 1 outlines how the environment contributes to the development of alcohol expectancies. In our study, participants had already formed alcohol expectancies. Arrow 2 depicts the relation between alcohol expectancies and alcohol consumption, which was discussed previously under the heading "Expectancy Challenge". Arrow 3 a describes the way that these environmental factors influence the effect that alcohol expectancies have on drinking behavior. For example, alcohol expectancies may be of less influence on drinking behavior in a person that visits a bar in a community where alcohol laws are strict and well enforced, as opposed to a community where alcohol is available to anyone at anytime. Arrow $3 \mathrm{~b}$ describes how environmental factors directly influence drinking behavior, independently from alcohol expectancies. For example, a person that visits a bar is likely to stop drinking when he's out of money. This will happen earlier if he's drinking in a place were alcohol prices are high, than if he's drinking somewhere where alcohol prices are low. In effect, if alcohol prices are low, he is likely to drink more.

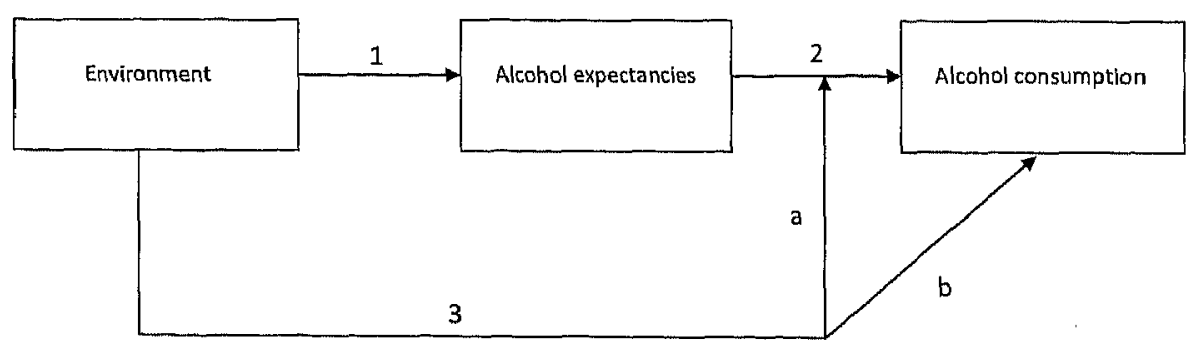

Figure 1. A model of the relationship between environmental factors, expectancies, and alcohol use

Research into the ways in which environmental factors, expectancies and alcohol use are inter-related would be very welcome, however the focus of this thesis is primarily on effectiveness of measures, and not so much on the theory that lies behind. 


\subsection{Aims and outline of this thesis}

Alcohol use in youth and problem drinking by adults has been identified by the Dutch government as one of the main issues in the public health sector (Ministry of Public Health, Welfare, \& Sports, 2006). Because young people drink particularly much when they are on holiday, it seems important to target drinking behavior in this situation. The target group of our study therefore consisted of heavy drinking young people on holiday. This thesis describes studies evaluating the feasibility and effectiveness of two interventions, that can be used in this context, namely community interventions and expectancy challenges.

This thesis consists of six articles, whose main objectives are summarized below.

Chapter 2 aims to provide a literature-based analysis of the current Dutch situation of youth alcohol use, and give insight into factors that hinder alcohol prevention in the Netherlands, as well as identify promising initiatives and changes that may hold promise for the future (Chapter 2). Attention is given to cultural factors that influence youth drinking, alcohol policies, enforcement of alcohol laws, interventions, as well as to the current political climate for prevention of alcohol problems.

Chapter 3 aims to evaluate if a single-session EC is effective in reducing university students' $(N=92)$ alcohol use through changing alcohol expectancies. In particular, the effects of the intervention on arousal and sedation expectancies and alcohol use were studied. It was hypothesized that arousal expectancies would decrease, while sedation expectancies would increase due to participation in the EC. Furthermore, it was hypothesized that expectancies would mediate alcohol use. This study took place in the university's bar lab.

Chapter 4 aims to assess the feasibility of a single-session EC in a real-life prevention setting, with youth on holiday. In this study $(N=170)$ the Expectancy Challenge was translated from the bar lab into an actual prevention environment. Furthermore, effects of the EC on arousal and sedation alcohol expectancies were tested, as well as on alcohol use during and after the vacation. Gender differences were also explored.

Chapter 5 aims to test the effectiveness of an optimized single-session EC in a larger sample of young men on holiday. Again, we were interested if changes in explicit alcohol expectancies and alcohol use would occur both during the holiday and after the holiday, as compared to a control group who did not take part in the EC. Additionally, it was assessed if any effect on alcohol use was mediated by a change in expectancies.

Chapter 6 aims to assess contextual factors related to young men's alcohol consumption on holiday. A stepwise regression analysis was used to determine the relevance of these factors in a sample of 492 young men on vacation.

Chapter 7 aims to evaluate compliance and effects of a community intervention based on self-regulation of stakeholders. The community intervention was initiated on Schouwen-Duiveland, a community that attracts a large number of young, heavily drinking tourists every year. To battle youth alcohol abuse in the tourist season, a 
voluntary covenant which included several measures was signed. We were interested in compliance with these measures, and in effects that were brought about by the efforts of stakeholders.

Chapter 8 provides an overview and general discussion of the main results of the studies in this thesis. Additionally, strengths and limitations of this thesis will be discussed, as well as implications and recommendations for alcohol prevention practice. Directions for further studies will also be explored. 


\section{References}

Anderson, P. \& Baumberg, B. (2006). Alcohol in Europe. Institute of Alcohol Studies.

Bachman, J.G., O'Malley, P.M., Schulenberg, J.E.; et al. (2002). The Decline of Substance Use in Young Adulthood: Changes in Social Activitles, Roles, and Beliefs. Mahwah, NJ: Lawrence Erlbaum Associates.

Bracht, N., Kingsbury, L., and Rissel, C. (1999). "A Five Stage Community Organization Model for Health Promotion: Empowerment and Partnership Strategies." In Health Promotion at the Community Level: New Advances, ed. N. Bracht. Thousand Oaks, CA: Sage Publications.

Brown, S.A. (1985). Expectancies versus background in the prediction of college drinking patterns. Journal of Consulting and Clinical Psychology, 53(1), 123-130.

Brown, S.A., Christiansen, B.A., \& Goldman, M.S. (1987). Journal of Studies on Alcohol, 48, 483-491.

Chassin, L., Pitts, S.C., Prost, J. (2002). Binge drinking trajectories from adolescence to emerging adulthood in a high-risk sample: Predictors and substance abuse outcomes. Journal of Consulting and Clinical Psychology, 70(1), 67-78.

Christiansen, B.A., Smith, G.T., Roehling, P.V., \& Goldman, M.S. (1989). Using alcohol expectancies to predict adolescent drinking after one year. Journal of Consulting and Clinical Psychology, 57, 93-99.

Corbin, W.R., MCNair, L.D. \& Carter, J.A. (2001). Evaluation of a treatment-appropriate cognitive intervention for challenging alcohol outcome expectancies. Addictive Behaviors, 26, 475 - 488.

Darkes, J. \& Goldman, M. S. (1993). Expectancy challenge and drinking reduction: experimental evidence for a mediational process. Journal of Consulting and Clinical Psychology, 61, 344-353.

Dunn, M. E., Lau, H. C. \& Cruz, I. Y. (2000). Changes in activation of alcohol expectancies in memory in relation to changes in alcohol use after participation in an expectancy challenge program. Experimental and Clinical Psychopharmacology, 8, 566-575.

George, W.H., Crowe, L.C., Abwender, D., \& Skinner, J.B. (1989). Effects of raising the drinking age to 21 years in New York state on self-reported consumption by college students. Journal of applied social psychology, 19(8), 623-635.

Goldman, M.S. \& Darkes, J. (2004) Alcohol Expectancy Multiaxial Assessment: A Memory Network-Based Approach. Psychological Assessment, v16 n1, 4-15.

Goldman, M. S., Del Boca, F. K. \& Darkes, J. (1999). Alcohol expectancy theory: the application of cognitive neuroscience. In H. T. Blane, \& K. E. Leonard (Eds.), Psychological theories of drinking and alcoholism (2nd ed.). pp. 203-246. NY: Guilford.

Gray, B. (1985). Conditions facilitating interorganizational collaboration. Human Relations, 38, 911-936.

Greenwald, A.G. \& Banaji, M.R.(1995). Implicit Social Cognition: Attitudes, Self-Esteem, and Stereotypes. Psychological Review, 102(1), 4-27.

Greenwald, A. G., McGhee, D. \& Schwartz, J. L. K, (1998). Measuring individual differences in implicit cognition: The Implicit Association Test. Journal of Personality and Social

Psychology, 74, 1464-1480

Grossman, M., Coate, D., Arluck, G.M. (1987). Price sensitivity of alcoholic beverages in the United States: Youth alcohol consumption. In: Holder, H. \{ed.\}, Control issues in Alcohol Abuse Prevention: Strategies for States and Communities. Greenwich, CT: JAI Press, pp. 169-198.

Grube, J.W. \& Nygaard, P. (2001). Adolescent drinking and alcohol policy. Contemporary Drug Problems, 28, 87-131.

Hajema, K.J. \& Knibbe, R.A. (1998), Changes in social roles as predictors of changes in drinking behaviour. Addiction, 93, 1717-1727.

Hawkins, J.D.; Catalano, R.F.; and Associates. (1992). Communities That Care, San Francisco, CA: JosseyBass Publishers.

Heany, C.A., \& Israel, B.A. (2002). Social networks and social support. In K.Glanz \& F.M. Lewis \& B.K. Rimer (Eds.), Health behavior and health education. Theory, research and practice (pp 185-209). San Francisco: Jossey-Bass. 
Hibell B., Andersson B., Bjarnason T., Ahlström S., Balakireva O., Kokkevi A., et al. (2004). The ESPAD Report 2003; Alcohol and Other Drug use Among Students in 35 European Countries, Stockholm: The Swedish Council for Information on Alcohol and Other Drugs (CAN) and the Pompidou Group at the Council of Europe.

Hingson, R.W., Zakocs, R.C., Heeren, T., Winter, M.R., Rosenbloom, D., \& DeJong, W. (2005). Effects on alcohol related fatal crashes of a community based initiative to increase substance abuse treatment and reduce alcohol availability. Injury prevention, 11, 84-90.

Holder, H.D., \& Blose, J.O.(1987). Impact of Changes in Distilled Spirits Availability on Apparent Consumption: a time series analysis of liquor-by-the-drink. British Journal of Addiction, 82 (6) , 623-631.

Holder, H.D., Saltz, R.F., Grube, J.W., Treno, A.J., Reynolds, R.I., Voas, R.B., \&

Gruenewald, P.J. (1997) Summing up: lessons from a comprehensive community prevention trial. Addiction, 92, 293-302.

Jones, B. T., \& McMahon, J. (1994). Negative alcohol expectancy predicts post-treatment abstinence survivorship: The whether, when and why of relapse to a first drink. Addiction, 89, 1653-1665.

Jones, B.T., Corbin, W. \& Fromme, K. (2001). A review of expectancy theory and alcohol consumption. Addiction, 91, 57-72.

Jones, L., Silvia, L. \& Richman, C.L (1995). Increased awareness and self-challenge of alcohol expectancles. Substance Abuse, 16, 77-85.

Leigh, B.C. \& Stacy, A.W. (2004). Alcohol expectancies and drinking in different age groups. Addiction, 99, 215-227

Lemmers, L., Willems, Y., Thijssen, P., \& Osterman, C. (1998). Evaluatie zomercampagne 1997 Lazer op

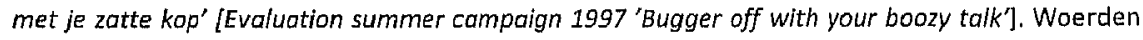
NIGZ.

Levy, D., and Sheflin, N. (1985). The demand for alcoholic beverages: An aggregate time-series analysis. Journal of Public Policy and Marketing, 4, 47-54.

Marlatt, G. A. \& Rohsenow, D. J. (1980). Cognitive processes in alcohol use: expectancy and the balanced placebo design. in N. K. Mello (Ed.), Advances in substance abuse: behavioral and biological research. pp. 159-199. Greenwich: JAl Press.

Ministry of Public Health, Welfare, \& Sports (2006). Preventienota Kiezen voorGezond Leven [Government amendment 'Choosing a healthy life'].(Parliamentary document No. PG/OGZ/2.720.104). Retrieved June 9, 2007, from http://www.minvw5.nl/images/preventienota_tcm19-137692,pdf

Monshouwer, K., Dorsselaer, S. Van, Gorter, A., Verdurmen, J. et al. (2004). Jeugd en Riskant Gedrag. Kerngegevens uit het peilstationsonderzoek 2003. Roken, drinken, drugsgebruik en gokken onder scholieren vanaf tien jaar [Youth and risky behavior. Core data 2003. Smoking, drinking, drug use, and gambling in pupils over ten years old]. Utrecht: Trimbos-institute.

Monshouwer, K., Verdurmen, J., Dorsselaer, S., Smit, E., Gorter, A., Vollebergh, W. (2008). Jeugd en riskant gedrag 2007. Kerngegevens uit het Peilstationonderzoek Scholieren. Roken, drinken, drugsgebruik en gokken onder scholieren vanaf tien jaar [Youth and risky behavior 2007. Core data an smoking, drinking, drug use, and gambling in pupils over ten years old]. Utrecht: Trimbos-institute,

Musher-Eizenman, D. R. \& Kulick, A. D. (2003). An alcohol expectancy-challenge prevention program for at-risk college women. Psychology of Addictive Behaviors, 17, 163-166.

Newcomb, M.D. \& Bentler, P.M. (1989). Substance Use and Abuse among Children and Teenagers. American Psychologist, 44(2), 242-48.

O'Malley, P.M. and Wagenaar, A.C. (1991). Effects of minimum drinking age laws on alcohol use, related behaviors and traffic crash involvement among American youth: 1976-1987. Journal of Studies on Alcohol, 52(5), 478-491.

Poikolainen, K., Tuulia-Henriksson, A., Aalto-Setälä, T., Marttunen, M., \& Lönnqvist, J. (2001). Predictors of alcohol intake and heavy drinking in early adulthood: a 5-year follow-up of 15-19-year-old Finnish adolescents. Alcohol and Alcoholism 36(1), 85-88. 
Perry, Williams, Komro, Veblen-Mortenson, Stigler, Munson, Farbakhsh, Jones, \& Forster (2002). Project Northland: long-term outcomes of community action to reduce adolescent alcohol use. Health Education Research, 17(1), 117-132.

Pos, S., Knibbe, R. \& Lemmers, L. (2001), Alle dagen feest? Een kwalitatieve studie naar omgevingsinvioeden op het alcoholgebruik van jongeren op vakantie [Party everyday? A qualitative study of environmental influences on young people's alcohol use on holiday]. Woerden/Maastricht: NIGZ/UM.

Rather, B.C., \& Goldman, M.S. (1994). Drinking-related differences in the memory organization of alcohol expectancies. Experimental and Clinical Psychopharmacology, 2, 167-183.

Sher, K. J., Walitzer, K.S., Wood, P.K., Brent, E.E. (1991). Characteristics of children of alcoholics: Putative risk factors, substance use and abuse, and psychopathology. Journal of Abnormal Psychology, 100(4), $427-448$

Stacy, A.W., Newcomb, M.D., \& Bentier, P.M. (1991). Cognitive motivation and drug use: A 9-year longitudinal study. Journal of Abnormal Psychology, 100, 502-515.

Stafström, M., Östergren, P., Larsson, S., Lindgren, B., Lundborg, P. (2006). A community action programme for reducing harmful drinking behaviour among adolescents: the Trelleborg Project. Addiction, 101, 813-823.

Thompson, B., \& Kinne, S. (1999). Social Change Theory. Applications to community health. In N. Bracht (Ed.), Health promotion at the community level 2-New advances (pp.29-58). Thousand Oaks: Sage.

Van Dijck, D. \& Knibbe, R.A. (2005). De prevalentie van probleemdrinken in Nederland IThe prevalence of problem drinking in The Netherlands]. Maastricht: UM.

Wagenaar, A.C. \& Holder, H.D. (1991). A change from public to private sale of wine: results from natural experiments in lowa and West Virginia. Journal of Studies on Alcohol, 52(2), 162-73.

Wagenaar, A. C., Murray, D. M., Wolfson, M., Forster, J. L., \& Finnegan, J. R. (1994). Communities Mobilizing for Change on Alcohol: Design of a randomized trial. Journal of Community Psychology, 22(CSAP Special Issue), 79-101.

Wagenaar, A.C., Toomey, T.L., Erickson, D.J. (2005). Preventing youth access to alcohol: Outcomes from a multi-community time-series trial. Addiction, 100, 335-345.

Wandersman, A. \& Florin, P. (2000), Citizen participation and community organizations. In J. Rappaport \& E.Seidman (Eds.). Handbook of community psychology (pp. 247-272). New York: Kluwer Academic/Plenum Publishers.

Wiers, R.W., Gunning, W.B., Sergeant, J.A. (1998) Do Young Children of Alcoholics Hold More Positive or Negative Alcohol-Related Expectancies Than Controls? Alcoholism: Clinical and Experimental Research $22(8), 1855-1863$.

Wiers R. W. \& Kummeling R. C. (2004). An Experimental Test of An Alcohol Expectancy Challenge in Mixed Gender Groups of Young Heavy Orinkers. Addictive Behaviors, 29, 215-220.

Wood, M.D., Capone, C., Laforge, R., Erickson, D.J., \& Brand, N.H. (2007). Brief motivational intervention and alcohol expectancy challenge with heavy drinking college students: A randomized factorial study. Addictive Behaviors, 32, 2509-2528. 


\section{CHAPTER 2}

\section{Prevention of alcohol problems in Dutch youth: Missed opportunities and new developments}




\begin{abstract}
Binge drinking among young people is a problem in the Netherlands. This paper outlines the current Dutch approach to alcohol prevention in this target group. It is argued that well enforced evidence-based control measures are lacking, despite renewed political interest in them. Politicians often favour alcohol education, but to increase the effectiveness of alcohol prevention a combined approach of policy measures, enforcement and education is needed. Translation of education and policy-based measures is discussed.
\end{abstract}




\subsection{Introduction}

Research has shown that excessive alcohol consumption is associated with a large array of short- and long term consequences, including black-outs, fights, alcohol poisonings, traffic accidents, unsafe sex, school drop-out, unemployment, depression, and brain damage (Newcomb \& Bentler, 1989). In addition, recent brain research has indicated that excessive alcohol and drug use during adolescence has much stronger consequences than during adulthood. Brain function is affected more as a result of adolescent alcohol abuse (Dahl \& Spear, 2004).

Over $10 \%$ of Dutch people aged 16-69 are problem drinkers, and among 16-25 year olds this number is even higher: $22 \%$ (Van Dijck \& Knibbe, 2005). Alcohol consumption and frequency of intoxication has increased in Dutch adolescents over the past decades, and more young girls are drinking alcohol (Monshouwer, van Dorsselaer, Gorter, Verdurmen, \& Vollebergh, 2004). Dutch young people are now the most frequent drinkers of Europe (Hibell et al., 2004). Over 30\% percent of Dutch twelve-year olds report having drank alcohol in the past month, and this number increases steadily for older adolescents ( $43 \%$ of thirteen year olds, $62 \%$ of fourteen year olds, $70 \%$ of fifteen year-olds, Monshouwer et al., 2004). Binge drinking occurs often, especially among young men during the weekend (Monshouwer et al., 2004) and on holiday (Van de Luitgaarden, Wiers, Knibbe, \& Boon, 2006). As a result, the number of alcohol poisonings among 12-17 year olds is rising (Van Kleef \& Van der Lely, 2006).

Drinking often starts at a peer's home and continues into the evening in bars and discos, with friends, where over $75 \%$ of total consumption takes place (Knibbe et al., 2007). Beer is relatively cheap in the Netherlands (World Health Organization [WHO], 2004) and is the most popular drink among young men (Trimbos Institute, 2007). In the past few years, sweet, alcohol-containing drinks ("alcopops") have become popular among youth, especially among girls (Monshouwer et al., 2004).

This article will focus on alcohol prevention in adolescents and young adults in the Netherlands, and the degree to which interventions that have a solid international research base are translated into effective measures in this country. We will begin by giving some background information on the Netherlands and outlining the history of alcohol there. Then the liberal cultural attitude regarding adolescent drinking will be discussed as well as alcohol policies in the Netherlands and preventive efforts that are undertaken. Finally, we will argue which measures are needed and discuss the cultural and political hurdles that are met when trying to adapt Dutch alcohol prevention to be more evidence-based.

\subsection{The Netherlands}

The Netherlands are a parliamentary democracy, but also a constitutional monarchy with the parliament bearing responsibility for the country's policy, rather than the Monarch. Along social-democratic lines, the Dutch government assumes responsibi- 
lity for the welfare of its citizens by being involved in matters relating to health care, education, employment, and social security. Using Hofstede's dimensions (1991), the Netherlands can be described as being an uncertainty-tolerant individualist country, with a small power distance, and a nurturing culture. The country's tolerant attitude is also demonstrated by the fact that it is legal to run a brothel, to marry a same-sex partner, and to commit euthanasia or abortion (under certain conditions). Furthermore, the sale, possession and consumption of small quantities of cannabis, while technically still illegal, is officially tolerated by the authorities. Recently, the Dutch government identified five areas in the public health sector that will be given extra funding for prevention efforts. Along with obesity, diabetes, smoking, and depression, alcohol use by youth under 16 and problem drinking in adults is one of the main issues (Ministry of Public Health, Welfare, \& Sports, 2006a).

\subsubsection{History of Alcohol}

Alcohol has been produced and consumed for thousands of years in Europe. Well into the nineteenth century an ambivalent image of alcohol was present in the Netherlands: moderate consumption was considered positive or healthy, however abuse was considered condemnable. During the time of the French occupation (1795 - 1815) consumption of Dutch gin increased. The temperance societies first campaigned against Dutch gin only, but in the second half of the nineteenth century, all alcoholic beverages came under suspicion (Van Amerongen, 1988). In 1881, the first 'liquor law' was passed. Since then, alcohol laws have been gradually extended. The condemnation of alcoholic beverages themselves, which was very strong in the $19^{\text {th }}$ and early $20^{\text {th }}$ century, has been slowly replaced by the present general opinion that alcohol is a substance with rather positive effects if used recreationally and in moderation (van Amerongen, 1988).

\subsubsection{Cultural Aspects of Adolescent Drinking}

In many cultures alcohol is perceived as having a social function; it is thought to increase sociability (Heath, 1995). This is also the case in the Netherlands (Wiers, van Woerden, Smulders, \& de Jong, 2002). Of course, negative consequences (crimes, aggression etc.) are also attributed to alcohol consumption, but these are expected to happen mainly after drinking copious amounts. Young men aged 16-24 years hold distinctly more positive expectancies for drinking a larger amount of alcohol than the general population (Wiers, Hoogeveen, Sergeant, \& Gunning, 1997). This may be due to the fact that adolescents experience the positive arousal effects of drinking more than the negative sedative effects (National Institute on Alcohol Abuse and Alcoholism [NIAAA], 2005).

Drinking large amounts of alcohol while going out during the weekend is part of youth culture in the Netherlands (and in many other countries, Engels \& Knibbe, 1999). In the Randstad (the urban agglomeration consisting of the provinces North Holland, South Holland, and Utrecht) an entertainment culture exists in which harddrugs (e.g.XTC, cocaine) also play a role, while in the rural areas alcohol remains the 
traditional drug of choice. In rural areas, young people often drink in private huts and sheds, where no one supervises them and a license to serve alcohol is not (yet) required (Mulder, 2005a). Students, especially fraternity and sorority students, drink a lot during the weekend, but also have many social events during the week that include alcohol (Maalsté, 2000). Young men (16-24 years) drink more than young women ( 15.5 vs. 6.9 Dutch standard drinks per week, Van Dijck \& Knibbe, 2005). In the Netherlands a standard drink contains $\sim 10$ grams of pure alcohol (vs. 13.7 grams in the United States, Centers for Disease Control and Prevention [CDC], 2006).

In general, attitude towards alcohol use by adolescents is very tolerant in the Netherlands. Many parents think it is a good idea to 'teach' children to drink alcohol, with the idea that they will be less likely to drink excessively if they are taught to drink moderately on social occasions, such as birthdays. Forty percent of Dutch parents think it is fine for 12 or 13 year old children to drink alcohol at parties with friends, without adult supervision (Van den Eijnden, 2006). Thirty percent of parents with children aged 10-15 even buy alcoholic premixed drinks (alcopops) in supermarkets especially for their offspring (Van Hoof, 2005).

The tolerant attitude towards alcohol consumption by adolescents used to be evident at school parties as well. In 2005, seventy-six percent of secondary schools served alcoholic beverages to pupils, and half of students under 16 years of age drank alcohol at these parties. As a result, one in three participants at school parties was under the influence or drunk when they went home (Mulder, 2005b). However, new research has shown that schools are becoming more aware of the importance of an alcohol-free adolescence, and the vast majority of schools no longer serve alcohol at parties on the schools' premises (National Foundation for Alcohol Prevention [STAP], 2007a).

The media, on the other hand, often portray alcohol as positive and social. Commercials for alcoholic drinks may be legally broadcast at any time during the day (however no more than $25 \%$ of the public viewing commercials may be younger than 18 years). Dutch soap series also paint a positive picture of alcohol (Hommen, 2003). As opposed to this unremitting stream of alcohol on the television, the budget for alcohol education has been cut in recent years. The budget for alcohol advertising is 273 million Euros versus 3 million Euros spent on alcohol prevention (STAP, 2003).

\subsubsection{Alcohol Policies}

A recent comparison of alcohol control policies in five domains in thirty countries ranked the Netherlands in the bottom ten (Brand, Saisana, Rynn, Pennoni, \& Lowenfels, 2007). According to the authors, the Netherlands score especially poorly on restricting alcohol availability, price policies, and alcohol advertising. Compared with other European countries only, Dutch legislation is in the middle range.

In the Netherlands, sellers are required to verify the legal age of young buyers, and are only allowed to sell alcohol when the customer is at least 16 years old (18 for spirits). Sellers are obliged to make sure that young people over 16 or 18 do not 
buy alcoholic beverages for those younger than 16 or 18 . Additionally, bartenders are required to refuse selling alcohol to someone who is intoxicated. However, there is very little enforcement of these laws (Gosselt, 2006), and servers are not liable for damages caused by actions of drunken patrons.

Since 2005, many measures to decrease youth alcohol consumption have been proposed. These include: a higher age limit for buying alcohol (18 years), stricter enforcement of age limits, making underage youth liable to punishment if they attempt to buy alcohol, a ban on sales of pre-packaged mixed drinks by supermarkets, a $€ 0.60$ (approximately $\$ 0.85$ U.S.D.) per bottle excise on "alcopops" (rum or vodka based pre-mixed beverages with $\pm 5 \%$ alcohol, that come in a variety of lemonade-like flavours and are particularly popular among teenage girls), and a ban on alcohol advertising on radio and television between 6 a.m. and 9 p.m (Ministry of Public Health, Welfare, \& Sports, 2006b; Ministry of the Interior and Kingdom Relations, 2007a). However, the propositions were either rejected by parliament or withdrawn after it became clear that parliament would not support them.

A large majority of Dutch citizens support a legal ban on sales of mixed drinks in supermarkets and a higher age limit for alcohol sales. Sixty-five percent of parents with children between the ages of 10 and 15 support removal of alcoholic mixed drinks from supermarket shelves, even though a sizeable number of them buy these mixed drinks for their children (Van Hoof, 2005). The plan to turn underage alcohol purchases into a criminal offence is also supported by a wide audience (Newcom research, 2006).

\subsection{Prevention of Excessive Alcohol Use among Youth in the Netherlands}

We have organized preventive strategies aimed at youth alcohol consumption into three categories. First, strategies that are directed at self-control are discussed. These approaches aim to decrease young people's individual inclination to drink. Next, formal social control is presented as a particular set of measures that may limit the possibility to drink excessively by means of policy and enforcement. Finally, interventions aimed at decreasing cultural acceptance of youth drinking are presented as a third category under the heading of informal social control.

\subsubsection{Self-control}

Many interventions are directed at increasing self-control, because this form of prevention meets little resistance from the alcohol industry in the Netherlands and it matches the prevailing ideology that the drinker should be held responsible for his or her drinking. An overview of these interventions in the Netherlands is listed below.

Alcohol education campaigns through mass media. Since 1986, several education campaigns aimed at excessively-drinking youth have been put forward. Although these campaigns are well-known, it has not been demonstrated that there were any 
effects on alcohol use (Van Dalen, 1999). New developments include online drinking tests for adults as well as youths (www.drinktest.nl and www.watdrinkiij.nl) and the inclusion of parents as a target group with the aim to mobilize them to increase (informal) social control over their children's drinking. The latter approach was inspired by empirical research on parental influence on youth drinking (Van der Vorst, Engels, Dekoviæ, Van Leeuwe, \& Meeus, 2005).

School-based alcohol education in secondary schools. School based alcohol and drugs education aimed at increasing skills to resist peer pressure and to increase self-control is stimulated and facilitated by the Dutch government. It is estimated that each year at least 350,000 secondary school students (ages 12 to 18) receive this kind of intervention, for which some small beneficial effects on alcohol use were reported. However, the intervention seemed to cause an increased frequency of marijuana use among those who use it (Cuijpers, Jonkers, De Weerdt, \& De Jong, 2002).

Interventions aimed at youth at increased risk for alcohol problems. There are specific interventions are available for children of alcoholics; they can get support at local group courses, self-help groups, and there exists a website (www.drankjewel.nl). An intervention by Thush et al. (2007) attempted to decrease at-risk adolescents' alcohol use by using a seven session targeted program that combined several intervention methods, such as expectancy challenge, cognitive behavioural skill training and brief motivational feedback. Additionally, biological, cognitive, and social risk factors for developing alcohol problems were discussed. Although several cognitive determinants of drinking were successfully changed, the intervention did not significantly change drinking behaviour.

Interventions in youngsters who already drink excessively. The Expectancy Challenge (Darkes \& Goldman, 1993) is an intervention that attempts to decrease excessive alcohol consumption through challenging positive alcohol expectancies (e.g. sociability, assertiveness, tension reduction). In the Netherlands, it has been applied to university students (male and female) and young men on holiday with some modest results on drinking outcomes (Wiers, Van de Luitgaarden, Van den Wildenberg, \& Smulders, 2005, Van de Luitgaarden, Wiers, Knibbe, \& Candel, 2007).

Interventions based on dual-process models. This new approach asserts that the development of addictive behaviours in adolescents is a result of an imbalance between an appetitive, approach-oriented system that becomes sensitized with repeated alcohol use and a not fully developed regulatory executlve system that is compromised by exposure to alcohol. When addictive behaviour develops and the negative consequences become more visible, the motivation to change behaviour increases. At that point however, the ability to change is compromised because the adolescent brain has changed as a result of heavy drinking (see Wiers et al., 2007). Currently, interventions are being developed that are in line with this model, such as motivational interviewing and attempts to directly modify automatic processes (Wiers et al., 2007), though outcomes data are not yet available. 


\subsubsection{Formalized control}

International research shows that formal control, such as well-enforced age limits, higher priced alcohol, restricted opening hours and a limited number of alcohol outlets can contribute to effective prevention of excessive drinking (Babor et al., 2003; Room et al., 2005). In addition, setting lower Blood Alcohol Concentration (BAC) limits may decrease frequency of driving after drinking (Wagenaar, O'Malley, \& LaFond, 2001), as well as alcohol related traffic accidents (Zwerling \& Jones, 1999).

BAC limits. As of January 2006 the BAC limit for inexperienced drivers has been lowered from $0.05 \%$ to $0.02 \%$ This adapted alcohol limit applies the first five years after the drivers license is issued for drivers who obtained their license after March 29, 2002 (Ministry of Transport and Public Works, 2005). The same BAC limit applies to moped and scooter drivers under 24 years of age. It is estimated that alcohol related traffic accidents could decrease by $5 \%$ as a result of these measures (Mathijssen, 1999).

Law enforcement. The Dutch Alcohol Licensing and Catering act is poorly enforced and as a result underage youth are able to buy alcohol in supermarkets and liquor stores (Gosselt, 2006). Even though the number of inspectors has been increased in recent years, alcohol sellers are inspected only once a year at most, causing law violations to go largely unnoticed. Another reason why the chance of catching sellers in an unlawful act is very low is that the Dutch law prohibits using underage undercover clients when inspecting alcohol sellers. In addition, only the Food and Consumer Product Safety Authority (FCPSA) is allowed to investigate violations of the law, the municipality and police have no jurisdiction to do this. Municipalities do have the authority to impose sanctions, as will be discussed in the next paragraph.

Local alcohol policy. Only a few municipalities in the Netherlands have implemented an integrated local alcohol policy. Municipalities are able to give extra priority to controlling public drunkenness (through local acts) and to withdraw a license to serve alcohol. Recently, mayors are trying to develop policies against (illegal) catering in huts and sheds. In addition, municipalities can also regulate the availability of alcohol by determining the opening hours of bars and discos. Recently, a plan was laid out to allow municipalities to raise the age limit for alcohol sales to 18 years, and to give them jurisdiction to enforce it (Ministry of the Interior and Kingdom Relations, 2007b).

Alcohol Prices. Although excise tax is charged on alcohol, the effects in the Netherlands have been modest, because a higher tax has not increased the price of alcohol sufficiently. Due to sideline jobs and higher allowances, the average income of adolescents has increased much more than the price of alcohol (Interview / NSS, 1999). Alcohol prices in the Netherlands are about average for Europe (Statistics Netherlands [CBS], 2004), but beer prices are relatively low (WHO, 2004).

Availability restrictions. Alcoholic beverages with less than $15 \%$ alcohol are sold in supermarkets, tobacco shops, and liquor stores. Spirits may only be sold in liquor stores, however pre-packaged mixed drinks are sold in supermarkets as well.

Restrictions on alcohol commercials. Restrictions on alcohol commercials could be a useful measure, however, the alcohol industry is allowed to regulate itself by 
adhering to the "Advertising Code for Alcoholic Beverages" that they developed. The code is highly criticized because 1) alcohol advertising still reaches too many young people, 2) the code is regularly violated, and 3) several articles are ambiguous. If the code is violated, the Advertising Code Committee only makes recommendations, no sanctions ensue and the judgment only comes after the advertising campaign has already ended (STAP, 2007b).

\subsubsection{Informal social control}

In the Netherlands, informal social control on adolescents' alcohol use is limited or absent because adolescent drinking is widely accepted.

Family. Parents' drinking norms influence their children's alcohol use (Spijkerman, Van den Eijnden, Overbeek, \& Engels, 2007). Research has indicated that an early onset of drinking predicts alcohol-related problems later in life (Grant \& Dawson, 1997). Therefore, it is important to educate parents on raising their children to be non-drinkers until they are at least 16 years old (Van der Vorst et al., 2005). In 2007, a national campaign has been launched that focuses on parents of adolescents.

Community projects. Attention to community projects is increasing; however, at the moment only a few community projects aimed at prevention of alcohol problems in youth are implemented. Health problems caused by excessive drinking in youth are often not the primary concern of local actors organising a community project. Instead, they are often most concerned with public nuisance, which can be solved by measures that do not decrease alcohol consumption such as increasing the availability of night buses and cabs plus faster police intervention in the case of public disturbances.

\subsubsection{Translation of Alcohol Prevention}

Many prevention methods in the Netherlands were translated from basic research, such as alcohol education campaigns and school-based alcohol prevention that were inspired by Ajzen's Theory of Planned Behavior (1991) Other interventions were translated from protocols that were developed in other countries, such as the Expectancy Challenge (Darkes \& Goldman, 1993) which was translated into Dutch and adapted several times (e.g. shortened and made suitable for use with young women). Finally the program was taken from the "surrogate bar" (a room at the university that was decorated as a café) into actual drinking contexts of young people on holiday (Van de Luitgaarden et al., 2006). Other Dutch prevention practices were also translated from research elsewhere in the world, such as the decision to lower the BAC limit for inexperienced drivers and the idea to organize community projects. Although internationally inspired preventive practices have been translated into Dutch prevention practice, many important international successes have not yet been adopted. This is particularly the case for higher age limits, higher prlced alcohol, restrictions on alcohol advertising, and better law enforcement, although these continue to be on the political agenda. 


\subsection{Discussion}

In the Netherlands, alcohol is still readily available to almost anyone who attempts to purchase it. This is caused in large part by the lack of enforcement of age laws, the idea that (young) people are responsible for their own alcohol use, the tolerant mentality regarding adolescents' alcohol use, and the strong position of the alcohol lobby. Implementing effective alcohol policies in a society that is tolerant of youth drinking and that emphasises personal choice is a daunting challenge.

Fortunately, in the past few years many professionals and politicians are starting to recognize that alcohol education aimed at increasing self-control has not had any effect on alcohol use the past decennia; hence, there is a need for more formal preventive strategies. A culture change is needed and media attention and education campaigns are particularly suited for agenda setting and for promoting acceptance of new measures. The public opinion is already shifting towards more restrictive formal measures (Newcom Research, 2006). New measures could include restrictions on the number of alcohol outlets, a ban on alcohol advertising, higher taxes on alcoholic drinks, limiting opening hours of bars and, especially, enforcement of existing laws.

Based on international research, a higher age limit for buying mildly alcoholic drinks (18 instead of 16) would be an effective measure to decrease adolescent drinking (Wagenaar \& Toomey, 2002). It would be more uniform and more clear-cut to both youth and parents, and therefore potentially easier to enforce than the current dual-age system. In addition, it would give support to parents setting strict rules on alcohol use. However, in order to overcome resistance by alcohol sellers and buyers, a larger number of inspectors of the Alcohol Licensing and Catering Act are needed to enforce such a law effectively. More funding is necessary for adequate enforcement of the Alcohol Licensing and Catering Act, and enforcement needs to be co-ordinated with municipalities and their local policies. Compliance with alcohol laws can be increased if the police are given jurisdiction to enforce alcohol laws. A proposal to achieve this is currently under consideration. The current Dutch situation can be described as one of missed opportunities, but also as one awaiting new developments. Time will tell which measures will actually come into effect, but our guess is that the next five years will bring about evidence-based policy changes. 


\section{References}

Ajzen, I. (1991). The theory of planned behavior. Organizational Behavior and Human Decision Processes 50, 179-211.

Babor, T., Caetano, F., Casswell, S., Edwards, G., Giesbrecht, N., Graham, K. et al., (2003) Alcohol: No ordinary commodity-A consumer's guide to public policy. Oxford, U.K.: Oxford University Press.

Brand, D.A., Saisana, M., Rynn, L.A., Pennoni, F., \& Lowenfels, A.B. (2007). Comparative analysis of alcohol control policies in 30 countries. PLoS Medicine, 4(4): e151. Retrieved June 7, 2007, from http://www.medscape.com/viewarticle/556169.

Centers for Disease Control and Prevention (2006). Alcohol and public health. Frequently asked questions. Retrieved October 10, 2007, from http://www.cdc.gov/alcohol/faqs.htm\#4

Cuijpers, P., Jonkers, R., De Weerdt, I., \& De Jong, A. (2002). The effects of drug abuse prevention at school: The 'Healthy School and Drugs' project. Addiction, 97, 67-73.

Dahl, R.E., Spear, L.P., editors (2004). Adolescent brain development: Vulnerabilities and opportunities. Annals of the New York Academy of Sciences, vol. 1021.

Darkes, J. \& Goldman, M.S. (1993). Expectancy challenge and drinking reduction: experimental evidence for a mediational process. Journal of Consulting and Clinical Psychology, 61, 344-353.

Engels, R.C.M.E., \& Knibbe R.A. (1999) Young people's alcohol consumption from a European perspective: risks and benefits. European Journal of Clinical Nutrition, 54, 52-55.

Gosselt, J.F. (2006). Drank kopen kent geen leeftjj,. A/coholverkoop aan jongeren onderde wettelijk toegestane leeftijdsgrens: een onderzoeksprotocol en een studie naar de naleving van de Nederlandse Drank-en Horecawet [Buying alcohol is ageless. Alcohol sales to underage youth: a research protocol and a study of compliance with the Dutch Alcohol Licensing and Catering act]. Utrecht: STAP.

Grant, B.F., \& Dawson, D.A. (1997). Age at onset of alcohol use and its association with DSM-IV alcohol abuse and dependence: results from the national longitudinal alcohol epidemiologic survey. Journal of Substance Abuse, 9, 103-110.

Heath, D.B. (1995) An anthropological view of alcohol and culture in international perspective. In International Handbook on Alcohol and Culture. pp. 328-347. London: Greenwood

Hibell, B., Andersson, B., Bjarnason, T., Ahlström, S., Balakireva, O., Kokkevi, A., et al. (2004). The ESPAD report 2003; Alcolol and other drug use ameng students in 35 European countries. Stockholm: The Swedish Council for Information on Alcohol and Other Drugs (CAN) and the Pompidou Group at the Councll of Europe.

Hofstede, G. (1991), Cultures and organization: Software of the mind. Glasgow, UK: McGraw Hill International.

Hommen, K. (2003). Verborgen alcoholreclame. Een onderzoek naar de weergave van alcohol op de Nederlandse televisie [Hidden alcohol advertising. A study of the image of alcohol on Dutch television]. Utrecht/Amsterdam: STAP/University of Amsterdam.

Interview/ N5S (1999). Jongeren'g9 [Youth '99]. Amsterdam: Author.

Knibbe, R.A., Joosten, J., Choquet, M., Derickx, M., Morin, D., \& Monshouwer, K. (2007). Culture as an explanation for substance-related problems: a cross-national study among French and Dutch adolescents. Social Science and Medicine, 64 (3), 604-616.

Maalsté, N. (2000). Ad Funduml Een blik in de gevarieerde drinkcultuur van het Nederlandse studentenleven [Ad Funduml A look into the varied drinking culture of Dutch students]. Utrecht: CVO.

Mathljssen, M.P.M. (1999). Schatting van de effecten van verlaging van de wettelljke limiet voor alcoholgebrulk in het verkeer; Advies aan het Ministerie van Verkeer en Waterstaat lAn estimate of the effects of lowering the legal alcohol limit for taking part in traffic; Advice to the Ministry of Transport, Public Works and Water Management]. Leidschendam: SWOV.

Minlstry of Public Health, Welfare, \& Sports (2006a). Preventlenota Klezen voorGezond Leven (Government amendment 'Choosing a healthy [ife'].(Parliamentary document No. PG/OGZ/2.720,104). Retrieved June 9, 2007, from http://www.minvws.nl/images/preventienota_tcm19-137692.pdf 
Ministry of Public Health, Welfare, \& Sports (2006b). Voorgenomen wetgeving alcohol en tabak. IProposed legislation on alcohol and tobaccol. (Parliamentary document No. VGP/ADT 2.684.379). Retrieved June 9, 2007, from http://www.minvws.nl/kamerstukken/vgp/2006/voorgenomen-wetgevingalcohol-en-tabak.asp

Ministry of the Interior and Kingdom Relations (May 5, 2007a). Rijk en gemeenten in IJsselland pakken alcoholmisbruik jongeren aan lGovernment and communities in Usselland tackle alcohol abuse by youth]. Press release. Retrieved June 7, 2007, from http://www.minbzk.nl/actueel/nieuws-en?Actltmldt=105613

Ministry of the Interior and Kingdom Relations (June 1, 2007b). Gemeenten bes/issen over leeftijdsgrens alcoholverkoop [Municipalities decide on age limit alcohol sales]. Press release. Retrleved June 7 , 2007, from http://www.minbzk.nl/actueel/nieuws--en?Actltmldt=106035

Ministry of Transport and Public Works (2005). Wijziging van de Wegenverkeerswet 1994 in verband met verlaging van de wettelijke alcohollimiet voor beginnende bestuurders [Amendment of the 1994 traffic law concerning the lowering of the legal alcohol limit for inexperienced drivers]. (Parliamentary document No. HDJZ/AWW/2005-348). Retrieved June 7, 2007, from http://www.verkeerenwaterstaat.n!

Monshouwer, K., Dorsselaer, S. Van, Gorter, A., Verdurmen, J, et al. (2004). Jeugd en riskant gedrag. Kerngegevens uit het peilstationsonderzoek 2003. Roken, drinken, drugsgebruik en gokken onder scholieren vanaf tien jaar [Youth and risky behavior. Core data 2003. 5moking, drinking, drug use, and gambling in pupils over 10 years old]. Utrecht: Trimbos-institute.

Mulder, J. (2005a). indrinken op eigen erf [Pre-drinking on own property]. Utrecht: STAP.

Mulder, J. (2005b). Drinken op school. Landelijk scholierenonderzoek naar de alcoholverstrekking en het alcoholgebruik binnen het voortgezet onderwijs en op schoolfeesten [Drinking in school. National survey of alcohol sales and alcohol use in secondary schools and on school parties]. Utrecht: STAP.

National Foundation for Alcohol Prevention (2003). Factsheet investeringen in alcoholreclame [Factsheet 'investments in alcohol advertising']. Utrecht: Author.

National Foundation for Alcohol Prevention (October 4, 2007a). Alcoholvrije scholen in opkomst [Alcoholfree schools on the rise]. Press release. Retrieved October 15, 2007, from http://www. alcoholpreventie.nl/persbericht/

National Foundation for Alcohol Prevention (2007b). Zelfregulering beschermt jongeren niet tegen invloed van alcoholreclame. Samenvattende beschrijving van beschikbare bronnen over het functioneren van de zelfregulering van alcoholreclame in Nederland [Self regulation does not protect youth from the influence of alcohol advertising. Concluding description of available sources about the functioning of self-regulation of aicohol advertising in the Netherlands]. Utrecht: Author.

National Institute on Alcohol Abuse and Alcoholism (2005). The effects of alcohol on physiological processes and biological development. Alcohol Research and Health, 28(3), 125-31.

Newcomb, M.D., \& Bentler, P.M. (1989). Substance use and abuse among children and teenagers. American Psychologist, 44, 242-248.

Newcom Research \& Consultancy (2006). Meerderheid Nederlanders voor strakkere regelgeving alcohol [A majority of Dutch people are in favor of stricter legislation on alcohol]. Retrieved June 9, 2007 from https://secure.mijnopinie.nl/index.php?pagina_id=2\&nieuws_id=32

Room, R., Babor, T., and Rehm, J. (2005). Alcohol and public health. Lancet, 365, 519-530.

Spijkerman, R., Van den Eijnden, R.J.J.M., Overbeek, G., \& Engels, R.C.M.E.(2007). The impact of peer and parental norms and behavior on adolescent drinking: The role of drinker prototypes. Psychology \& Health, 22(1), 7-29.

Statistics Netherlands (2004). Nederland niet duur, niet goedkoop [The Netherlands; not expensive, not cheap]. Retrieved June 7, 2007, from http://www.cbs.nl/nl-nl/menu/themas/prijzen/publicaties/artikelen/archief/2004/2004-1623-wm.htm

Thush, C., Wiers, R.W., Theunissen, N., Van den Bosch, J., Opdenacker, J., Van Empelen, P. et al. (2007). A randomized clinical trial of a targeted intervention to moderate alcohol use and alcohol-related problems in at-risk adolescents. Pharmacology, Biochemistry and Behavior, 86, 368-376. 
Trimbos Institute (2007). Nationale drug manitor. Jaarbericht 2006 [National drug monitoring system. Annual report 2006]. Utrecht: Author.

Van Amerongen, R. (1988). Geschiedenis van de alcoholpreventie [History of alcohol prevention]. In J.C. van der Stel \& W.R. Buisman (Eds.), Alcoholpreventie: achtergronden, praktijk en beleid (pp. 30-52). Samsom press: Alphen aan den Rijn / Brussels.

Van Dalen, W.E. (1999). "Drank maakt meer kapot dan je lief is". Een beschrijving van het 12,5 jaar bestaan van een unieke campagne ["Booze ruins more than you care for". A description of the 12.5 year anniversary of a unique campaign]. In: Handboek vers/aving (pp. C2050-1 - C2050-21). Utrecht: Trimbos institute.

Van de Luitgaarden, J., Wiers, R.W., Knibbe, R.A. and Boon, B.J. (2006). From the laboratory to real life: A pilot study of an expectancy challenge with 'heavy drinking' young people on holiday. Substance Use and Misuse, 41, 353-368.

Van de Luitgaarden, J., Wiers, R.W., Knibbe, R.A., \& Candel, M.J.J.M. (2007). single-session Expectancy Challenge with young heavy drinkers on holiday. Addictive Behaviors, 32, 2865-2878.

Van den Eijnden, R.J.J.M. (2006, January). Alcoholgebruik jongeren 12-17 jaar: De rol van de ouders [Alcohol use among young people between the ages of 12-17: The role of parents]. Presentation at the vearly National Alcohol conference, Rotterdam, the Netherlands.

Van der Vorst, H., Engels, R.C.M.E., Dekovize, M., Van Leeuwe, J., Meeus, W.H.E. (2005). The role of alcohol specific socialization on adolescents' drinking behavior. Addiction, 100, 1464-1474.

Van Dijck, D. \& Knibbe, R.A. (2005). De prevalentie van probleemdrinken in Nederland IThe prevalence of problem drinking in The Netherlands]. Maastricht: UM.

Van Hoof, J.(2005). Mixdrankjes uit de supermarkt? Onderzoek naar het koopgedrag van ouders van kinderen van 10 tot en met 15 jaar in relatie tot alcohol en mixdrankjes in het bijzonder IMixed drinks banned from supermarket shelves? A study of parents of children aged 10-15 and their buying behavior of mixed drinks and other alcoholic drinks]. Twente/Utrecht: University of Twente / STAP.

Van Kleef, L.E. \& Van der Lely, N. (2006). Jongeren met alcoholintoxicatie: een groeiend probleem [Alcohol intoxication in young people: a growing problem]. Nederlands tijdschrift voor Geneeskunde, 150(46), 2521-2522.

Wagenaar, A. C., O'Malley, P. M., \& LaFond, C. (2001). Lowered legal blood alcohol limits for young drivers: Effects on drinking, driving, and driving-after-drinking behaviors in 30 states. American Journal of Public Health, 91, 801-804.

Wagenaar, A.C. \& Toomey, T.L. (2002). Effect of minimum drinking age laws: review and analyses of the literature from 1960-2000. Journal of Studies on Alcohol, Supp 114, 206-225.

Wiers, R.W., Bartholow, B.D., van den Wildenberg E., Thush, C., Engels, R.C., Sher, K.J. et al, (2007). Automatic and controlled processes and the development of addictive behaviors in adolescent: $A$ review and a model. Pharmacology, Biochemistry and Behavior, 86, 263-283.

Wiers, R.W., Hoogeveen, K.J. Sergeant, J.A., \& Gunning, B.W. (1997). High- and low-dose alcohol-related expectancies and the differential associations with drinking in male and female adolescents and young adults. Addiction, 92, 871-888.

Wiers, R.W., Van de Luitgaarden, J., Van den Wildenberg, E., \& Smulders, F.T.Y.(2005). Challenging implicit and explicit alcohol-related cognitions in young heavy drinkers. Addiction, 100, 806-819.

Wiers, R.W., Van Woerden, N., Smulders, F.T., \& De Jong, P.J. (2002). Implicit and explicit alcohol-related cognitions in heavy and light drinkers, Journal of Abnormal Psychology, 111(4), 648-658.

World Health Organization (2004). Global Status Report: Alcohol Pollcy. Geneva: Author.

Zwerling, C., \& Jones, M. P. (1999). Evaluation of the effectiveness of low blood alcohol concentration laws for younger drivers. American Journal of Preventive Medicine, 16(1S), 76-80. 
CHAPTER 3

\section{Challenging implicit and explicit alcohollrelated cognitions in young heavy drinkers}




\begin{abstract}
This study aimed to test whether an Expectancy Challenge (EC) changes implicit and explicit alcohol-related cognitions and binge drinking in young heavy drinkers. This is important for theoretical and practical reasons: the EC presents a critical test for the hypothesized mediational role of alcohol cognitions and the EC has been presented as one of the promising interventions to counter alcohol problems in heavy drinking youth. Ninety-two heavy drinking college and university students (half women) were randomly assigned to the EC or control-condition (a sham alcoholexperiment in the same bar-lab). Explicit alcohol cognitions and alcohol use were assessed with paper and pencil measures. Alcohol use was assessed prior to the experiment and during a one month follow-up. Implicit alcohol-related cognitions were assessed with two versions of the Implicit Association Test (IAT), adapted for alcohol that assess implicit valence and implicit arousal associations with alcohol. The EC resulted in decreased explicit positive arousal expectancies in men and women alike. There was some evidence for a differential reduction in implicit arousal associations, but findings depended on the version of the IAT and on the scoringalgorithm used. In men but not in women there was a short-lived reduction in prospective alcohol use (significant in week 3 of the follow-up), and this reduction was partially mediated by the decrease in explicit positive arousal expectancies. These findings suggest that an EC successfully changes explicit alcohol cognitions and that this may have short-lived beneficial effects in heavy drinking young men.
\end{abstract}




\subsection{Introduction}

Recently, there has been great concern about college drinking. In the United States, each year 1400 students die, and 500000 are injured under the influence of alcohol (Hingson et al., 2002). In the Netherlands, where this study took place, alcoholrelated disorders are the most prevalent psychiatric diagnosis in young men (Bij), Ravelli \& van Zessen, 1998), and students drink more than their peers (Maalsté, 2000).

Prevention efforts can be aimed at the individual or at the environment. Among the individual-focused strategies, cognitive-behavioural and motivational interventions have shown greater efficacy than educational interventions (Larimer \& Cronce, 2002). The same review indicated that the "Expectancy Challenge" (EC), is a promising cognitive behavioural intervention for college-age men. These expectancies are hypothesized to mediate the relationship between distal risk factors (that are difficult to change) and drinking outcomes (Goldman, 1999). Therefore, a successful challenge of positive expectancies should result in reduced drinking (Darkes \& Goldman, 1993, 1998). In line with this idea, Darkes and Goldman (1993; 1998) presented evidence for reductions in both positive expectancies and in short-terms drinking. However, 'mediation was not demonstrated in any of these studies' (Jones, Corbin \& Fromme, 2001b, p. 1673). Mediation will be tested formally here for the first time.

Despite some controversy regarding the evidence supporting the EC, there is agreement that a number of theoretical and practical issues should be addressed (Del Boca \& Darkes, 2001; Jones et al., 2001a,b; Wiers, 2002a; Wiers et al., 2003). One important theoretical issue concerns the nature and measurement of expectancies. Traditionally this has been done with questionnaires. Recently there has been a growing interest in more implicit assessments of cognitive variables in alcohol and addiction-research (e.g., Stacy, 1997; Ames \& Stacy, 1998; Wiers, et al., 2002; Wiers \& Stacy, 2006). Implicit associations have been defined as introspectively unidentified (or inaccurately identified) traces of past experience that mediate favorable or unfavorable feeling, thought, or action' (Greenwald \& Banaji, 1995, p.8). Implicit assessments are carried out for various reasons: first, to prevent socialdesirable responding (e.g. Greenwald, McGhee, \& Schwartz, 1998; Fazio \& Olson, 2003). This may be relevant here, because the message of the EC is clear: positive expectancies are largely incorrect (i.e. they are placebo effects) and one could benefit from drinking less. Hence, a tendency to respond in a socially desirable way could affect both self-reported expectancies and self-reported drinking and create a spurious 'treatment effect'. A second reason to include implicit measures is that they could tap different underlying cognitive motivational processes (e.g. Stacy, 1997; Wiers et al., 2002; Palfai \& Ostafin, 2003). Neurobiological research demonstrated that subcortical circuits involved in emotion and motivation are important in addiction and these circuits are not directly accessible for introspection (White, 1996; Bechara, Damasio, \& Damasio, 2003; Robinson \& Berridge, 2003). Implicit measures have been shown to correlate highly with activation of these structures in functional Magnetic Resonance Imaging (f-MRI) studies (e.g. Phelps et al., 2001) and very brief- 
IV presented pictures activate these structures in the absence of awareness (Cunningham et al., 2004). Further, several studies have found that implicit and explicit alcohol-related cognitions predict unique variance in alcohol use (Stacy, 1997; Wiers et al., 2002; Kramer \& Goldman, 2003), and interventions may differentially affect implicit and explicit cognitions (e.g. Teachman \& Woody, 2003). Hence, for both reasons it is desirable to assess implicit and explicit alcohol-related cognitions in an EC. This has not been conducted previously.

Here, implicit alcohol associations were assessed with two versions of the widely used Implicit Association Test (IAT, Greenwald et al., 1998): one assessing alcoholvalence associations (ar implicit attitudes) and one assessing alcohol-arousal associations (arousal versus sedation, Wiers et al., 2002). With these two IATs, we found in previous research that heavy drinkers hold implicit alcohol-arousal associations which was not the case for light drinkers, and that both heavy and light drinkers hold negative implicit alcohol associations (both compared with sodas, Wiers et al., 2002). Recently, it has been found that alcoholics in treatment, like heavy drinkers, hold implicit negative and arousal associations with alcohol (De Houwer et al., 2004). Here, arousal and sedation expectancies and attitudes were also assessed with explicit tests, using the same words. We hypothesized that the EC would reduce explicit arousal expectancies and increase explicit sedation expectancies (cf. Dunn, Lau, \& Cruz, 2000). We hypothesized further that if a change would occur in implicit associations, this would be on the arousal-IAT (since this dimension differentiated heavy from light drinkers, Goldman et al., 1999; Wiers et al., 2002). Recent research in social cognition has indicated that implicit associations may be more malleable than thought previously (for reviews, see Blair, 2002; Wiers et al., 2004). Implicit and explicit assessments of valence were included because a differential change in the EC group in arousal would be more convincing in the absence of a change in valence (discriminant validity). Secondly, these two basic dimensions have been found to underlie both emotions and expectancies (Goldman et al., 1999).

Another theoretical issue is how to treat the control-group. Control-groups in previous EC studies involved assessment-only groups or in one case an informationonly contrast group (Darkes \& Goldman, 1993). Both types of control conditions took place in neutral rooms, which leaves the possibility open of an a-specific effect on alcohol-related cognitions of drinking alcohol in a group of youth in a barlaboratory, because both implicit and explicit measures of drug-related cognitions have been shown to be sensitive to context-effects (Wall et al., 2001; Sherman et al., 2003). For optimal comparability with respect to context, participants in our control-group drank the same alcoholic or placebo drinks in the same bar-laboratory as the EC group. The crucial difference between conditions was the absence of placebo manipulation and expectancy information.

In addition to these theoretical issues, the present study addressed two more practical issues. The first concerns the nature of the EC. Negative replications cited by Jones et al. (2001a) were either information-only ECs or constituted of a single experiential session (Wiers, 2002a). Here we tested an extended single-session EC, that combined contents of both experiential sessions of the original protocol into 
one session, followed by homework to promote cognitive elaboration. The second issue concerned gender: previous EC studies included either only young men (Darkes \& Goldman 1993; 1998), or found no effect for women (Dunn, et al., 2000; Musher-Eizenman, \& Kulick, 2003). In a recent study, we adapted the second session on sex-expectancies to be more suitable for women and found a significant decrease in positive arousal expectancies in women (Wiers \& Kummeling, 2004). This adapted version was used here.

To summarize, the present study investigated the effects of a single session extended experiential EC on implicit and explicit alcohol-related cognitions and on alcohol use during a 1-month follow-up. We hypothesized that the EC would reduce arousal expectancies (explicit and implicit) and alcohol use. In case both occurred, a formal test of mediation was performed.

\subsection{Method}

\subsubsection{Participants}

Initial recruitment asked students to participate in Maastricht University and vocational colleges close by. Students were asked to participate in a 'fun experiment on the effects of alcohol'. Interested students were administered a brief telephoneinterview, asking them their age, gender and weekly alcohol use (asked per average day). Inclusion criteria were: 15 to 50 European standard-drinks per week for men and 13 to 45 for women (similar to Darkes \& Goldman, 1993: European standarddrinks contain about $10 \mathrm{~g}$ pure alcohol compared with $14 \mathrm{~g}$ in the United States; however a somewhat heavier drinking sample was recruited here). A randomization scheme (stratified for gender) was used to assign eligible participants to conditions.

A total of 96 undergraduate students ( 48 women) were scheduled to participate. Four failed to show up, leading to a final sample of 92 participants (46 women), with mean age of 20.5 years. Eleven participants self-identified with another nationality than 'Dutch', all of whom had passed a test demonstrating that they were fluent in Dutch. Average alcohol consumption per week was 29.5 standard-drinks (range: 10-70; mean 34 for men and 24 for women per week). Participants binged on 4.3 occasions during the past two weeks (range: $0-12$ ). On the Rutgers Alcohol Problems Index (RAPI, White \& Labouvie, 1989; 2000), participants scored 14.3 (range: 2-37); just below the average of clinical samples (White \& Labouvie, 1989). On the Alcohol Use Disorder Identification Test (AUDIT, Saunders et al., 1993) participants scored an average of 11.7 (range: $5-26$ ). Here $74 \%$ scored 10 or higher (cutoff for alcohol problems, Saunders, et al, 1993), and 90\% scored 8 or higher (hazardous drinking, Palfai \& Ostafin, 2003). Men scored significantly higher on all alcohol variables $(p<.01)$, except the RAPI $(p>.25)$. Interestingly, only one participant indicated he had an alcohol problem himself, when asked in the context of a family tree. Hence, the sample can be described as hazardous or problem drinkers, who do not think they have a drinking problem themselves. For this case, the indirect re- 
cruitment (focus on alcohol use, not on problems) and the EC, which does not directly target alcohol problems, was judged ethically appropriate.

\subsubsection{Materials and Measures}

Alcohol Use. Alcohol use was measured with a self-report questionnaire (Wiers et al., 1997) based on the time-line follow back method, which has been found to be reliable and valid (Sobell \& Sobell, 1990). With this questionnaire, estimates for drinking prior to the experiment were generated (Wiers et al., 1997). After the intervention, participants kept an alcohol-diary, which they handed in after 1 month (there was no check on actual daily recording). From these measures, a quantityfrequency index (drinks per week) and number of binges were calculated.

Alcohol-Related Problems. The RAPI (White \& Labouvie, 1989) was used, which measures social and health-related problems adolescents and young adults experienced with alcohol. We used the 18-items version, which correlates .99 with the 23-items version (White $\&$ Labouvie, 2000). Internal consistency (Cronbach's $\alpha$ ) was 0.81 . Further, the Alcohol Use Disorders Identification Test (AUDIT) (Saunders, et al., 1993) was used ( $\alpha=0.66)$.

Implicit Alcohol Associations. Two adapted versions of the IAT (Greenwald, et al., 1998) were presented in balanced order, one measuring the positive-negative dimension (Valence-IAT) and one the arousal-sedation dimension (Arousal-IAT), both described in detail in Wiers et al. (2002). The IAT is a computerized categorization task that offers a method to assess indirectly the relative strength of associations between concepts. Participants sort stimuli into four concepts, using two response buttons, in two combinations. Two concepts are the 'targets' (here: 'alcoholic drinks' vs. 'sodas'), and two are the attributes (Valence-IAT: 'positive' vs. 'negative'; Arousal-IAT: 'active' vs. 'passive'). The IAT-effect is the difference in reaction times for the two combinations of targets and attributes (see Table 1).

The target-words for both IATs were for the alcoholic drinks: beer, wine, port, whisky, vodka, and rum; for Sodas: coke, 'cassis', 'sinas' (lemonades), 'spa' (sodawater), tonic, and juice. In the valence-IAT, positive words were: sociable, good, pleasant, nice, enjoyable, sympathetic; negative words: antisocial, bad, unpleasant, stupid, obnoxious, tedious. For the arousal-IAT, the arousal words were: energetic, lively, funny, cheerful, smart, aroused; sedation words: relaxed, sleepy, woozy, quiet, calm, and listless. Words were based on previous research using multidimensional scaling (Goldman et al., 1999) and on first associates in Dutch students (Wiers, 2002b). The Dutch words were matched for prevalence and number of syllables.

Because the IAT-effect is a difference score between two different pairings of stimuli, it can be assessed in two different orders. The IAT-effect is larger when the association that generates the fastest responses comes first (the 'compatible' or CR order) than in the reverse combination (RC order, Greenwald et al., 1998; Wiers et al., 2002). Order-effects were controlled both as within and between-subject factors, generating two IAT-effects per IAT, a CR-IAT and an RC-IAT (Table 1; cf. Greenwald et al., 1998; Wiers et al., 2002). 
Table 1: Sequence of blocks in the arousal IAT (CRRC type)

\begin{tabular}{|c|c|c|c|c|}
\hline Phase & Block & Label & $\begin{array}{l}\text { Words assigned } \\
\text { to one key }\end{array}$ & $\begin{array}{l}\text { Words assigned } \\
\text { to the other key }\end{array}$ \\
\hline \multirow[t]{2}{*}{ Practice Target } & 1 & Target & Alcohol & Soda \\
\hline & 2 & Target & Alcohol & Soda \\
\hline \multirow[t]{2}{*}{ Practice Attribute } & 3 & Attribute & Arousal & Sedation \\
\hline & 4 & Attribute & Arousal & Sedation \\
\hline \multirow[t]{3}{*}{ Compatible "C" } & 5 & Comp. Practice & Alcohol + Arousal & Alcohol + Sedation \\
\hline & 6 & Comp. Test 1 & Alcohol + Arousal & Alcohol + Sedation \\
\hline & 7 & Comp. Test 2 & Alcohol + Arousal & Alcohol + Sedation \\
\hline PracticeReverse Target & 8 & Reverse target & Soda & Alcohol \\
\hline \multirow[t]{4}{*}{ Reversed " $R$ " } & 9 & Reversed Practice & Alcohol + Sedation & Alcohol + Arousal \\
\hline & 10 & Reversed Test 1 & Alcohol + Sedation & Alcohol + Arousal \\
\hline & 11 & Reversed Test 2 & Alcohol + Sedation & Alcohol + Arousal \\
\hline & 12 & $R$ target & Soda & Alcohol \\
\hline \multirow[t]{4}{*}{ Reversed "R" } & 13 & Reversed Practice & Alcohol + Sedation & Alcohol + Arousal \\
\hline & 14 & Reversed Test 1 & Alcohol + Sedation & Alcohol + Arousal \\
\hline & 15 & Reversed Test 2 & Alcohol + Sedation & Alcohol + Arousal \\
\hline & 16 & Target & Alcohol & Soda \\
\hline \multirow[t]{3}{*}{ Compatible " $C$ " } & 17 & Comp. Practice & Alcohol + Arousal & Alcohol + Sedation \\
\hline & 18 & Comp. Test 1 & Alcohol + Arousal & Alcohol + Sedation \\
\hline & 19 & Comp. Test 2 & Alcohol + Arousal & Alcohol + Sedation \\
\hline
\end{tabular}

Note: Half the participants received the phases in the opposite order (reversed, compatible, compatlble, reversed: RC-CR). The other IAT, was the valence-IAT.

Recently, Greenwald, Nosek and Banaji (2003) proposed a new scoring-algorithm for the IAT-effect, in which practice-blocks are included and results are standardized at the level of the participant. We report the IAT-effects for the new 'D-600' algorithm and tabulate the results for the original scoring algorithm (difference in RT between the two combination test-phases), for ease of interpretation and comparability with earlier work. In the case of different results found for the original algorithm, these are reported.

The psychometric properties of the IAT have generally been described as good (Greenwald \& Nosek, 2001). The internal consistency (calculated as in Greenwald et al., 2003) was 0.65, for the Valence-IAT and 0.68 for the Arousal-IAT 0.68. Testretest reliabilities were 0.73 and 0.75 , respectively (controls only, whole sample, see Table 2). These values are much higher than for other measures of implicit associations (cf. Bosson, Swann \& Pennebaker, 2000).

Explicit Alcohol-Related Cognitions. The attribute words used in the two IATs were used to construct explicit equivalents of the valence and arousal dimension (as in Wiers et al., 2002). The explicit measure of valence (global attitudes) consisted of six semantic differentials (e.g. 'drinking alcohol is good -...- bad') with the Visual Analogue Scale (VAS). In line with expectancy-research, unipolar VAS-scales were 


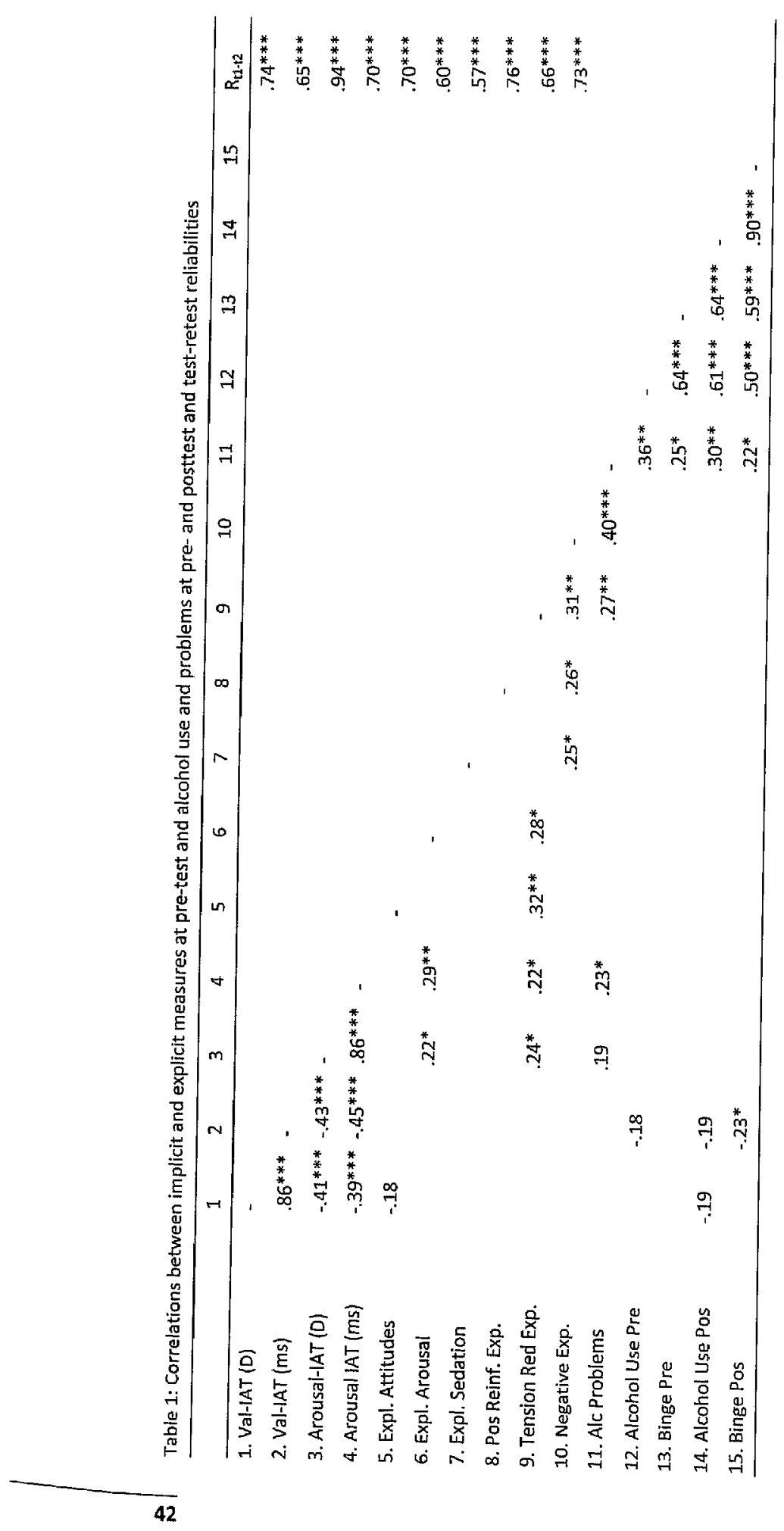


used to assess explicit arousal and sedation expectancies. Internal consistencies were: VAS-attitudes 0.81; VAS-arousal 0.86; VAS-sedation 0.75 . In addition, participants filled out a longer questionnaire with items briefly describing situations that assessed positive and negative expectancies for low and high doses of alcohol (an updated version of Wiers et al., 1997). Three global scales were analysed: positive reinforcement expectancies $(\alpha=0.87$ ), negative reinforcement expectancies $(\alpha=$ $0.76)$, and negative expectancies $(\alpha=0.76)$.

Non-specific effects. The Subject Reaction Questionnaire (SRQ, Darkes \& Goldman, 1993) assessed the intervention's credibility and acceptability (1-5 Likertscales).

\subsubsection{Procedure}

Pre-test. One week before the intervention, participants came to the laboratory and performed the two IATs, followed by questionnaires (implicit before explicit assessment, cf. Bosson et al., 2000).

Intervention. The intervention took place in our bar-laboratory. Participants signed an informed consent, in which they agreed to drink two drinks containing alcohol (placebos contained a minimal dose of alcohol on the rim of the glass, as advised by Marlatt \& Rohsenow, 1980). All participants signed the same ethicaliy approved informed consent. They then completed questionnaires assessing background variables. The rest of the session depended on experimental condition. $E C$. The procedure followed closely the original protocol of the two experiential sessions by Darkes and Goldman (1993), with three differences: first, during the first drinking session, all participants were told they were drinking vodka-tonic (active deception, Marlatt \& Rohsenow, 1980). Secondly, these sessions were not separated by a week but by a short break. Thirdly, the second session targeting sexual expectancies was adapted for use in mixed gender groups (Wiers \& Kummeling, 2004).

Details concerning the EC can be found in Darkes and Goldman (1993) and in Wiers and Kummeling (2004) or are available upon request. The challenge ended with a homework assignment: participants were asked to write a short essay on expectancies in the media and in their own life and to keep an alcohol diary. Before leaving, the SRQ was completed and breath alcohol level was measured. Participants signed for the alcohol-level and were instructed to be extra careful. No participant left above the legal limit ( 0.5 per mil).

Control-group: Sham Alcohol Experiment. The control group was openly split in two: half the participants received vodka and tonic and half tonic only. After beverage consumption, participants performed neuropsychological tests. No information on expectancies followed. The session ended as in the EC: homework instruction (alcohol-diary), SRQ, and breath alcohol level were measured.

Post-test. The post-test took place 1 week after the intervention and was identical to the pre-test.

One Month Follow-up. One month after the post-test, participants handed in their alcohol diaries and received a monetary reward. 


\subsection{Results}

\subsubsection{Success of Randomization}

There were no differences in any of the alcohol-related or background variables between the two experimental conditions or in the interactions between condition and gender (all $p^{\prime} s>0.25$ ).

\subsubsection{Implicit Alcohol Associations}

We first describe the analyses of the IAT-effects at pretest for the whole sample, then the analyses of the change in implicit associations as a function of treatment.

Implicit Associations at Pretest. As an initial analysis, we subjected the four IAT scores at pretest to a 2 (IAT-type, CR/RC) $\times 2$ (Affective-Dimension, Valence/Arousal) x 2 (IAT-Order, CR-RC/RC-CR) x 2 (Affect-order) Mixed ANOVA (the first two are within-subject factors, the others between-subject factors). There were large main effects for IAT-type, $F(1,88)=169, p<0.001$ and affective dimension, $F(1,88)=$ $24.9, p<0.001$, superseded by interactions between affective dimension and affective order, $F(1,88)=17.8, p<.001$, and between IAT-type, affective dimension and affective order, $F(1,88)=23.7, p<.001$. These effects are illustrated in Figure 1. It can be seen that the valence IAT produced larger effects than the arousal IAT, and that the CR-IAT resulted in larger effects than the RC-IAT. For the arousal-IAT, only the CR-version yielded a significant effect. These effects were enhanced by IAT order effects: when a participant started with the arousal-IAT, strong alcohol-arousal associations were found for the CR-IAT ( $81 \mathrm{~ms}$.), but not for the RC-IAT (15 ms.).

There was one other significant interaction: IAT $x$ type with IAT Order and with affective dimension, $F(1,88)=7.31, p=.008$. Implicit arousal associations in the CRIAT were much larger in the CR-RC condition (144 ms.) than in the RC-CR condition (24 ms.). Even though the size of the IAT effects appeared to be strongly dependent on order of assessment, the relative effects (individual differences) were stable, as indicated by the pattern of correlations: the CR and RC-IAT correlated 0.69 for Valence, and 0.64 for Arousal.

Change in Implicit Associations. The IAT-data of one participant were lost due to a technical problem. One influential outlier on the IAT-scores at post-test was removed, leaving 90 participants in these analyses (45 per condition). Type-l errors were reduced by directly testing the hypothesized interaction of condition and time (Tabachnick \& Fidell, 2001).

Implicit arousal associations. Given the analyses of the pretest data, we decided to consider only the CR-IAT arousal data (for which significant alcohol-arousal associations were found). The CR-Arousal-IAT scores at post-test were subjected to a 2 (Condition) $\times 2$ (Gender) $\times 2$ (IAT Order) $\times 2$ (affect order) ANCOVA, with pretest scores as covariate (more power than a repeated-measures ANOVA, Laird, 1983). There was no significant effect of Condition $(p>0.25)$. Results using the original IATalgorithm showed a different outcome: the Condition effect was significant, $F(1,73)$ $=4.82, p=0.031$. 


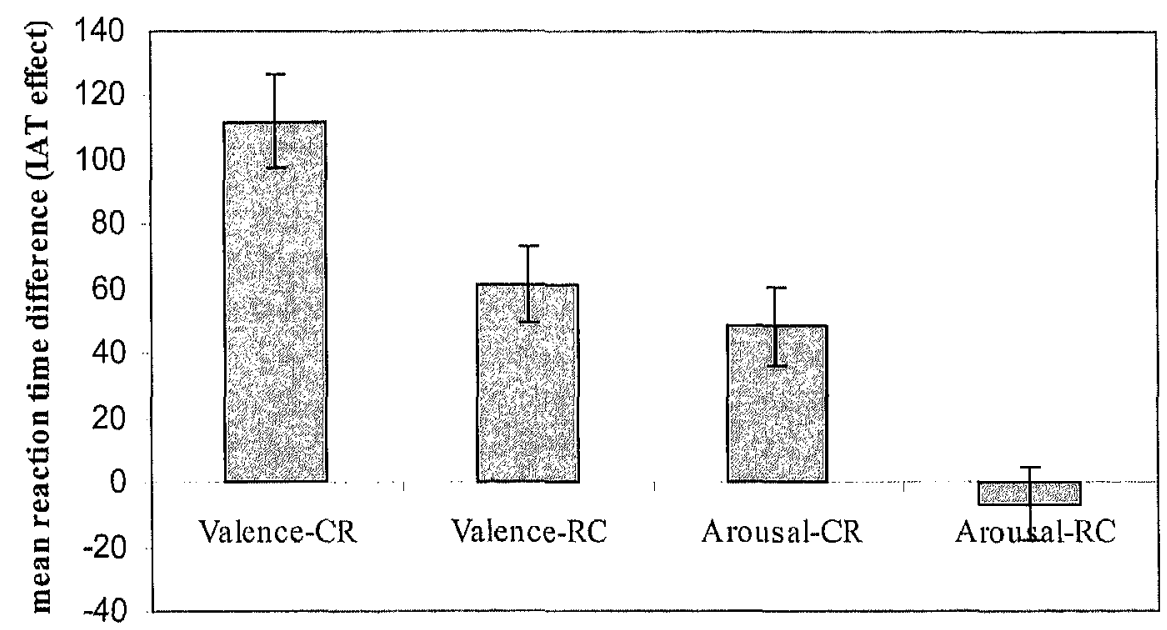

Figure 1: IAT-effects at pre-test for the two emotional dimensions valence and arousal, per IAT-version (CR or RC). IAT-effects are represented as difference in the reaction times between the Compatible (" $\mathrm{C}^{\prime \prime}$ ) phases and the Reverse Compatible ("R") phases. Compatible phases generate the fastest responses, here alcohol-negative (and soda-positive) for the valence-IAT and alcohol-arousal (and soda-sedation) for the arousal-IAT. In the CR-versions of the task, the Compatible phase comes first, followed by the Reverse compatible phase. All participants performed both a CR and a RC-IAT for both affective dimensions (see Table 2). This was done both at pre- and at post-test. The CR-IATs generally generate stronger IATeffects, and that for the arousal-IAT, only in the CR-IAT significant alcohol-arousal associations were found.

Follow-up analyses indicated that participants showed no difference at pretest $(p>$ $0.50)$. Participants in the EC condition showed a larger decrease in implicit alcoholarousal associations at post-test (EC-group changed from 45 to $17 \mathrm{~ms}$., Controls from 53 to 50 ms., Table 3).

The implicit attitudes were not expected to change as a function of treatment. This was analysed with the same ANCOVA, now on the implicit valence-IAT effects at post-test with pre-test scores as covariate. There was no significant effect of Condition ( $\left.p^{\prime} \mathrm{s}>0.19\right)$.

The second 2 (Time) $\times 2$ (Gender) $\times 2$ (Condition) Mixed MANOVA contained the explicit variables that were not expected to change differentially as a result of the EC: attitudes, negative expectancies, and negative reinforcement expectancies. The effect of interest (Time $x$ Condition) was not significant, $p>0.40$, nor any higher order-interaction involving these factors.

Because the pattern of results was the same for the implicit arousal associations (CR-IAT) and for the explicit arousal expectancies, we tested whether these changes were correlated, using residual gain scores (cf. Teachman \& Woody, 2003). This was not the case $(r=0.01, p>0.50)$. 
Table 3: Implicit and explicit cognitive variables before and after the intervention

\begin{tabular}{|c|c|c|c|c|c|c|c|c|}
\hline \multirow[b]{3}{*}{ Variable } & \multicolumn{4}{|c|}{ Expectancy Challenge } & \multicolumn{4}{|c|}{ Control Condition } \\
\hline & \multicolumn{4}{|c|}{$T_{0}$ (before intervention) $T_{1}$ (after intervention) } & \multicolumn{2}{|c|}{$\mathrm{T}_{0}$ (before intervention) } & \multicolumn{2}{|c|}{$T_{1}$ (after intervention) } \\
\hline & $M$ & $S D$ & $M$ & $S D$ & $M$ & $S D$ & $M$ & SD \\
\hline Valence IAT-CR (ms) & 142.52 & 150.26 & 81.34 & 86.43 & 76.75 & 116.6 & 48.17 & 81.21 \\
\hline Valence IAT-RC (ms) & 81.15 & 115.76 & 69.57 & 82.70 & 37.43 & 106.76 & 20.45 & 81.71 \\
\hline Arousal IAT-CR (ms) & 44.81 & 127.62 & 16.56 & 94.30 & 52.64 & 111.60 & 50.38 & 74.84 \\
\hline Arousal IAT-RC (ms) & -15.98 & 111.49 & 7.34 & 90.42 & 7.63 & 109.03 & -5.08 & 87.06 \\
\hline Valence IAT-CR (D) & .61 & .42 & .53 & .44 & .43 & .41 & .35 & .45 \\
\hline Valence IAT-RC $\{D\rangle$ & .30 & .37 & .31 & .47 & .16 & .38 & .12 & .41 \\
\hline Arousal IAT-CR (D) & .22 & .49 & .21 & .47 & .28 & .39 & .33 & .41 \\
\hline Arousal IAT-RC (D) & -.10 & .45 & -.01 & .46 & -.08 & .36 & -.03 & .40 \\
\hline VAS-Arousal & 67.6 & 17.4 & 63.1 & 11.1 & 69.3 & 16.1 & 71.7 & 14.0 \\
\hline VAS-Sedation & 47.2 & 17.8 & 53.4 & 13.0 & 47.9 & 13.3 & 48.8 & 11.0 \\
\hline VAS-Attitudes & 66.6 & 11.0 & 68.6 & 10.8 & 68.0 & 11.5 & 68.8 & 12.6 \\
\hline Pos. Reinf. Exp. & 3.34 & .59 & 3.13 & .61 & 3.33 & .60 & 3.25 & .60 \\
\hline Neg, Reinf. Exp. & 2.40 & .68 & 2.09 & .64 & 2.43 & .58 & 2.25 & .68 \\
\hline Neg. Exp & 1.55 & .34 & 1.57 & .36 & 1.61 & .42 & 1.60 & .40 \\
\hline
\end{tabular}

\subsubsection{Alcohol use}

One month follow-up. Weekly alcohol consumption and the number of binges per week showed significant departures from normality. Inspection of the data indicated that there were influential outliers. Inspection of the drinking diaries confirmed their validity (e.g. as a context for a heavy drinking episode, 'party' was indicated). The analyses were continued with the raw data and checked using a bootstrap regression analysis that makes no assumption for the distribution (see below).

Weekly alcohol consumption was analysed in a 4 (Time) $\times 2$ (Condition) $\times 2$ (Gender) mixed ANCOVA, with weekly drinking during post-test as within-subjects variables and pretest values of weekly consumption as covariate. The Time $x$ Condition - Gender interaction was significant, $F_{\text {Greenhouse-Geisser }(G G)}(2.9,249)=3.91, p=.01$, and the same was found for binges per week, $F_{G G}(2.9,248)=4.20, p=.007$. Analyses were split for gender.

For men, there was a significant Time $\mathrm{x}$ Condition effect for both variables: weekly consumption: $F_{G G}(2.8,118)=3.77, p=.014$; binges $F_{G G}(2.9,121)=3.99, p=$ .01 in the absence of time main-effects $(p$ 's $>20$ ). Figure 2 indicates that alcohol use in the EC first increased and then decreased compared with the controls (same pattern for binges). Between-subject ANCOVAs for each of the four time-points, indicated that the difference in weekly consumption was marginally significant in week $3, F(1,42)=4.08, p=.05$, but not during other time-points ( $p$ 's $>.10)$. Binges showed trends in week $2(p=.08)$, and in week $3(p=.09)$. More powerful bootstrap analyses (described below) confirmed the significance effect of the difference in alcohol use and number of binges in week $3(p<.01)$ in the absence of significant effects in other weeks. Note that analyses using the average weekly consumption or 
binges at posttest (controlling for pretest) did not indicate a significant time $\mathrm{x}$ condition effect.

For women, there was no significant Time $\mathrm{x}$ Condition effect for both variables ( $p^{\prime} s>30$ ). For weekly alcohol consumption (but not for binges), there was a significant time effect, indicating that alcohol use decreased in both conditions, $F_{G G}(2.8$, 120) $=2.97, p=.034$.

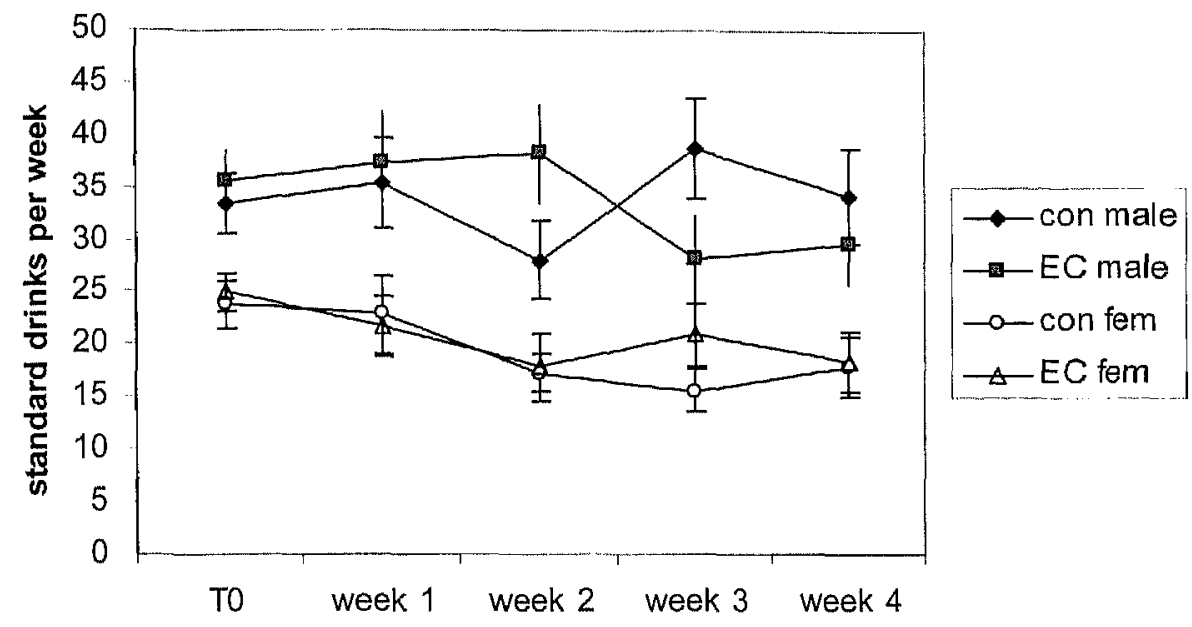

Figure 2: Weekly alcohol use in the four weeks after the expectancy challenge ("EC") or Control condition ("con") in male and female ("Fem") heavy drinkers, after controlling for baseline levels of weekly consumption and number of binges.

\subsubsection{Correlations}

Correlations between dependent variables are presented in Table 2. The general pattern was that the implicit measures correlated and that most explicit measures of alcohol-related cognitions correlated, with few correlations between these two clusters. One correlation is remarkable: implicit arousal associations correlated significantly with tension reduction expectancies. Implicit arousal associations correlated with alcohol problems, as did explicit negative expectancies and tension reduction expectancies.

\subsubsection{Mediation}

A prerequisite for mediation analysis is that the intervention differentially changes the mediator and the outcome-variable (Baron \& Kenny, 1986). It was tested whether the EC-induced reduction in arousal expectancies mediated the significant reduction in alcohol use in men in week 3 , using a model specifically proposed for the present design (MacKinnon, 1994). In this model, condition is an independent variable, the change in arousal expectancies the mediator and alcohol use the de- 
pendent variable. To test for the possibility that the inclusion of the control group suppressed a mediation effect (Shrout \& Bolger, 2002), we included the interaction term into the regression model (Cohen \& Cohen, 1983). Because the mediation analysis was performed in men only $(n=46)$, its power was limited. In these circumstances a bootstrap method is advised (Shrout \& Bolger, 2002). This approach has the advantage that the typical non-normal distribution of alcohol use is not problematic. A recent simulation study comparing different methods found that the biascorrected bootstrap method has the best power to detect mediation effects and is the current 'method of choice' (Mackinnon, Lockwood \& Williams, 2004). Mediation was tested for weekly alcohol use and weekly binges during follow-up (Figure 3, Table 4).

\begin{tabular}{|c|c|c|c|c|c|c|c|c|c|c|c|}
\hline \multirow[t]{2}{*}{ Mediator } & \multirow[t]{2}{*}{ Y } & \multirow[t]{2}{*}{ Time } & \multirow[t]{2}{*}{$a$} & \multirow[t]{2}{*}{ SE(a) } & \multirow[t]{2}{*}{$p$} & \multirow[t]{2}{*}{$b$} & \multirow[t]{2}{*}{$S E(b)$} & \multirow[t]{2}{*}{$p$} & \multirow{2}{*}{$\begin{array}{c}\text { Low } 95 \\
\mathrm{CL}\end{array}$} & Up 95 & \multirow[t]{2}{*}{$p$} \\
\hline & & & & & & & & & & $\mathrm{CL}$ & \\
\hline Change in Arousal & Binge & t3 & -0.399 & 0.132 & .003 & .787 & .314 & .012 & & & \\
\hline \multirow[t]{3}{*}{ Expectancies } & & t4 & -0.399 & 0.132 & .003 & .577 & .332 & .059 & & & \\
\hline & Alc Use & t3 & -0.399 & 0.132 & .003 & 11.26 & 4.84 & .020 & & & \\
\hline & & t4 & -0.399 & 0.132 & .003 & 8.67 & 4.20 & .039 & & & \\
\hline Change in Arousal $\exp x$ & Binge & t3 & -.059 & .029 & .042 & -1.146 & .523 & .028 & -.75 & -.069 & .030 \\
\hline \multirow[t]{3}{*}{ Condition (interaction) } & & t4 & -.059 & .029 & .042 & -1.04 & .551 & .059 & -.647 & .020 & .14 \\
\hline & Alc Use & $\mathrm{t} 3$ & -.059 & .029 & .042 & -12.30 & 7.86 & .12 & -9.90 & -.95 & .035 \\
\hline & & t4 & -.059 & .029 & .042 & -18.60 & 6.81 & .006 & -9.93 & .35 & .16 \\
\hline
\end{tabular}

The indirect effect of Condition on alcohol use in week 3 was significant $(p=.030)$, in the presence of a direct pathway from Condition to Use in week $3(p=.014)$. The change in arousal expectancies was a significant mediator of alcohol use in week 3 , indicating partial mediation. The indirect effect of Condition on binges in week 3 was significant $(p=.030)$, as was the direct effect $(p=.002)$. Both the change in arousal expectancies and the interaction of this change with condition were significant mediators (Table 4, Figure 3B).

\subsubsection{Non-Specific Effects}

Participants in the EC scored higher than participants in the control-group on two questions of the SRQ: usefulness of the information $(p<.001)$ and potential influence on drinking $(p<.001)$. The EC group felt slightly more pressure to change $(p=$ .09; both groups scored very low, <2). 


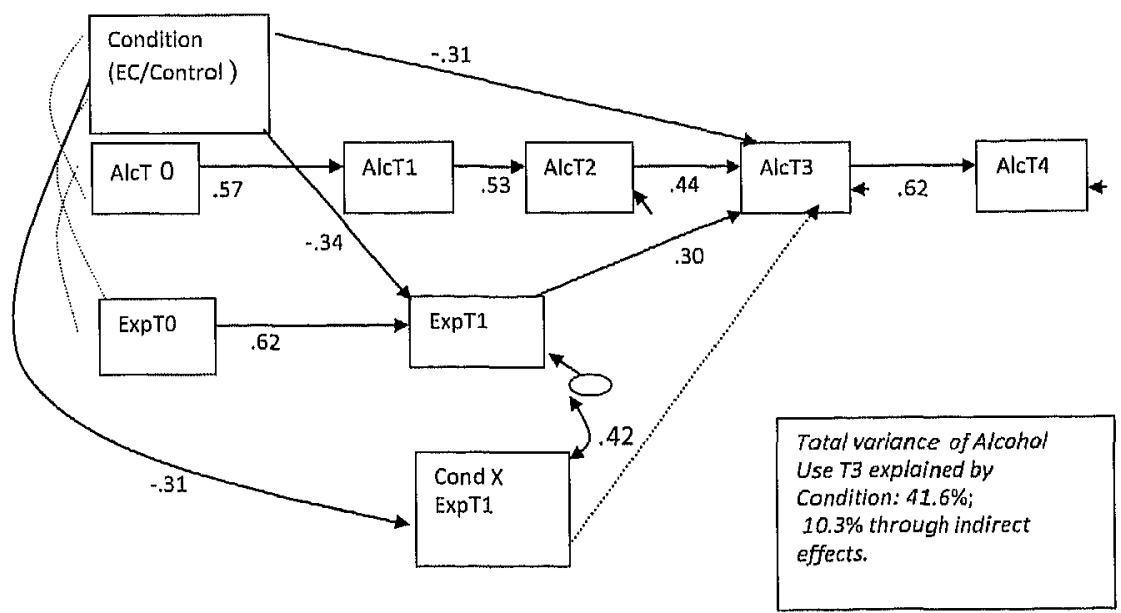

Figure 3a: Mediation Model Alcohol Use Week 3 Posttest (Standardized Bootstrap Estimates)

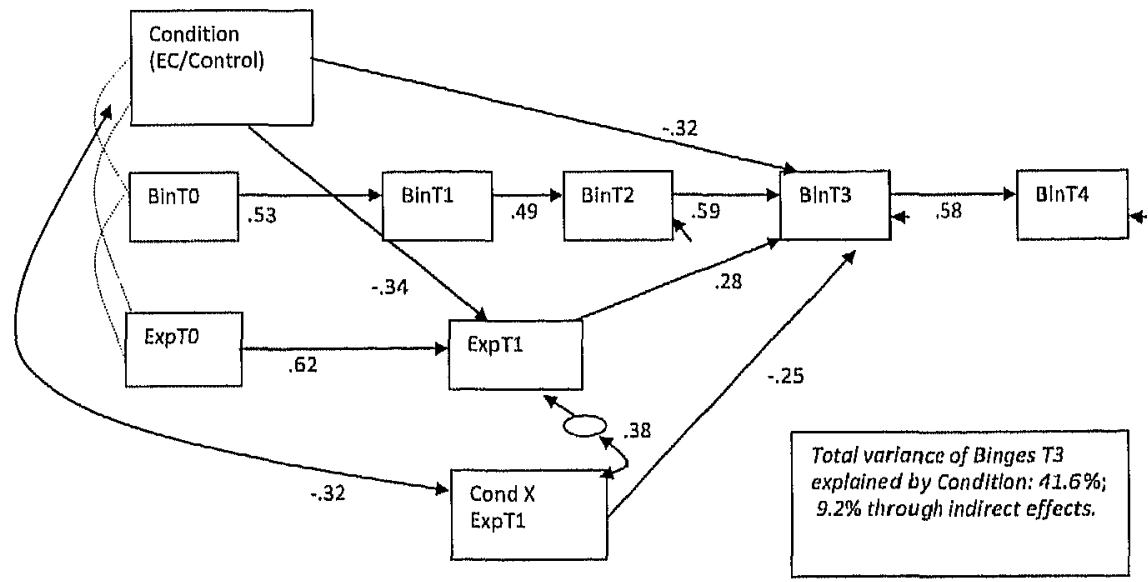

Figure 3b: Mediation Model Binges Week 3 Posttest (Standardized Bootstrap Estimates)

Figure 3: Mediation analysis of weekly alcohol use in men after the intervention. The model tested to what extent the experimentally induced change in explicit arousal expectancies mediated changes in prospective drinking in men, using bias corrected maximum likelihood bootstrap estimation, which has optimal power for the present sample size. This was done for weekly alcohol use (a) and for weekly number of binges (b). Drawn lines depict significant paths, dashed lines non-significant paths. Standardized regression weights are presented for significant paths and for dashed paths that showed a statistical trend $(0.05<P<0.10)$. Alc $=$ alcohol use in standard drinks; $\operatorname{Exp}=$ explicit arousal expectancles; $\mathrm{TO}=$ pretest, $\mathrm{T} 1=$ week 1 after the Intervention, $\mathrm{T}_{2}=$ week 2 after the intervention, etc. 


\subsection{Discussion}

This study investigated the effects of an extended single-session EC on implicit and explicit alcohol-related cognitions and prospective alcohol use in hazardous drinkers. The results can be summarized as follows: first, the EC successfully changed targeted positive arousal expectancies in men and women alike, while non-targeted explicit cognitions (negative expectancies, attitudes) did not differentially change. Secondly, for the first time implicit measures were included in an EC; two adapted versions of the implicit association test (IAT) were used in this research. Results at pre-test were in line with earlier findings with these measures (Wiers et al., 2002; De Houwer et al., 2004): heavy drinkers showed strong negative implicit alcohol associations and alcohol-arousal associations (here only on the CR-IAT). The EC differentially reduced the implicit arousal associations as assessed with this version of the IAT, but only when the original scoring algorithm was used. Thirdly, the EC differentially changed alcohol use in men, but the effect was delayed (week 3 postintervention) and short-lived (no longer significant in week 4). Fourthly, for the first time the hypothesized mechanism of an EC was formally tested with mediation analyses. The decrease of alcohol use in men three weeks after the EC was mediated partially by the change in explicit arousal expectancies. These findings raise a number of theoretical and practical issues.

\subsubsection{Assessing and Changing Implicit Alcohol Associations}

Although the findings using the alcohol-lAT have been consistent and reliable (cf. Wiers et al., 2002; De Houwer, 2003), the external validity of IAT has been criticized (e.g. De Houwer, 2002; Fazio \& Olsen, 2003). One concern is the bipolar nature of the IAT. Note that in the IAT, both the target categories (alcohol-soda) and the attribute categories (positive-negative or arousal-sedation) are bipolar. First, with respect to the target categories, the alcohol-associations measured in IATs used here are relative to soda-associations (hence, negative alcohol associations could theoretically be due to positive soda associations). Secondly, the attribute dimensions are also bipolar. This may be problematic in the context of alcohol: for explicit measures there is evidence that people hold both positive and negative expectancies (e.g. Leigh, 1989) and the same may be true for implicit associations (Jajodia \& Earleywine, 2003; Kramer \& Goldman, 2003). In a recent study, associations for the four attribute categories used here were compared in a balanced series of unipolar IATs. It was found that negative associations were strongest $(d>1)$, followed by positive and arousal associations ( $d$ approximately .8 ) and sedation associations ( $d$ approximately .5), and this was the case both for the soda contrast and for an irrelevant animal-contrast (Houben \& Wiers, 2004). These findings are consistent with the findings using bipolar IATs: stronger negative than positive alcohol-associations, and stronger arousal than sedation alcohol associations (Wiers et al., 2002; De Houwer et al., 2004; this study). However, the findings of strong negative alcohol associations are inconsistent with assessments with explicit measures (Goldman et al., 1999; Wiers et al., 2002; this study). The strong negative alcohol-associations found with 
the IAT could be related to an aspect of the IAT-assessment procedure (e.g. Rothermund \& Wentura, 2004), or could be meaningful and represent negative experiences with alcohol (e.g. Jones \& McMahon, 1998; Rudman, 2004). In line with the latter view, negative associations have also been found with memory associations (Gadon et al., 2004). The implicit (positive) arousal associations for alcohol found with the IAT are consistent with other research using the IAT (Wiers et al., 2002; De Houwer et al., 2004), and with other implicit and explicit methods (Goldman et al., 1999; Dunn et al., 2000; Kramer \& Goldman, 2003). The fact that they were found only with one version of the IAT and were sensitive to order effects may have attenuated effects of the EC on implicit alcohol-arousal associations. In future treatment studies it might be wise to use only this version or a unipolar IAT. The finding that the expected decrease in implicit alcohol-arousal associations was found only for the original but not for the new scoring-algorithm (Greenwald et al., 2003) may either indicate that the finding here is not reliable or that the new algorithm is less appropriate for intervention-studies (the individual normalization procedure could take away variance crucial for detecting change). Clearly these issues require further research. The present study adds to the growing evidence that implicit and explicit measures assess different constructs (Stacy, 1997; Wiers et al., 2002; Rudman, 2004), and that interventions may differentially affect implicit and explicit cognitions (cf. Teachman \& Woody, 2003). Therefore, it is important to assess effects of interventions on both types of measures and to develop new ways to change implicit associations (Wiers et al., 2004).

\subsubsection{A causal role for explicit expectancies?}

The EC successfully reduced the explicit expectancies related to arousal and positive reinforcement in men and women. The effect size was equal ( $d=0.67)$ to the original study using multiple sessions (Darkes \& Goldman, 1993), supporting the validity of the adapted EC and control condition in changing expectancies. Further, it was confirmed that the adapted protocol changed expectancies in women too (Wiers \& Kummeling, 2004). The expected change in heavy drinking was found in men, but only in week 3 after the EC (not in other weeks, nor in the overall follow-up month). A delayed effect could be related to the homework assignment (albeit post hoc). Alternatively, the short-lived significant reduction in alcohol use could be a chance finding. In women, no differential effects of the $\mathrm{EC}$ were found on drinking variables despite similar effects on explicit and implicit arousal expectancies, which could be interpreted as a disconfirmation of the hypothesized causal role of expectancles (Jones et al., 2001a,b). An alternative could be that the control condition was somehow equally effective in reducing drinking in women (not mediated by expectancies). The change in explicit arousal expectancies in men significantly mediated the short-lived delay in alcohol use, which is the first empirical confirmation of the hypothesized mediational role of expectancies (cf. Goldman, 1999; Goldman et al., 1999; Jones et al., 2001a, b; Wiers 2002a), although the validity of this finding depends on the interpretation of the significant difference in week 3 as meaningful or a chance. 


\subsubsection{Limitations}

An experimental design was chosen that inciuded an active control group instead of the more usual assessment-only control group. The advantage of our choice was that an effect of the EC could be related more specifically to the intervention and that non-specific context effects could be excluded as alternative explanations for the effects found. The drawbacks are that a comparison with studies using the more usual passive control group is more difficult and that a potential effect of the EC on drinking in women may have been obscured by an effect on drinking in the controlgroup (not mediated by expectancies). Future studies could include both control conditions. Finally, assessment-strategies for implicit (alcohol) associations are developing rapidly and it is possible that implicit associations assessed with a different method would show stronger effects in an EC.

\subsubsection{Practical Implications}

There is now quite some evidence for short-term reductions of alcohol consumption in men following an EC (Darkes \& Goldman, 1993; 1998; Dunn et al., 2000; this stu$d y$ ), but little evidence for its effect on drinking in women (although our adapted version consistently changed expectancies in women). The practical utility of the EC may be that it is an attractive group-intervention for young problem drinkers who are typically unaware of their alcohol problem. In this way, it may provide a lowthreshold initial contact that could be followed-up with a motivational interview (cf. Del Boca et al., 2002; Wiers \& Kummeling, 2004), for which longer-term effects on alcohol use have been established (e.g. Marlatt et al., 1998; Baer et al., 2001). In addition, prevention efforts aimed at the environment rather than at the individual hold promise in binge-drinking students (e.g. DeJong \& Langford, 2002).

\subsubsection{Conclusion}

An adapted EC reduced explicit positive-arousal expectancies in men and women in the absence of such changes in the control condition. The effects of the EC on implicit alcohol associations were less clear-cut and need further investigation. There is accumulating evidence that the EC results in modest, short-term reductions in alcohol consumption in heavy drinking young men, which was found here to be partially mediated by the EC-induced change in explicit positive arousal expectancies. Key questions for further research are how implicit alcohol associations can be changed succesfully, and which combination of preventive interventions results best in longterm changes in young problem drinkers. 


\section{References}

Baer, J. S., Kivlahan, D. R., Blume, A. W., Mc Knight, P. \& Marlatt, G. A. (2001). Brief intervention for heavy-drinking college students: 4-year follow-up and natural history. American-Journal of Public Heaith, 91, 1310-1316.

Baron, R. M. \& Kenny, D. A. (1986). The moderator-mediator variable distinction in social psychological research: conceptual, strategic, and statistical considerations. Journal of Personality and Social $p_{s y-}$ chology, 51, 1173-1182.

Bechara, A., Damasio, A. R. \& Damasio, H. (2003). The role of the amygdale in decision-making. Annals of the NY academy of science, 958, 356-369.

Bijl, R. V., Ravelli, A. \& van Zessen, G. (1998). Prevalence of psychiatric disorder in the general population: Results of the Netherlands Mental Health Survey and Incidence Study (NEMESIS). Social Psychlatry and Psychiatric Epidemiology, 33, 587-595.

Blair, I.V. (2002). The malleability of automatic stereotypes and prejudice. Personality and Social Psychology Review, 6, 242-261.

Bosson, J. K., Swann, W. B. \& Pennebaker, J. (2000). Stalking the perfect measures of implicit self-esteem: the blind man and the elephant revisited? Journal of Personality and Sacial Psychology, 79, 631-643.

Cohen, J. \& Cohen, P. (1983). Applied multiple regression/correlation analysis for the behavioral sciences. (Znd Ed.). Hillsdale NJ: Erlbaum.

Cunningham, W. A. (2003). Neural components of implicit and explicit social evaluation. Dissertation Abstracts International: Section B: The Sciences and Engineering, 64, 1548.

Darkes, J. \& Goidman, M. S. (1993). Expectancy challenge and drinking reduction: experimental evidence for a mediational process. Journal of Consulting and Clinical Psychology, 61, 344-353.

Darkes, J. \& Goldman, M. S. (1998). Expectancy challenge and drinking reduction: process and structure in the alcohol expectancy network. Experimental and Clinical Psychopharmacology, 6, 64-76.

De Houwer, J. (2002). The Implicit Association Test as a tool for studying dysfunctlonal associations in psychopathology: strengths and limitations. Journal of Behavior Therapy and Experimental Psychiatry, 33, 115-133.

De Houwer, J., Crombez, G., Koster, E. H. W. \& De Beul, N. (2004). Implicit alcohol-related cognitions in clinical samples of heavy drinkers. Journal of Behavior Therapy and Experimental Psychiatry, 35(4), 275-286.

De Jong, W. \& Langford, L. M. (2002). A typology for campus-based alcohol prevention: moving toward environmental management strategies. Journal of Studies on Alcohol, Supplement 14, 140-147.

Del Boca, F. K. \& Darkes, J. (2001). Is the glass half full or half empty? An evaluation of the status of expectancies as causal agents. Addiction, 96, 1670-1672.

Del Boca, F. K., Darkes, J., Goldman, M. S. \& Smith, G. T. (2002). Advancing the expectancy concept via the interplay between theory and research. Alcoholism: Clinical and Experimental Research, 26, 926 935.

Dimeff, L.A., Baer, J.S., Kivlahan, D.R., Marlatt, G.A. (1999). Brlef Alcohol Screening and Intervention for College Students (BASICS): A harm reduction approach. NY: Guilford.

Dunn, M. E., Lau, H. C. \& Cruz, I. Y. (2000). Changes in activation of alcohol expectancies in memory in relation to changes in alcohol use after particlpation in an expectancy challenge program. Experimental and Clinical Psychopharmacology, 8, 566-575.

Fazio, R. H. \& Olson, M. A. (2003). Implicit measures in social cognition research: their meaning and use. Annual Review of Psychology, 54, 297-327.

Gadon, L., Bruce, G., McConnochie, F. \& Jones, B. T. (2004). Negative alcohol consumption outcome associations in young and mature adult social drinkers: a route to drinking restraint? Addictive Behaviors, 29, 1373-1387.

Goldman, M. S., Del Boca, F. K. \& Darkes, J. (1999). Alcohol expectancy theory: the application of cognitive neuroscience, In H. T. Blane, \& K. E. Leonard (Eds.), Psychological theories of drinking and alcohoIism (2nd ed.). pp. 203-246. NY: Guilford. 
Greenwald A. G. \& Nosek, B. A. (2001). Health of the IAT at age 3. Zeitschrift fur Experimentelle Psychologie (Experimental Psychology), 48, 85-93.

Greenwald, A. G. \& Banaji, M. R. (1995). Implicit social cognition: attitudes, self-esteem, and stereotypes. Psychological Bulletin, 102, 4-27.

Greenwald, A. G., McGhee, D. E. \& Schwartz, J. L. K. (1998). Measuring individual differences in implicit cognition. The implicit association test. Journal of Personality and Social Psychology, 74, 1464-1480.

Greenwald, A. G., Nosek, B. A. \& Banaji, M. R. (2003\}. Understanding and using the implicit association test: I An improved scoring algorithm. Journal of Personality and Social Psychology, 85, 197-216.

Hingson, R., Heeren, T. Zakocs, R., Kopstein, A. \& Wechsier, H. (2002). Magnitude of Alcohol-Related Mortality and Morbidity among U.S. College Students Ages 18-24. Journal of Studies on Alcohol, 63, 136-144.

Houben, K. \& Wiers, R. W. (2004). Implicit alcohol associations: influence of target category labels and contrast categories in a unipolar IAT. Alcoholism, Clinical and Experimental Research, 28 (Supplement), 102A (Abstract).

Huberty, C. J. \& Morris, J. D. (1989). Multivariate analysis versus multiple univariate analysis. Psychologicol Bulletin, 105, 302-308.

lajodia, A. \& Earleywine, M. (2003). Measuring alcohol expectancies with the Implicit Association Test. Psychology of Addictive Behaviors, 17, 126-133.

Jones, B. T., \& McMahon, J. (1998). Alcohol motivations as outcome expectancies. In: W. R. Miller \& N. Heather (Eds). Treating addictive behaviors (2nd ed.). Applied clinical psychology. pp. 75-91. New York, NY, US: Plenum Press.

Jones, B. T., Corbin, W. \& Fromme, K. (2001a). A review of expectancy theory and alcohol consumption. Addiction, 91, 57-72.

Jones, B. T., Corbin, W. \& Fromme, K. (2001b). Half full or half empty, the glass still does not satisfactorily quench the thirst for knowledge on alcohol expectancies as a mechanism of change. Addiction, 96, 1672-1674.

Kramer, D. A. \& Goldman, M. S. (2003). Using a modified Stroop task to implicitly discern the cognitive organization of alcohol expectancies. Journal of Abnormal Psychology, 112, 171-175.

Laird, N (1983). Further comparative analyses of pretest-posttest research designs. American Statistician, $37,329-330$.

Larimer, M. E. \& Cronce, J. M. (2002). Identification, prevention, and treatment: a review of individualfocused strategies to reduce problematic aicohol consumption by college students. Journal of Studies on Alcohol, Supplement 14, 148-163.

Leigh, B. C. (1989). In search of the seven dwarves: issues of measurement and meaning in alcohol expectancy research. Psychological Bulletin, 105, 361-373.

Maalsté, N. (2000). Ad Fundum! Een blik in de gevarieerde drinkcultuur van het Nederlandse studentenleven [Ad Fundurn! A view on the diverse drinking culture of Dutch Students]. Utrecht, The Netherlands; CVO.

Mackinnon, D. P. (1994). Analysis of mediating variables in prevention and intervention research. In: A. Cazares \& L. A. Beatty (Eds.). Scientific methods for prevention intervention research. pp. 127-153. NIDA Research Monograph No 139. NIH Publication 94-3631. Washington DC: Government printing office.

Mackinnon, D. P., Lockwood, C. M. \& Williams, J. (2004). Confidence limits for the indirect effects. Distribution of the Product and Resampling Methods. Mutivariate Behavioral Research, 39(1), 99-128.

Marlatt, G. A. \& Rohsenow, D. J. (1980). Cognitive processes in alcohol use: expectancy and the balanced placebo design. In N. K. Mello (Ed.), Advances in substance abuse: behavioral and biological research. pp. 159-199. Greenwich: JAI Press.

Marlatt, G. A., Baer, J. S., Kivlahan, D. R., Dimeff, L. A., Larimer, M. E., Quigley, L. A., et al. (1998). Screening and brief intervention for high-risk college student drinkers: results from a 2-year follow-up assessment. Journal of Consulting and Clinical Psychology, 66, 604-615. 
Musher-Eizenman, D. R. \& Kulick, A. D. (2003). An alcohol expectancy-challenge prevention program for at-risk college women. Psychology of Addictive Behaviors, 17, 163-166.

Palfai, T. P. \& Ostafin, B. D. (2003). Alcohol-related motivational tendencies in hazardous drinkers: Assessing implicit response tendencies using the modified IAT. Behaviour Research and Therapy, 41, 1.1491162.

Phelps, E. A., O'Connor, K. J., Cunningham, W. A., Funayama, E., Gatenby, J., Gore, J. C., et al. (2000). Performance on indirect measures of race evaluation predicts amygdala activation. Journal of Cognitive Neuroscience, 12, 729-738.

Robinson, T. E. \& Berridge, K.C. (2001). Mechanisms of action of addictive stimuli. Incentive-Sensitization and addiction. Addiction, 96, 103-114.

Rothermund, K. \& Wentura, D. (2004). Underlying processes in the Implicit Association Test (IAT): Dissociating salience from associations. Journal of Experimental Psychology: General, 133, 139-165.

Rudman, L. A. (2004). Sources of implicit attitudes. Current Directions in Psychological Science, 13, 79-82.

Saunders, J. B., Aasland, O. G., Babor, T. F., De la Fuente, J. R., \& Grant, M. (1993). Development of the Alcohol Use Disorders Identification Test (AUDIT): WHO collaborative project on early detection of persons with harmful alcohol consumption-II. Addiction, 88, 791-804.

Sherman, S. J., Presson, C. C., Chassin, L., Rose, J. S. \& Koch, K. (2003). Implicit and explicit attitudes toward cigarette smoking: The effects of context and motivation. Journal of Social and Clinical Psychology, 22, 13-39.

Shrout, P. E. \& Bolger, N. (2002). Mediation in experimental and nonexperimental studies: new procedures and recommendations, Psychological Methods, 7, 422-445.

Sobell, L. C. \& Sobell, M. B. (1990). Self-report issues in alcohol-abuse: state of the art and future directions. Behavioral Assessment, 12, 77-90.

Stacy, A. W. (1997). Memory activation and expectancy as prospective predictors of alcohol and marihuana use. Journal of Abnormal Psychology, 106, 61-73.

Tabachnick, B. G. \& Fidell, L. S. (2001). Using multivariate statistics (4th ed.). Needham Heights, MA: Allyn \& Bacon.

Teachman, B. A. \& Woody, S. R. (2003). Automatic processing in splder phobia: implicit fear associations over the course of treatment. Journal of Abnormal Psychology, 112, 100-109.

Wall, A., Mckee, S. A. Hinson, R. \& Goldstein, A. (2001). Examining alcohol outcome expectancies in laboratory and naturalistic bar settings: a within-subject experimental analysis. Psychology of Addictive Behaviors, 15, 219-226.

White, H. R. \& Labouvie, E. W. (1989). Towards the assessment of adolescent problem drinking. Journol of Studies on Alcohol, 50, 30-37.

White, H. R. \& Labouvie, E. W. (2000, June). Longitudinal trends in problem drinking as measured by the Rutgers Alcohol Problem Index. Alcoholism, Clinical and Experimental Research, 24 (Supplement), 76A (Abstract).

White, N. M. (1996). Addictive drugs as reinforcers: Multiple partial actions on memory systems, Addict/on, 91, 921-949.

Wiers R. W. (2002a). Half full or half empty, what are we drinking? Some comments on the discusslon of the causal role of alcohol expectancies as a mechanism of change. Addiction, 97, 599-600.

Wiers, R. W. (2002b). First associations with alcohol and the development of a short questionnaire to assess alcohol expectancies in two dimensions: arousal-sedation and positive-negative. Alcoholism, Clinical and Experimental Research, 26 (Supplement), 32A (Abstract).

Wiers, R. W., De Jong, P. J., Havermans, R., \& Jelicic, M. (2004). How to change impliclt drug-related cognitions in prevention: A transdisciplinary integration of findings from experimental psychopathology, social cognition, memory and learning psychology. Substance Use and Misuse, 39 (10-12), 1625-1684.

Wiers, R. W., Hoogeveen, K. J., Sergeant, J. A. \& Gunning, W. B. (1997). High and low dose expectancles and the differential associations with drinking in male and female adolescents and young adults. $A d-$ diction, $92,871-888$. 
Wiers R. W. \& Kummeling R. C. (2004). An Experimental Test of An Alcohol Expectancy Challenge in Mixed Gender Groups of Young Heavy Drinkers. Addictive Behaviors, 29, 215-220.

Wiers, R.W., \& Stacy, A.W. (2006). Handbook on Implicit Cognition and Addiction. Thousand Oaks, CA: Sage.

Wiers R. W., Van Woerden, N., Smulders, F. T. Y. \& De Jong, P. J. (2002). Implicit and Explicit AlcoholRelated Cognitions in Heavy and Light Drinkers. Journal of Abnormal Psychology, 111, 648-658.

Wiers, R. W., Wood, M. D., Darkes, J., Corbin, W. R., Jones, B. T. \& Sher, K. J. (2003). Changing expectancies: cognitive mechanisms and context effects. Alcoholism Clinical and Experimental Research, 27, 186-197.

Zack, M., Toneatto, T., \& MacLeod, C. M. (1999). Implicit activation of alcohol concepts by negative affective cues distinguishes between problem drinkers with high and low psychiatric distress. Journal of Abnormal Psychology, 108, 518-531. 


\section{CHAPTER 4}

\section{From the laboratory to real-life: A pilot study of an expectancy challenge with "heavy drinking" young people on holiday}




\begin{abstract}
The Alcohol Expectancy Challenge (EC, Darkes \& Goldman, 1993) is a promising program for changing alcohol expectancies and reducing alcohol consumption in "heavy drinking" young men in a bar-lab setting. In this study the EC was adapted for use in mixed-gender groups in a holiday setting and its feasibility tested in camping resorts in the Netherlands where a lot of binge-drinking takes place (summer 2002). Male and female participants ( $N=170$, mean age 18.8 years) were randomly assigned to an EC or to an assessment-only control group. One day before the intervention, alcohol expectancies were measured by a Visual Analogue Scale of arousalsedation expectancies (VAS-expectancies questionnaire). At the same time, alcohol use in everyday life and on holiday was assessed by a General Drinking Questionnaire and 24 hour Drinking Diary, respectively. Twenty-four hours after the intervention the VAS-expectancies questionnaire was administered again and alcohol use over the previous 24 hours was reported in the Drinking Diary. Six weeks after the intervention participants were telephoned and administered oral versions of the VAS-expectancies questionnaire and General Drinking Questionnaire. Data were analysed using mixed ANOVAs. Although the study was hampered by recruitment difficulties, the EC proved feasible in this setting, was well-received by youngsters and effects on their alcohol expectancies may have been present. No effect was found on alcohol use. In conclusion, implementation must be improved and more studies are needed to come to more definite conclusions about the value of the EC in a real-life targeted intervention.
\end{abstract}




\subsection{Introduction}

Many young people in the Netherlands binge alcohol while going out during the weekend. When on vacation without their parents, binge drinking occurs nearly every day (Lemmers, Willems, Thijssen, \& Osterman, 1998; Pos, Knibbe, \& Lemmers, 2001). This consumption pattern generally lasts their entire stay, usually a 1 to 2 week period. These youngsters face many health risks during such "heavy-drinking" episodes. Research by Van de Hoef, Lemmers and Knibbe (2001) showed that 45.2 $\%$ of young men experienced black-outs during their holiday, $32.3 \%$ had trembling hands and $\mathbf{1 2 . 2} \%$ had been involved in some sort of accident during their stay. Pos and colleagues (2001) interviewed key figures (e.g. campsite managers, bar owners, social workers) about the health problems young people experience when drinking excessively on holiday. Most key figures mentioned dealing with youngsters on holiday who experienced the consequences listed above. In addition, several key figures witnessed cases of alcohol-poisoning. Holiday with friends and without parents is seen as a 'time-out' situation in which normal behavioural rules do not apply' (Pos et al., 2001). It is similar to going out in the weekend, but even 'better' because school and parents are more than a few days away. With no parents to supervise them, no social responsibilities and few activities to be done, drinking is the main pastime.

The Dutch 'National Institute for Disease Prevention and Health Promotion' (NIGZ) has launched a 'summer campaign' in these seaside communities every year since 1998. Approximately 20.000 young people are reached by the campaign each year. Teams of trained students ("peers") approach youngsters (15-25 years) to talk about their alcohol consumption and inform them about alcohol. The theoretical underpinnings of this interpersonal intervention include the "Theory of Planned Behavior" (Ajzen, 1991). The aims of the summer campaign are to increase knowledge of the risks of excessive alcohol use and of guidelines for safe alcohol consumption. Other aims are to incite young people to think more about their own alcohol use and to talk about it more frequently. Although these goals are met, young people on holiday have not reduced their alcohol consumption (Lemmers et al., 1998). A literature survey showed that the Expectancy Challenge (EC) had promising results in reducing heavily drinking university students' positive alcohol expectancies and alcohol consumption (Larimer \& Cronce, 2002). It was noted that EC procedures might serve as prevention and intervention tool (Goldman, Del Boca, \& Darkes, 1999; Larimer \& Cronce, 2002). This led to the idea to implement and evaluate the EC as an intervention for young people on holiday. The study was initiated by researchers of Maastricht University in close collaboration with colleagues of the NIGZ, who were looking for new intervention methods to improve their summer campaign.

Many studies have shown correlations between alcohol expectancies and alcohol consumption (e.g. Goldman, 1999). "Heavy drinkers" hold relatively strong positive-arousal expectancies (Goldman et al., 1999). On the basis of this correlation, it was proposed that reducing positive arousal expectancies could lead to a reduction in alcohol consumption (Darkes \& Goldman, 1993; 1998). The EC is based on this 
hypothesis, it is designed to allow young people to find out for themselves that some of their positive alcohol expectancies are unrealistic and flawed.

Studies differ in the exact protocols used, but in the typical EC session, youngsters are brought together in a bar-like room, where they are given a glass of vodkatonic or placebo. A game is played during drinking. After everyone has finished drinking, they are asked to identify who had alcohol, based on observations of subjects' behaviour. Usually, many identification errors are made (participants indicate people who drank placebo as having drunk alcohol and vice versa). These errors are used by the session leader to separate expectancy and pharmacological effects of alcohol.

Several ECs have been evaluated to date. Darkes and Goldman (1993) were the first to manipulate alcohol expectancies in their 'social/sexual expectancy challenge'. Using a multiple-session protocol on male university students, they found significant reductions in expectancies and aicohol consumption. Since then, ECs have been adapted for use in mixed-gender and female-only groups (Corbin, McNair, \& Carter, 2001; Dunn, Lau, \& Cruz, 2000; L. Jones, Silvia, \& Richman, 1995; Maddock, Wood, Davidoff, Colby, \& Monti, 1999; Musher-Eizenman \& Kulick, 2003; Wiers \& Kummeling, 2004), information-only ECs have been conducted (Corbin et al., 2001; L. Jones et al., 1995) and single-session ECs have been performed (Corbin et al., 2001; L. Jones et al., 1995; Maddock et al., 1999; Wiers, Van de Luitgaarden, Van den Wildenberg, \& Smulders, 2005).

Many studies suggest the EC may not be effective in changing women's alcohol consumption (e.g., Corbin et al., 2001; Dunn et al., 2000; Musher-Eizenman \& Kulick, 2003; Wiers et al., 2003), however generalisation is hampered by differences in protocols used (e.g experiential vs. information-oniy, multi-session vs. singlesession, Wiers, 2002). Furthermore, Wiers and Kummeling (2004) did find reduced positive expectancies and a trend towards reduced alcohol use in women, in their mixed-gender multi-session program. More recently, Wiers et al. (2005) found again a significant reduction in positive expectancies in men and women alike, as a result of an EC (although in women no differential effect on drinking was found). In general, multiple session programs may result in larger effects on alcohol expectancies and consumption than single-session protocols (e.g., Darkes \& Goldman, 1993, 1998; Wiers, 2002). However, single-session ECs are better applicable in real-life intervention settings.

Performing an EC with young people on holiday is innovative in several respects. First, theories are rarely applied so directly to the intervention practice. To the best of our knowledge ECs have never been evaluated outside bar-labs of universities. In this study, the EC was performed on various locations near the campsites of "heavy drinking" youth. It is unique for a targeted intervention to take place in the actual situation in which binge-drinking occurs. Second, this is the first time the EC was done with such "heavy drinking" youth. Earlier evaluations mainly used students, who drank heavily on several occasions each week. However, most young people on holiday binge drink every day of their stay.

Because this is a first study of an EC outside the laboratory, the feasibility of an $E C$ on location was first evaluated. Second, we tested whether systematic differen- 
ces in expectancies arose between the experimental and control group as a result of the EC. Third, we explored if effects on alcohol use were present. Last, we explored gender differences.

\subsection{Method}

\subsubsection{Participants}

Young people (mean age 18.8 years; minimum age 18.0 years) spending a vacation at a seaside camping site were recruited by trained students ('peers'). The vast majority (88.1\%) were on holiday with friends, without their parents or partner. Their circle of friends included 8.2 persons on average (including themselves).

A total of 170 participants were recruited. However, many participants failed to show up for the intervention or could not be reached for the post-tests (see Table 1). After filling out a questionnaire and giving informed consent, participants were randomly assigned to one of two conditions (control or EC). Mean consumption at baseline was 23.1 Dutch standard-drinks (which contain \pm 10 grams of alcohol compared with 14 grams in the U.S.) per day during vacation, which can be classified as very "heavy drinking". Initially, the idea was to include equal numbers of males and females in each condition. However, young women were much more difficult to recruit than young males which led to an uneven distribution of $84 \%$ men $(n=142)$ and $16 \%$ women $(n=28)$ at pre-test.

Table 1: Number of participants per questionnaire, time, condition and gender

\begin{tabular}{llllllll}
\hline & & \multicolumn{5}{l}{ EC } & \multicolumn{3}{l}{ Control } \\
\cline { 3 - 7 } & Questionnaire & Men $(n)$ & Women $(n)$ & Men $(n)$ & Women $(n)$ & Total $(n)$ \\
\hline T1 & 24-hour Drinking Diary & 46 & 11 & 68 & 10 & 135 \\
& General Drinking Questionnaire & 33 & 9 & 66 & 10 & 118 \\
& VAS-Expectancies Questionnaire & 53 & 11 & 86 & 16 & 166 \\
\hline T2 & 24-hour Drinking Diary & 25 & 9 & 89 & 17 & 170 \\
\hline \multirow{2}{*}{ T3 } & VAS-Expectancies Questionnaire & 33 & 9 & 47 & 7 & 88 \\
& General Drinking Questionnaire & 29 & 9 & 63 & 10 & 115 \\
\hline
\end{tabular}

Note: T1: pretest measurements taken 1 day before the intervention. T2: measurements taken 24 hours after the intervention. T3: telephone interview six weeks after the intervention. Background questions addressed parents' drinking, education, peer group size, length of stay and romantic relationships.

Selective attrition? To examine if participants who did not complete all measurements differed from those who did, background characteristics of these two categories were compared on two points in time. First, attrition at the 1-day post-test was analysed using a logistic regression analysis. Attrition could not be predicted by condition, gender, age, alcohol expectancies or measures of alcohol use. Second, 
attrition at the six-week telephone interview was analysed. Condition predicted dropout at this point in time odds ratio $=3.836, p=.02$. Significantly more control participants did not complete the last measure.

\subsubsection{Measures}

Several questionnaires were used to measure if any changes in alcohol expectancies and alcohol use occurred.

Twenty-four hour Drinking Diary. After participants agreed to take part in the 'alcohol experiment' they were asked to fill out a drinking diary for the past 24 hours. In the drinking diary, they were asked if they had drank any alcohol in the previous 24 hours and when they had had alcohol, at which times they had drank, where the alcohol was consumed and how much standard glasses of alcohol they drank. Twenty-four hours after the EC participants (both controls and EC participants) reported their alcohol consumption of the last day.

General Drinking Questionnaire. To measure alcohol consumption in 'everyday life', participants answered questions about their alcohol consumption when not on vacation. Research shows that $80 \%$ of youngsters' alcohol consumption takes place when going out during the weekend (Knibbe, Oostveen, \& Van de Goor, 1991), therefore we asked how many alcoholic drinks they drank on typical week-days, frequency of going out in the weekend, number of glasses consumed while going out and number of glasses consumed prior to going out (at home or at a friend's house). In the results section, a combined measure of drinking while going out and drinking prior to going out is used.

Visual Analogue Scale of arousal-sedation expectancies (VAS-expectancies). The VAS-expectancies questionnaire was used to measure changes in expectancies that occurred after the intervention (Wiers et al., 2005). This questionnaire was chosen because it is relatively short, reduces memory-effects that can occur with repeated administrations (Schwartz, 1999), includes equal numbers of items describing desirable and undesirable effects (see Leigh \& Stacy, 1993) and was previously successful in detecting changes in expectancies as a result of an EC (Wiers et al., 2005). Table 2 shows test-retest reliabilities and internal consistencies of the arousal and sedation subscales in our sample, in comparison with Wiers et al.'s larger student sample (2005). Test-retest reliabilities and internal consistencies are satisfactory. The VAS-arousal/sedation expectancies scale consists of twelve arousal-sedation attributes. We modified the VAS-expectancies somewhat to specifically target expectations for a high dose of alcohol. Table 3 shows items belonging to each subscale. Participants indicated on a unipolar, unmarked $11 \mathrm{~cm}$ VAS scale to what extent they agreed with each of the twelve statements (e.g., "after drinking 6 or more glasses of alcohol I become energetic" disagree --_-a--agree). It was expected that VAS-arousal score would decrease as a result of the EC and VAS-sedation score would increase. 
Table 2: Test-retest reliabilities and internal consistencies of the arousal and sedation subscales of the VAS-expectancy questionnaire, in this study compared to Wiers et al. (2005)

\begin{tabular}{lccc}
\hline & $\begin{array}{c}\text { This study } \\
N=105(71 \text { controls })\end{array}$ & $\begin{array}{c}\text { Wiers et al. (2005) } \\
N=92 \text { (46 controls) }\end{array}$ \\
\hline $\begin{array}{l}\text { Test retest controls- } \\
\text { only }(r)\end{array}$ & Arousal scale & .64 & .79 \\
& Sedation scale & .70 & .53 \\
$\begin{array}{l}\text { Internal consistencies } \\
(\alpha)\end{array}$ & Arousal scale & .75 & .86 \\
& Sedation scale & .66 & .75 \\
\hline
\end{tabular}

Table 3: Items belonging to the Arousal and Sedation subscales of the VAS-expectancles questionnaire

\begin{tabular}{ll}
\hline Subscale & After drinking 6 or more glasses of alcohol I become easy \\
Arousal items & After drinking 6 or more glasses of alcohol I become active \\
& After drinking 6 or more glasses of alcohol I become funny \\
After drinking 6 or more glasses of alcohol I become excited & After drinking 6 or more glasses of alcohol I become lively \\
After drinking 6 or more glasses of alcohol I become energetic \\
After drinking 6 or more glasses of alcohol I become listless \\
After drinking 6 or more glasses of alcohol I become woozy \\
After drinking 6 or more glasses of alcohol I become quiet \\
After drinking 6 or more glasses of alcohol I become passive \\
After drinking 6 or more glasses of alcohol I become relaxed \\
After drinking 6 or more glasses of alcohol I become calm \\
After drinking 6 or more glasses of alcohol I become sleepy
\end{tabular}

Note: Items are translations of the adapted Dutch questionnaire.

\subsubsection{Procedure}

The study was conducted as a $3 \times 2 \times 2$ mixed analysis of variance (ANOVA) design (Time [pretest, 1 day post-intervention, 6 weeks post-intervention] $\times$ Condition [EC, control] $\times$ Gender [male, female]). One meeting was held in the EC condition, participants in the control condition received assessment only. Participants were recruited by two teams of two 'peers'. Each team of 'peers' consisted of a young man and a young woman. All 'peers' were university students between the ages of 19 and 21 . All peers had received a comprehensive 2-day training on the pharmacological and expectancy effects of alcohol, health risks associated with excessive use of alcohol and other important information on alcohol. In addition, 'peers' received a 1-day training in which they learned about the theoretical background of the $\mathrm{EC}$ and were able to practice in performing the EC. 'Peers' accosted young people who were spending time in front of their tent on camping sites. They were invited to participa- 
te in a study on alcohol. Approximately 35 youngsters declined the offer to participate and other young adults were then asked to take part in the study. Youngsters who agreed to join were first asked for their age. The minimum age for participation was 18 years, in accordance with the regulations for serving hard liquor that are established in the Dutch Alcohol Licensing and Catering act. Next, they were assigned to the EC or control group. Informed consent was obtained and preassessments were collected immediately (on the camping site, 1 day before the EC session). Post-assessments were done approximately 24 hours and 6 weeks after the EC session (on the camping site and by a telephone interview, respectively). Participants in the control condition were also assessed at these times. The preassessment questionnaire took approximately 10-15 minutes to complete and included the 24-hour Drinking Diary, General Drinking Questionnaire, VASexpectancies and several questions about parents' drinking, education, peer group size, length of stay and romantic relationships. Participants in the experimental group met for the EC session the next day. In total, three groups of 10-15 participants participated in the EC and each session lasted 45 to 60 minutes. All three sessions were led by a different team of 2 trained students, in accordance with a written protocol. Twenty-four hours later, peers visited the participants again to collect post-treatment measures. Approximately six weeks after the EC participants were contacted by a professional survey agency for the last assessment, which consisted of a telephone interview. For this interview the VAS-expectancies was adapted for oral administration (instead of an $11 \mathrm{~cm}$ unipolar VAS-scale, it was administered as an 11 point Likert-type scale). Furthermore, the last assessment included an oral version of the General Drinking Questionnaire.

Expectancy Challenge. The EC was based on Darkes and Goldman's Social/Sexual Challenge (Darkes \& Goldman, 1993). We adapted the EC for use in a holiday setting. This included reducing the number of EC sessions from two to one and shortening and simplifying the theoretical explanation in the EC. Participants received a name-tag and a breath analysis upon entering the room. If the breath analysis showed alcohol, they were not allowed to participate in the EC. After this, the intervention started. First, participants were asked to give five associations to the following sentence: "Alcohol makes me...". Then, approximately $50 \%$ of participants received alcohol, while the other participants received a placebo beverage. Active deception was used, all participants were told they would receive alcohol, because balanced placebo research has shown that this works better than telling people in advance that half are getting placebo (Marlatt \& Rohsenow, 1980, cf. Wiers et al., 2005). Beverages consisted of 7 oz tonic combined with 1.5 oz vodka ( 10 grams of pure alcohol) or $1.5 \mathrm{oz}$ of flat tonic. The rims of all glasses were smeared with vodka and a vodka-soaked lemon wedge was placed on the rim (active deception, Marlatt \& Rohsenow, 1980). All beverages were prepared by the 'bartender' in full view of participants. To stimulate participants' thirst, the tables had trays with salted peanuts and potato crisps on them. After the drinks were handed out, the group was split into two teams and a word game that involved drawing clues to a secret phrase (Pictionary) was played. When the game ended (after 10-15 minutes) it was revealed to participants that only half of them had consumed alcohol. Participants were 
then asked to write down who they suspected had drank alcohol (or not) and to motivate why they chose these people. The names and motivations were then disclosed on a flip-over. Identification errors were used to give information about the operation of alcohol expectancies and to start a discussion on expectancy effects of alcohol. Expectations of enhanced sociability and sexuality were specifically targeted. Lastly, participants underwent a breath analysis to measure their Blood Alcohol Concentration ( $B A C)$ and signed a form stating that they were aware of having a $B A C$ of ... \% and would bear that in mind in planning their activities and transportation the next few hours.

Assessment only. Control participants filled out questionnaires at approximately the same times as experimental participants. No intervention was given to them, nor were there any meetings held.

\subsection{Results}

\subsubsection{Initial characteristics of participants by condition}

Analyses of variance (ANOVAs) were conducted to determine if there were any differences between groups at pre-test on background characteristics, expectancies and drinking patterns. Table 4 presents baseline results for participants who completed at least the first two assessments. No significant differences were present between control and intervention group at pretest. Males drank more alcohol on a regular night out than females at pretest (21.1 and 8.2 standard drinks respectively) $F(1,115)=20.73, p<.001$, and also consumed more alcohol on a typical vacation day $(25.3$ and 12.4 standard drinks respectively), $F(1,88)=6.41, p=.01$.

\subsubsection{Feasibility of an EC in a holiday setting}

Feasibility of recruitment of participants. Participants were recruited by trained 'peers', who were able to answer any questions that emerged when filling out the pre-intervention questionnaire. It proved more difficult to recruit participants for the intervention group than for the control group. Participants in the EC group needed to come to a community centre for the 1-hour intervention. Many participants indicated that they were not willing to come to the intervention location, because it was too far away from the camping site. Moreover, many youngsters who promised to take part in the EC, failed to show up at the critical moment see Table 1). In addition, participants did not receive any compensation for the time they spent in the intervention, which may have led many people to decline the offer to participate or fail to show up at the last moment. The holiday situation may be another explanation why difficulties in recruitment were present. Young people on holiday are taking a 'time-out' from obligations, which means that appointments aren't taken as seriously and no agendas are used. Women were much more difficult to recruit for the study than men, they were less inclined to drink alcohol for the purpose of an 'alcohol experiment'. 
Table 4: Means and standard deviations for demographic and background variables

\begin{tabular}{|c|c|c|c|c|c|c|c|}
\hline \multirow[b]{2}{*}{ Variable } & \multicolumn{2}{|c|}{ EC } & \multicolumn{2}{|c|}{ Control } & \multicolumn{2}{|c|}{ Overall Sample } & \multirow[b]{2}{*}{$p$} \\
\hline & $M$ & $5 D$ & $M$ & $S D$ & $M$ & $S D$ & \\
\hline Age & 18.55 & .89 & 18.93 & 1.50 & 18.80 & 1.33 & .13 \\
\hline \multicolumn{8}{|l|}{$\begin{array}{l}\text { Alcohol consumption in } 24 \\
\text { hours before intervention, }\end{array}$} \\
\hline in glasses & 21.57 & 11.56 & 24.14 & 21.97 & 23.11 & 18.48 & .52 \\
\hline \multicolumn{8}{|l|}{ Mean alcohol consumption } \\
\hline on a night out, in glasses & 16.44 & 9.33 & 20.47 & 13.48 & 19.03 & 12.27 & .09 \\
\hline \multicolumn{8}{|l|}{ Total alcohol consumption } \\
\hline \multicolumn{8}{|l|}{ from Monday to Thursday } \\
\hline in glasses & 4.16 & 5.12 & 10.40 & 25.40 & 8.21 & 20.87 & .12 \\
\hline \multicolumn{8}{|l|}{ Alcohol expectancies- } \\
\hline Sedation mean score & 39.65 & 16.30 & 41.72 & 18.39 & 40.97 & 17.61 & .55 \\
\hline \multicolumn{8}{|l|}{ Alcohol expectancies- } \\
\hline Arousal mean score & 67.01 & 18.72 & 64.07 & 20.13 & 65.14 & 19.60 & .44 \\
\hline Group size ${ }^{1}$ & 8.02 & 3,40 & 8.31 & 4.47 & 8.20 & 4.07 & .70 \\
\hline
\end{tabular}

Note: ${ }^{1}$ Respondents reported the number of friends there were spending their holiday with (including themselves)

Feasibility of EC procedure. The EC sessions were led by a team of 2 'peers'. These 'peers' had received an 8-hour training on expectancy theory and the EC. Each 'peer' was trained by performing the EC protocol on simulation participants. However, in actual practice, participants were much more noisy and were less willing to listen to the session leaders than in the training. In some cases, some of the participants left before the end. The active deception procedure convinced participants when the protocol was followed. However, in a few cases the drinks were not served ice cold and some participants stated that they did not taste any alcohol. In those cases, little identification errors were made and 'peers' were unable to use discrepancies between predicted and actual drinkers as a starting point for their explanation. This is a point of attention for next studies. The EC was well received among young people, although some participants were a bit disappointed when they learned they had not received alcohol.

costs of the study. Most of the money was spent on salaries of 'peers' $(\$ 2100)$ and their expenses for travel and accommodation (\$1500). Because the peers also collected questionnaires, they worked more hours than they would have if no research was attached. Because the purpose of the study was to evaluate the intervention, a professional survey agency was hired for conducting telephone interviews after the holiday was over $(\$ 1400)$. Other costs were made for the purchase of vodka, tonic, lemons, posters to decorate the EC location, measuring cups, a few knives and potato crisps $(\$ 200)$.

Lessons learned. There were several points for improvement of implementation: some of the 'peers' expressed that they should have been trained more thoroughly and had missed guidance when practical problems emerged; there were not enough 'peers' present to be fully in command of the situation and the locations 
for performing the EC were too far from the camping sites, which led to high attrition by participants unwilling to show up. For the deception procedure to succeed it was important that the drinks were served chilled. It can be considered positive that intervention participants were less likely to drop out than controls. In addition, young people enjoyed participating in the intervention.

\subsubsection{Changes in Expectancies.}

VAS-expectancies were analysed in a $3 \times 2 \times 2$ (Time $\times$ Condition $\times$ Gender) mixed analysis of variance (ANOVA), with Time as within-subject variable, and Condition and Gender as between-subject variables. The VAS-arousal scale showed a statistical trend for the main effect of Time $(F(2,83)=2.99, p=.06)$. The Time $x$ Condition $x$ Gender interaction was significant $(F(2,83)=3.66, p=.03)$ therefore we analysed the interaction between Time and Condition separately in men and women. In men, the interaction between Time and Condition was not significant $(F(1,68)=1.43, p=$ .24). The expected decrease in arousal expectancies in the experimental group, was not found, although Figure 1 suggests that the EC may have worked to prevent an increase in arousal-expectancies observed in the controls. In women the Time $x$ Condition interaction showed a statistical trend $(F(1,16)=2.96, p=.08)$, but the difference was only found at pretest $(t(17)=-2.0, p=.07)$, in the absence of differences at the two posttests $\left(p^{\prime} s>.25\right)$. On the VAS-sedation subscale a statistical trend was found for Time $(F(2,83)=2.45, p=.09)$. The Time $\times$ Condition interaction was significant $(F(2,83)=3.20, p=.05)$. There was no interaction between Time, Condition and Gender $(p>.80)$. Participants in the experimental condition expected significantly more sedating effects from alcohol on the post-tests than participants in the control group. However, as shown in Figure 2, sedation expectancies did not increase in the experimental group, the significant effect came from a decrease in sedation expectancies in the control group at the six week follow-up.

\subsubsection{Changes in Alcohol Consumption}

Changes in drinking were explored on two points in time: 1. Drinking in the 24 hours after the EC and 2. Alcohol consumption on week- and weekend-days six weeks after the EC (after the vacation was over). To evaluate if a change in drinking occured in the 24 hours after the EC, a $2 \times 2 \times 2$ (Time $\times$ Condition $\times$ Gender) mixed analysis of variance (ANOVA) was conducted with alcohol consumption as a dependent variable. We compared alcohol consumption at pre-test to consumption the day after the EC. Unfortunately, many participants could not be reached for the second measurement or failed to fill out the questions about alcohol consumption, which resulted in high attrition for analyses concerning alcohol consumption (see section on selective attrition). Alcohol consumption did not change in the first 24 hours after the EC. The effect of Time was not significant $(p>.50)$ and no interaction effects were present between Time and Condition $(p>.50)$ and Time, Condition and Gender $(p>.40)$ 


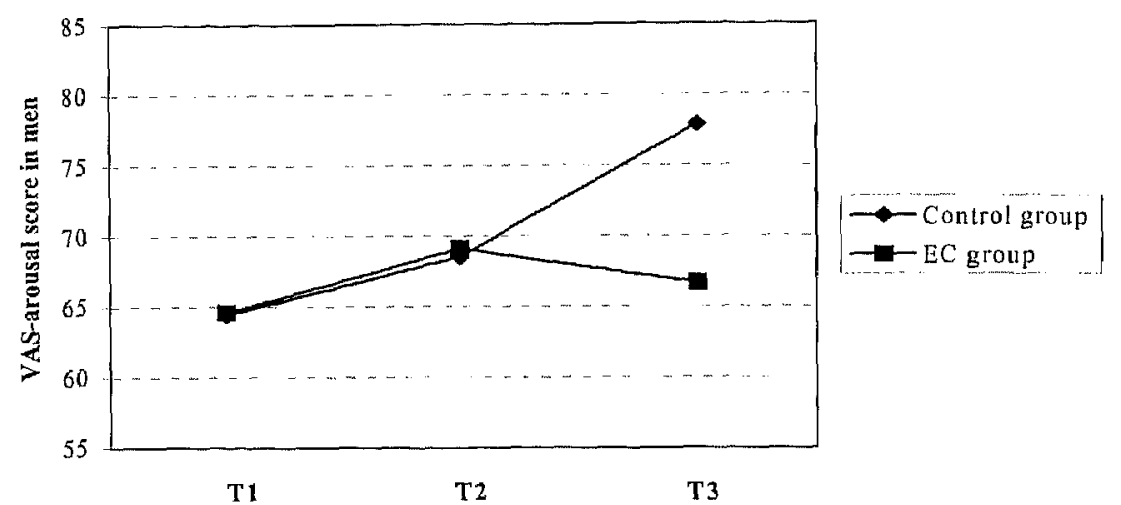

Figure 1: Change in VAS-arousal expectancies in men, by condition. Assessments were done a day before the intervention (T1), 24 hours after the intervention (T2) and six weeks after the intervention (T3).

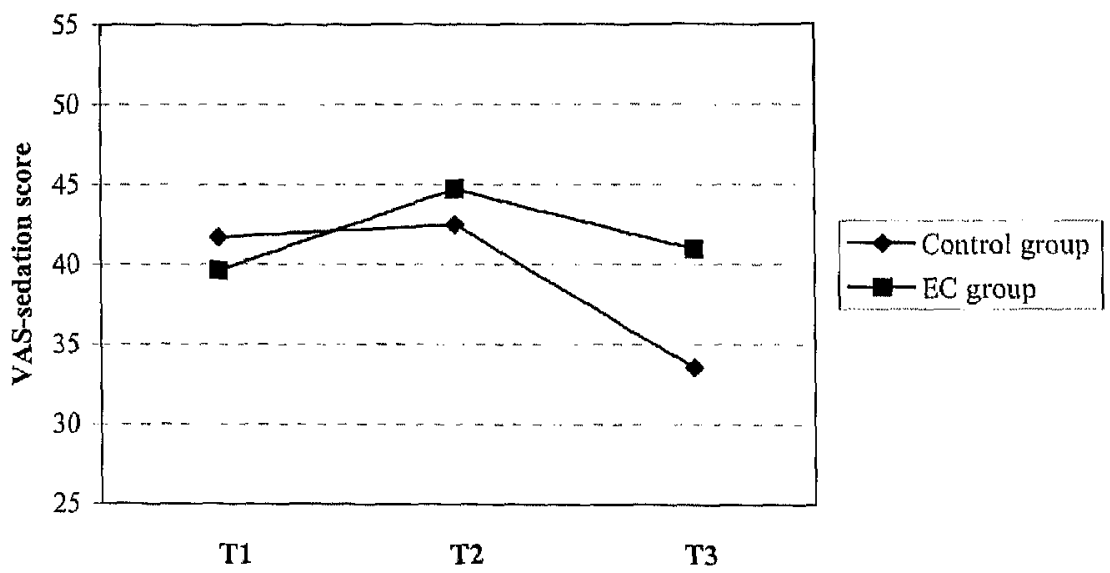

Figure 2: Change in VAS-sedation expectancies, by condition. Assessments were done a day before the intervention (T1), 24 hours after the intervention (T2) and six weeks after the intervention (T3).

Alcohol consumption six weeks after the intervention was analysed using $2 \times 2 \times 2$ (Time $\times$ Condition $\times$ Gender) mixed analyses of variance (ANOVAs) with alcohol consumption during ordinary weekdays and total consumption on a night out as dependent variables. When total alcohol consumption on a regular night out was compared from pre- to post-test a significant Time effect was found $(F(1,88)=$ $3.89, p=.05)$. Either alcohol consumption decreased in both $\mathrm{EC}$ and control participants at the post-test, or perception of alcohol consumption may have differed at home (lighter) vs. on holiday (heavier). No Time $\times$ Condition effect was present $(p>$ $.50)$ and Gender did not interact with the interaction between Time and Condition $(p>.50)$. Alcohol consumption on weekdays did not show any changes from pre- to 
post-test. Time and the interaction between Time and Condition were nonsignificant $(p>.30$ and $p>.50$ respectively). Gender did not interact with Time $x$ Condition $(p>.50)$.

We inspected relations between expectancies and alcohol consumption and unexpectedly found no correlations. Pre-test VAS-arousal and VAS-sedation expectancies did not correlate significantly with pre-test alcohol consumption on a night out, nor with pre-test alcohol consumption on holiday (Pearson's correlation VASsedation score - alcohol consumption on a night out $r=-.03$; VAS-arousal score alcohol consumption on a night out $r=.13$; VAS-sedation score - alcohol consumption on holiday $r=.11$; VAS-arousal score - alcohol consumption on holiday $r=.11$ ).

\subsection{Discussion}

This study examined whether an EC procedure that has been shown effective in (male) heavy drinkers could be transferred from a (bar-) lab setting to a targeted intervention in the field. Performing the $\mathrm{EC}$ in a real-life setting proved feasible and the EC was well-accepted by our target group. However, the current study had several limitations: high attrition, high percentage of missing values and less-than-perfect performance by the peers. Recruitment and attrition problems need to be tackled, for instance by recruiting youngsters the same day the EC takes place, performing the $E C$ at the actual camping site or providing transportation to the EC location. The 'peers' performing the EC may need to be trained more extensively and on-the-spot supervision should be present during the ECs. Presence of a third peer would aid in better performance of the EC. It is important to capture youngsters' attention by using appealing examples. Further studies should consider conducting the EC in a holiday setting with men only, since males proved easier to recruit and drank much heavier than females and may therefore decrease their drinking more after an intervention.

The intervention did not change expectancies exactly as predicted, a decrease in arousal expectancies was not found (ef. Wiers et al., 2005), nor was an increase in sedation expectancies found (both were found with the same instruments in "heavy drinking" college students in a longer EC in a bar-lab, Wiers et al., 2005). However, it is possible that the intervention countered natural changes in expectancies that may have occurred after the holiday was over. The EC may have countered the decrease in sedation expectancies that took place in the control group, but not in the intervention group.

In summary, we found some indications of effects of the EC on expectancles in "heavy drinking" men and women. Although these effects were not exactly as predicted, the EC group may have benefited from the intervention. No effects on alcohol consumption could be observed in the 24 hours after the EC, nor could a decrease be observed on week-days or during nights out at the 6-week follow-up.

In an attempt to explain our results, we inspected correlations between expectancies and alcohol use in our data. These correlations were low here (not significant) compared with previous studies (Goldman, Greenbaum \& Darkes, 1997; Leigh \& 
Stacy, 1993; Wiers et al., 1997; 2005). Five possible explanations for the low correlations between expectancies and alcohol consumption were examined. First, it is possible that expectancies were measured inaccurately. Since expectancies were measured in a holiday environment (e.g., a tent on a camping site) it is easy to conceive how error can arise. However, test-retest scores on expectancies were sufficiently good in the control group which suggests expectancies were measured reliably (see Method section). Second, we examined test-retest reliabilities of alcohol consumption on week, weekend and vacation days in the control group over time. These were satisfactory $\left(r^{\prime} s>.60\right)$, which suggests alcohol consumption was well measured. Third, within-subject variation in alcohol consumption could be high over different drinking moments due to situational influences. In our study, we found indications of the opposite, aicohol consumption during a regular night out correlated moderately with alcohol consumption on holiday $(r=.64)$, which suggests that individual characteristics determine consumption rather strongly, irrespective of the situation. Fourth, the absence of significant correlations between expectancies and alcohol use can be seen as support for criticism of the hypothesized causal role of expectancies in alcohol consumption (cf. B.T. Jones, Corbin \& Fromme, 2001). However, considering that many studies have confirmed a significant correlation between expectancies and consumption (Goldman et al., 1999), also with the instruments used here (Wiers, Van Woerden, Smulders, \& De Jong, 2002\}, we suspect specific features of the situation or target group may have caused our results to differ. Fifth, the insignificant results may be the result of "restriction of range": all included participants were heavy drinkers and maybe expectancies correlate with drinking only in mixed groups of young people (i.e. light and heavy drinkers). This is an issue for future research.

Why were only limited effects on expectancies found? First, one could argue that the questionnaire that was used to measure expectancies was inappropriate. It can not be ruled out that effects would have been found with other expectancy measures, however the VAS-expectancies questionnaire was previously successful in detecting differences in expectancies in an EC (Wiers et al., 2005) and had many advantages which made it suitable for our research design. Second, anather reason for the limited effects on expectancies may be the less-than-perfect implementation of the EC. Results of the intervention could improve after implementation flaws are tackled. Third, duration of the EC session (45-60 minutes) may have been too short for large changes to occur (the EC in Wiers et al., 2005, lasted several hours and all other effective ECs have used multiple sessions on different days, Wiers, 2002). Similarly, changing drinking behaviour may require more extensive information than the peers were able to provide in this short time. Perhaps a somewhat longer EC ( \pm 90 minutes) or an EC followed by a single session Motivational Interview (see Wiers et al., 2003; 2005) could achieve larger changes in alcohol expectancies and consumption.

In conclusion, the present study indicates that an EC with young people on holiday is feasible and generally well accepted among young people. Some effects on expectancies may have been present in men and women, however no effect on alcohol use was present. Further studies in which implementation of the intervention is optimised are needed to come to more definite conclusions about the value of the EC for interventions in binge drinking youth. 


\section{References}

Ajzen, 1. (1991). The theory of planned behavior. Organizational Behavior and Human Decision Processes, 50, $179-211$.

Corbin, W.R., McNair, L.D., \& Carter, J.A. (2001). Evaluation of a treatment-appropriate cognitive intervention for challenging alcohol outcome expectancies. Addictive Behaviors, 26, 475 - 488.

Darkes, J. \& Goldman, M. S. (1993). Expectancy challenge and drinking reduction: experimental evidence for a mediational process. Journal of Consulting and Cinical Psychology, 61, 344-353.

Darkes, J. \& Goldman, M. S. (1998). Expectancy challenge and drinking reduction: process and structure in the alcohol expectancy network. Experimental and Clinical Psychopharmacology, 6, 64-76.

Dunn, M. E., Law, H. C. \& Cruz, I. Y. (2000). Changes in activation of alcohol expectancies in memory in relation to changes in alcohol use after participation in an expectancy challenge program. Experimental and Clinical Psychopharmacology, 8, 566-575.

Goldman, M. S., Del Boca, F. K. \& Darkes, J. (1999). Alcohol expectancy theory: the application of cognitlve neuroscience. In H. T. Blane, \& K. E. Leonard (Eds.), Psychological theorles of drinking and alcoho/. ism (2nd ed.) pp. 203-246. NY: Guilford.

Goldman, M.S., Greenbaum, P.E. \& Darkes, J. (1997). A confirmatory test of hierarchical expectancy structure and predictive power discriminant validation of the alcohol expectancy questionnaire. Psychological Assessment, 9, 145-157.

Jones, B.T., Corbin, W. \& Fromme, K. (2001). A review of expectancy theory and alcohol consumption. Addiction, 91, 57-72.

Jones, L., Silvia, L. \& Richman, C.L (1995). Increased awareness and self-challenge of alcohol expectancies. Substance Abuse, 16, 77-85.

Knibbe, R.A., Oostveen, T. \& Van de Goor, I. (1991).Young people's alcohol consumption in public drinking places: reasoned behavior or related to the situation? British Journal of Addiction, 86, 1425-1433.

Larimer, M. E. \& Cronce, J. M. (2002). Identification, prevention, and treatment: a review of individualfocused strategies to reduce problematic alcohol consumption by college students. Journal of Studies on Alcohol, Supplement 14, 148-163.

Leigh, B.C. \& Stacy, A.W. (1993), Alcohol outcome expectancies: scale construction and predictive utility in higher order confirmatory factor models. Psychological Assessment, 5, 216-229.

Lemmers, L., Willems, Y., Thijssen, P., \& Osterman, C. (1998). Evaluatie zomercampagne 1997 'Lazer op met je zatte kop' [Evaluation summer campaign 1997 'Bugger off with your boozy talk']. Woerden: NIGZ.

Maddock, J.E., Wood, M.D., Davidoff, O.J., Colby, S.M. \& Monti, P.M. (1999). Alcohol expectancy challenge and alcohol use: examinatlon of a controlled trial. Paper presented at the Annual Scientific Meeting of the Research Society on Alcoholism (RSA), Santa Barbara, CA.

Marlatt, G. A. \& Rohsenow, D. J. (1980). Cognitive processes in alcohol use: expectancy and the balanced placebo design. In N. K. Mello (Ed.), Advances in substance abuse: behavioral and biological research. pp. 159-199. Greenwich: JAl Press.

Musher-Eizenman, D. R. \& Kulick, A. D. (2003). An alcohol expectancy-challenge prevention program for at-risk college women. Psychology of Addictive Behaviors, 17, 163-166.

Pos, S., Knibbe, R. \& Lemmers, L. (2001). Alle dagen feest? Een kwalitatieve studie naar omgevingsinvioe" den op het alcoholgebruik van jongeren op vakantie [Party everyday? A qualitative study of environmental influences on young people's alcohol use on holiday]. Woerden/Maastricht: NIGZ/UM.

Schwartz, N. (1999). Self-reports. How questions shape the answers. American Psychologist, 54, 93-105.

Sher, K.J., Wood, M.D., Wood, P.K. \& Raskin, G. (1996). Alcohol outcome expectancies and alcohol use: A latent variable cross-lagged panel study. Journal of Abnormal Psychology, 105, 561-574.

Van de Hoef, P., Lemmers, L. \& Knibbe, R.A. (2001). Jongeren, alcohol en vakantie: Kwantitatieve studle naar het alcoholgebruik van jongeren op vakantie in Nederland en Spanje IYoung people, alcohol, and holiday: A quantitative study on the alcohol use of youngsters on holiday in the Netherlands and Spain]. Woerden: NIGZ. 
Wiers R.W. (2002). Half full or half empty, what are we drinking? Some comments on the discussion of the causal role of aicohol expectancies as a mechanism of change. Addiction, 97, 599-600.

Wiers, R. W., Hoogeveen, K. J., Sergeant, J. A. \& Gunning, W. B. (1997). High and low dose expectancies and the differential associations with drinking in male and female adolescents and young adults, $A d-$ diction, 92, 871-888.

Wiers R. W. \& Kummeling R. C. (2004). An Experimental Test of An Alcohol Expectancy Challenge in Mixed Gender Groups of Young Heavy Drinkers. Addictive Behaviors, 29, 215-220.

Wiers R.W., Van Woerden, N., Smulders, F. T. Y. \& De Jong, P. J. (2002). Implicit and Explicit AlcoholRelated Cognitions in Heavy and Light Drinkers. Journal of Abnormal Psychology, 111, 648-658.

Wiers, R. W., Wood, M. D., Darkes, J., Corbin, W. R., Jones, B. T. \& Sher, K. J. (2003). Changing expectancies: cognitive mechanisms and context effects. Alcoholism Clinical and Experimental Research, 27, 186-197.

Wiers, R.W., Van de Luitgaarden, J.M., Van den Wildenberg, E., \& Smulders, F.T.Y. (2005). Chailenging implicit and explicit alcohol-related cognitions in young heavy drinkers. Addiction, 100(6), 806-819. 


\section{CHAPTER 5}

\section{Single-session expectancy challenge with young heavy drinkers on holiday}




\begin{abstract}
Expectancy Challenges (ECs) were used to change alcohol expectancies and alcohol consumption in young heavy drinking men (age 16-24) on holiday. The intervention took place in community centers and bars. Alcohol expectancies and consumption were assessed with paper and pencil measures prior to the intervention $(N=301)$ and two days afterwards (EC: $n=178$; controls: $n=86$ ). Six weeks after the EC, participants were interviewed by telephone (EC: $n=163$; controls: $n=71$ ). The intervention resulted in an increase in sedation expectancies in the EC group. Furthermore, the EC led to a differential reduction in alcohol consumption on a night out at the six-week posttest in the heaviest drinkers only. The reduction in alcohol consumption on a night out was not mediated by the change in sedation expectancies. These findings suggest that further research on the mechanisms of change is necessary before a single-session EC may be used in a real-life prevention setting.
\end{abstract}




\subsection{Introduction}

Many young people on holiday in seaside camping resorts in the Netherlands drink heavily. On average, young men drink 22 and young women drink 9 Dutch standard drinks per day, (Lemmers, Willems, Thijssen, \& Osterman, 1998), which, independent of type of beverage, contain 10 grams of alcohol (Knibbe \& Bloomfield, 2000). During their holiday, many drink throughout the day (Pos, Knibbe, \& Lemmers, 2001). This binge drinking episode usually lasts 1 to 2 weeks, in which several health risks are faced, varying from alcohol-related falls, traffic accidents and alcohol poisoning.

Many studies have shown correlations between expectancies and alcohol consumption (see Goldman, Del Boca \& Darkes, 1999). Positive expectancies (e.g. sociability, assertiveness, tension reduction) have been shown to predict current (Brown et al., 1985; Sher et al., 1991) and future (Christiansen et al., 1989; Stacy et al., 1990; Sher et al., 1996) alcohol consumption in a variety of age groups and drinking populations (Leigh \& Stacy, 2004). Indications exist that negative alcohol expectancies (e.g. lower self-confidence, cognitive and motor problems, negative mood) also contribute to drinking decisions (B.T. Jones \& McMahon, 1994; Leigh \& Stacy, 2004). One can also make a distinction between expectations of arousal (e.g. funny, energetic, horny) and sedation (e.g. sleepy, intoxicated, woozy; Goldman et al., 1999). Heavy drinking is associated with holding relatively strong positive-arousal expectancies, while stronger negative and sedation expectancies are associated with lower levels of drinking. On the basis of this correlation, Darkes and Goldman (1993) developed an "expectancy challenge" (EC) aimed at reducing positive arousal expectancies, which in turn should lead to a decrease in alcohol consumption. In the social Expectancy Challenge session (Darkes \& Goldman, 1993) male college students were brought together in a bar-lab, where they received a glass of vodkatonic or a placebo drink. A game (Pictionary) was played during drinking. Similarly, a week later another EC session took place, in which expectations of sexual enhancement after drinking alcohol (or placebo) were the focus of attention. In both sessions, all members were asked to write down who they thought had drunk alcohol after the games ended. Judgements were made on the basis of subjects' behaviour. Many drinkers were wrongly identified as non-drinkers and vice versa. These discrepancies were used to discuss the differences between physical and expectancy effects of alcohol. Alcohol results in interoceptive stimuli (e.g. dizziness) and molecular and motor changes (e.g. a person's posture). Drinking usually takes place in a particular context (e.g. party, disco), therefore alcohol expectancies can also appear without the pharmacological effect, that is, without drinking alcohol (Goldman, Brown, \& Christiansen, 1987). Results of the EC were compared with a traditional college prevention program and with an assessment-only group. Only the EC significantly reduced positive social and sexual expectancies and led to a decrease in alcohol consumption in heavy drinkers. The EC is one of the promising interventions with young people (Larimer \& Cronce, 2002).

Since 1993, many studies using the EC have been conducted. Some studies applied the EC to women as well (e.g. Corbin, McNair, \& Carter, 2001; Dunn, Lau, \& 
Cruz, 2000; Musher-Eizenman \& Kulick, 2003; Wiers, Van de Luitgaarden, Van den Wildenberg, \& Smulders, 2005), other studies reduced the number of sessions in the EC from two to one (e.g. Corbin et al., 2001; Wiers et al., 2005). Furthermore, some 'information-only' ECs were done, in which no actual alcohol was consumed (Corbin et al., 2001; L. Jones, Silvia, \& Richman, 1995). In general, ECs succeeded in reducing alcohol consumption in male college students (Darkes \& Goldman, 1993, 1998; Wiers et al., 2005). In women, reductions in positive alcohol expectancies were demonstrated, but no differential reductions in alcohol use were achieved (e.g. Wiers et al., 2005). Multi-session ECs may result in larger reductions in positivearousal expectancies and alcohol consumption than single-session protocols (Darkes \& Goldman, 1993, 1998; Wiers, 2002), however single-session ECs are easier conducted as an intervention in real-life settings and resulted in equally large effects (at least in one study, Wiers et al., 2005).

In 2002, we conducted a first pilot study in which the feasibility of a singlesession EC with youth on holiday was shown (Van de Luitgaarden, Wiers, Knibbe, \& Boon, 2006). This was the first EC conducted in a real-life setting. In this study we used an optimized version of this EC. Only male participants between the ages of 16 and 24 were included, because previous experience had taught us women are much more difficult to recruit and drink much less on average (Van de Luitgaarden et al., 2006). Participants were recruited in seaside camping resorts in the Netherlands. We hypothesized a decrease in positive-arousal expectancies as a result of the EC, and an increase in negative-sedation expectancies. Furthermore, we investigated whether a change in expectancies had caused a change in alcohol consumption (mediation analysis). Partial mediation was previously demonstrated by Wiers et al. (2005), in other EC studies mediation was not formally tested (Jones et al., 2001; Wiers, 2002).

\subsection{Methods}

\subsubsection{Participants}

Young men ( $N=301$, mean age 17.8 years; minimum age 16.0 years; the minimum legal drinking age) spending a vacation on one of two seaside vacation destinations were recruited by trained students ('peers'). Most participants $(96.0 \%)$ were on holiday with friends, without their parents or partner. Participants reported drinking on average 23.8 Dutch standard-drinks per day during vacation and 32.1 standarddrinks in a regular week when not on vacation. Young men in our study are very heavy drinkers compared to the average consumption of Dutch young men aged 16 to 24 (15.5 standard drinks per week; Van Dijck \& Knibbe, 2005), and compared to recommendations (2-3 drinks max. per day, at least two alcohol-free days a week).

The study was presented somewhat differently to participants in the EC and control group. 'Peers' approached potential EC participants $(n=186)$ at camping sites on the Isle of Terschelling. Young men were asked if they would like to participate in a study on alcohol, which would involve drinking 2 bottles of Smirnoff Black 
ice (a popular premix). They were instructed to be sober for the meeting. They were told that they would receive $€ 7.5$ after completing the second measurement two days later and that they would be interviewed by telephoned two months later. Young men spending a vacation at the Isle of Texel constituted the control group ( $n$ $=115$ ). Controls were invited to participate in a study on alcohol, which would involve filling out questionnaires twice and being interviewed over the telephone. They received $€ 5$ for participation, somewhat less than EC participants because of a smaller time-investment. A quasi-experimental design was chosen to ensure that a sufficient number of participants could be recruited within the given space of time, and had the additional advantage that experimental and control subjects were unlikely to come into contact with each other.

Attrition. Two days after the initial assessment, 37 participants dropped out because the 'peers' were unable to contact them for filling out further questionnaires. Six weeks after the EC, an additional 30 participants could not be reached for the telephone interview (see Table 1).

To examine whether participants who did not complete all measurements differed from those who did, logistic regression analyses ${ }^{1}$ were conducted for dropout two days and 6 weeks after the initial assessment. Significantly more control participants dropped out after two days $(p<.001)$. In addition, both age and baseline vacation consumption showed a positive association with dropout at that point in time ( $p<.05$ and $p<.01$, respectively). Other variables did not predict attrition after two days. Attrition six weeks after the EC could not be predicted from any of the variables.

Table 1: Design of the study and questionnaires used

\begin{tabular}{lllll}
\hline & TO & T1 & T2 & T3 \\
& Pretest & Directly after EC & +2 days & + 6 weeks \\
& EC: $n=186$ & EC: $n=185$ & EC: $n=178$ & EC: $n=163$ \\
Control: $n=115$ & & Control: $n=86$ & Control: $n=71$ \\
\cline { 2 - 4 } & 24-hour Drinking & VAS-Expectancles & 24-hour Drinking & Drinking \\
& Diary & Questionnaire & Diary & Questionnaire \\
& Drinking & & VAS-Expectancies & VAS-Expectancies \\
& Questionnaire & & Questionnalre & Questionnaire \\
& VAS-Expectancies & & & \\
Questionnaire & & & \\
Demographics & & & \\
\hline
\end{tabular}

1 Results were checked by multi-level logistic regression analyses in which attrition was analysed taking into account possible data dependencies due to participants receiving treatment in subgroups. There was a lack of variability at the level of the subgroup in the EC condition, thus confirming the results of the initial logistic regression analyses, in which the effects of nesting in subgroups were disregarded. 


\subsubsection{Procedures}

Design. The study was conducted as a repeated measures design over an approximate 2-month time interval with one intervention session held in the EC group. The control group received assessment only. Table 1 outlines the design of the study and details the questionnaires used. Trained 'peers' led all EC sessions. In total, 16 ECs were conducted with 10-15 young men participating in each session. The team of peers consisted of 4 female students and 1 male student. Three varying members of the team conducted each EC. Session leadership changed from session to session. A graduate student supervised all ECs. All components of the EC were covered in every EC session. The EC was a modified version of the procedure used by Darkes and Goldman (1993). Changes in the protocol included telling participants everyone would receive alcohol (active deception, Marlatt \& Rohsenow, 1980, as in Wiers et al., 2005\}, using only one session instead of two and omitting the game in which attractiveness of pictures of women was rated. Furthermore, we used a premix instead of vodka-tonic, because the Dutch Alcohol Licensing and Catering act forbids serving vodka-tonic to persons younger than 18 years, while a premix can be served to persons of 16 years and older.

Expectancy challenge. Participants received a nametag and were breathilized upon entering. They then filled out informed consent and the baseline questionnaire. The actual intervention started with asking the participants to give associations to the sentence "Alcohol makes me...". The session leader wrote all associations on a flip-over. Then, half of all participants received two alcoholic drinks, while the other half received placebo beverages. The placebo beverage was a low-alcoholic mix of $140 \mathrm{cc}$ Spa Clear Citrus, $80 \mathrm{cc}$ tonic and $55 \mathrm{cc}$ Smirnoff Black Ice. The placebo drink contained $1 \%$ alcohol ( 3 grams of alcohol per bottle). The alcoholic mixed drink consisted of $240 \mathrm{cc}$ Smirnoff Black Ice and $35 \mathrm{cc}$ tonic and contained $4 \%$ alcohol ( \pm 11 grams of alcohol per bottle). Tonic was added to the alcoholic Smirnoff Black Ice drink to make the taste of the alcoholic and placebo drink more alike. All participants received two refilled Smirnoff Black ice bottles of $275 \mathrm{ml}$ each. Half received two bottles containing alcohol; the other half two placebo bottles. Half of the participants received bottles with straws, independent of the contents of the bottle. This was done to divert attention away from the taste of the drinks. Many participants presumed the straws were the experiment, and therefore did not focus on the taste of the drinks. The tables had trays with salted peanuts and potato crisps on them to stimulate participants' thirst. After the drinks were handed out, the group was divided in two teams. Participants engaged in a game of Pictionary, in which team members graphically illustrate clues on a flip-over in an attempt to communicate a secret phrase to other team members. Sexually charged secret phrases were used, which were a useful leg up to the sexual alcohol expectancies discussed later. After the game was completed ( 15 minutes), it was disclosed that only half of all subjects had received (about) two standard drinks of alcohol, while the other half had received almost no alcohol. Participants were asked to list the drinkers and to motivate their choice. In every EC, several misclassifications were made. Identification errors were used to discuss alcohol expectancies. The main message was that expectancies are often the cause of behavioural effects after drinking, instead of pharmacological 
oroperties of alcohol. Special attention was given to social and sexual alcohol expectancies. The information was closely modelled after the original protocol. After the EC, participants filled out another questionnaire (see Table 1). Furthermore, appointments were made to fill out questionnaires at the campsite two days later. Participants were breathalysed signed a form stating that they were aware of their alcohol-level and would be cautious in choosing their activities and transportation the next few hours.

Assessment-only. Control participants filled out an informed consent form and baseline measures at the camping site immediately after they agreed to participate in the study. No group meetings were held, questionnaires were filled out individually. Appointments were made to collect measures two days later.

\subsubsection{Measures}

Demographics Questionnaire. Background characteristics of participants were measured with a self devised demographics questionnaire. Questions addressed age, housing situation, romantic relationships, job, peer group size and composition, length of stay, income and education.

Visual Analogue Scale (VAS) arousal-sedation expectancies. Alcohol expectancies were measured by the VAS-expectancies questionnaire (Wiers, Van Woerden, Smulders, \& De Jong, 2002; Wiers et al., 2005). We used a modified version in which expectations for a high dose of alcohol were specifically targeted (see Van de Luitgaarden et al., 2006), because these are the best predictor of alcohol use in heavy drinking young males (Wiers, Hoogeveen, Sergeant, \& Gunning, 1997). The VASarousal/sedation expectancies scale consists of twelve arousal-sedation attributes. Participants indicated on a 7-point VAS scale to what extent they agreed with each of twelve statements (e.g., "after drinking 6 or more glasses of alcohol I become excited"). The VAS-expectancies questionnaire was most suitable for our study because it is relatively short, includes equal numbers of items describing desirable and undesirable effects (see Leigh \& Stacy, 1993) and reduces memory-effects that can occur with repeated administrations (Schwartz, 1999). Furthermore, it was previously successful in detecting changes in expectancies as a result of an EC procedure (Wiers et al., 2005) and in predicting actual behaviour under the influence of alcohol (Bot, Engels, \& Knibbe, 2005). Internal consistencies as well as test-retest reliabilities of this questionnaire are satisfactory (Van de Luitgaarden et al., 2006; Wiers et al., 2005). In the present study, internal consistencies (Cronbach's $\alpha$ ) of the arousal and sedation subscales were .72 and .67 , respectively. Test-retest reliabilities (controls-only) were .57 for the sedation subscale and .74 for the arousal subscale.

Drinking Questionnaire. Alcohol consumption before and after the vacation was measured by questions about drinking behaviour on typical week-days, frequency of going out in the weekend, number of glasses consumed while going out and number of glasses consumed prior to going out (at home or at a friend's house).

Drinking Diary. At baseline, participants were asked if they had drank any alcohol the previous day, and if the answer was 'yes', if they had drank alcohol in the 
morning, in the afternoon, in the evening or at night. They were also asked to report where the alcohol was consumed (e.g. pub / disco, camping site, beach) and how much standard glasses of alcohol they drank in each location at each time of day. Two days after the EC, both control and EC participants filled out the Drinking Diary over the previous day.

\subsubsection{Analyses}

Effects of the EC on scores on the VAS-expectancies questionnaire were analyzed with multiple regression analyses. Expectancies two days after the EC (short-term) and expectancies 6-8 weeks after the EC (long-term) were dependent variables. Condition was the independent variable, and covariates were included (age, money, number of friends with whom one spends the holiday, education, baseline alcohol consumption on a night out and during ordinary week and weekend days, as well as baseline arousal and sedation expectancies). Inter-correlations between independent variables were all below .70 , excluding collinearity problems. Paired t-tests were performed to investigate if expectancies had changed immediately after the intervention in the EC group. It was hypothesized that positive-arousal expectancies should decrease and negative-sedation expectancies increase as a result of the EC. Furthermore, we tested the effect of the intervention on alcohol consumption. Alcohol consumption on holiday two days after the EC, and alcohol use on a regular night out 6-8 weeks after the EC were dependent variables ${ }^{2}$ (in separate analyses). Again, condition was an independent variable, and the fore mentioned covariates were included (baseline holiday consumption was included as an additional covariate in the analysis of consumption on holiday two days after the intervention). We hypothesized a reduction in alcohol consumption in the EC group. Previous studies indicated that the impact of the intervention may be related to participants' level of baseline consumption (Darkes \& Goldman, 1998), therefore we tested interactions between baseline drinking level and condition.

EC participants were nested within subgroups of 10-15 young men who received the intervention at the same time, therefore control multi-level analyses were performed (with MlwiN version 2.02), which tested the effects of the intervention taking into account possible data dependencies within subgroups. All analyses cemonstrated a lack of random variability at the level of the subgroup in the EC condition, thus confirming the results of the multiple regression analyses, in which the effects of nesting in subgroups were disregarded. Finally, mediation analyses (Baron \& Kenny, 1986) were performed using AMOS 5.0 structural equation modelling (SEM) software.

\footnotetext{
2 Indices of alcohol consumption showed deviances from normality. Therefore, all were log transformed with the following formula: InAlcUse $=$ in (AlcUse +1 ), where one is added to make the inclusion of nondrinkers possible. Transformations may make interpretation of results more difficult (Stevens, 1992), therefore non-transformed indices of alcohol use are reported here. However, all results were confirmed with log-transformed alcohol variables, and the pattern of results was the same.
} 


\subsection{Results}

\subsubsection{Baseline Drinking and Expectancies}

Table 2 presents demographic and background variables of our sample. EC participants were significantly older than controls at baseline $F(1,299)=4.44, p<.05$. Age, money, number of friends with whom one spends the holiday, education, baseline alcohol consumption on a night out and during ordinary week and weekend days, as well as baseline arousal and sedation expectancies were included as covariates in further analyses.

Table 2: Means and standard deviations by condition for demographic and background variables

\begin{tabular}{|c|c|c|c|c|c|c|c|}
\hline \multirow[b]{2}{*}{ Variable } & \multicolumn{2}{|c|}{$\begin{array}{c}\text { EC } \\
\langle n=186)\end{array}$} & \multicolumn{2}{|c|}{$\begin{array}{l}\text { Control } \\
(n=115)\end{array}$} & \multicolumn{2}{|c|}{$\begin{array}{l}\text { Overall Sample } \\
\qquad(N=301)\end{array}$} & \multirow[b]{2}{*}{$p$} \\
\hline & $M$ & $S D$ & $M$ & $5 D$ & $M$ & $S D$ & \\
\hline Age, in years & 17.98 & 1.54 & 17.57 & 1.79 & 17.83 & 1.65 & .04 \\
\hline $\begin{array}{l}\text { Alcohol consumption in } 24 \\
\text { hours before intervention, in } \\
\text { Dutch standard glasses }\end{array}$ & 24.27 & 13.14 & 22.35 & 11.83 & 23.55 & 12.68 & ns \\
\hline $\begin{array}{l}\text { Mean alcohol consumption } \\
\text { on a night out, in Dutch } \\
\text { standard glasses }\end{array}$ & 14.98 & 6.44 & 15.29 & 8.98 & 15.10 & 7.50 & ns \\
\hline $\begin{array}{l}\text { Amount of money to spend } \\
\text { per month, in Euros }\end{array}$ & 296.43 & 233,95 & 246.36 & 239.39 & 277.01 & 236.93 & ns \\
\hline Education level" & 2.00 & $\cdots$ & 3.00 & $-\cdots$ & 2.00 & $--\infty$ & ns \\
\hline $\begin{array}{l}\text { Size of circle of friends on } \\
\text { holiday (excl. themselves) }\end{array}$ & 6.20 & 4.37 & 6.34 & 4.99 & 6.25 & 4.61 & ns \\
\hline $\begin{array}{l}\text { Total alcohol consumption } \\
\text { from Friday to Sunday in } \\
\text { Dutch Standard glasses }\end{array}$ & 26.10 & 14.47 & 25.55 & 19.06 & 25.87 & 16.32 & ns \\
\hline $\begin{array}{l}\text { Total alcohol consumption } \\
\text { from Monday to Thursday in } \\
\text { Dutch standard glasses }\end{array}$ & 6.33 & 7.87 & 8.03 & 11.22 & 6.97 & 9.29 & ns \\
\hline $\begin{array}{l}\text { Alcohol expectancies- } \\
\text { Arousal sumscore" }\end{array}$ & 31.13 & 7.43 & 31.29 & 8.66 & 31.19 & 7.91 & ns \\
\hline $\begin{array}{l}\text { Alcohol expectancies- } \\
\text { Sedation sumscore }\end{array}$ & 21.61 & 6.52 & 20.30 & 6.38 & 21.11 & 6.49 & ns \\
\hline
\end{tabular}

Note. "Mode is presented instead of mean, education was coded as $1=$ low education level, $2=$ moderate education level, 3 = high education level. "Arousal expectancles were computed as the sum of 7 items measuring arousal expectancies on a 7 point Likert scale. "Seclation expectancles were computed as the sum of 7 items measuring sedation expectancles on a 7 point Likert scale. 


\subsubsection{Effects of the EC on expectancies}

Effects on sedation expectancies. Sedation expectancies of EC participants had increased immediately after the intervention $t(182)=-4.34, p<.001$. Condition significantly predicted VAS-sedation expectancies two days after the EC, with sedation expectancies being significantly higher in the intervention group compared to the control group $F(1,259)=12.38, p=.001$. Six weeks after the $E C$, condition was still a significant predictor of sedation expectancies, $F(1,217)=9.75, p<.01$ (see Figure 1, T2 and T3).

Effects on arousal expectancies. Arousal expectancies of EC participants had not changed immediately after the intervention $(p>40)$. Condition did not predict VASarousal expectancies two days after the EC $(p>.50)$, nor did it predict VAS-arousal expectancies six weeks later $(p>.50)$.

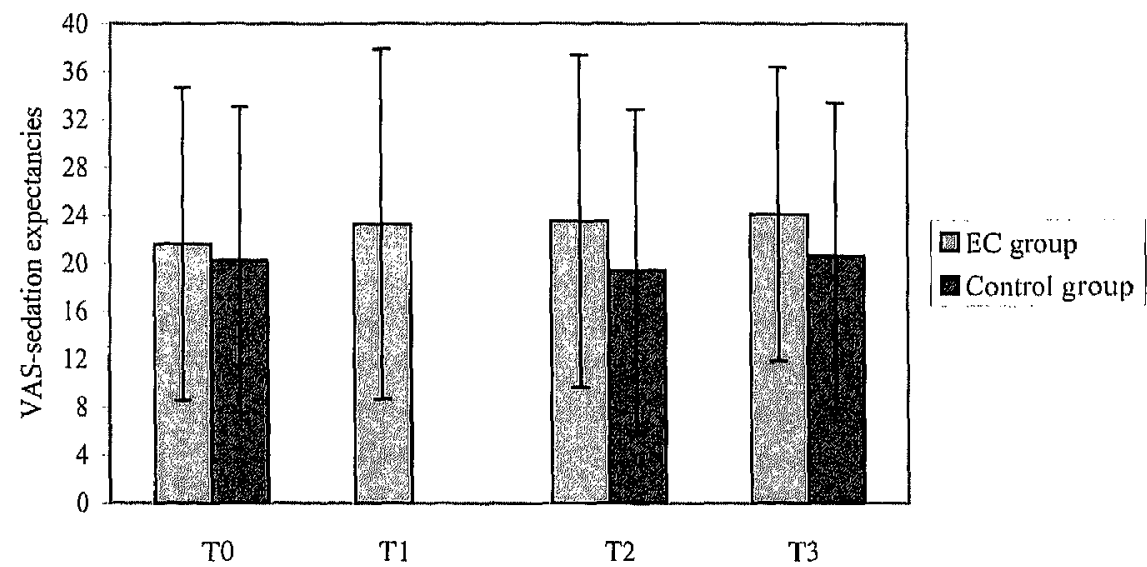

Figure 1: VAS-sedation expectancles, by condition. Assessments were conducted prior to the intervention (TO), immediately after the intervention (only in the EC group, T1) , 2 days after the intervention (T2) and six weeks after the intervention (T3). Y error bars depict $95 \%$ confidence intervals

\subsubsection{Effects of the EC on alcohol consumption}

Alcohol consumption on holiday. The interaction between alcohol consumption on holiday at baseline and condition was non-significant $(p>.50)$. The regression analysis showed no effect of condition on alcohol consumption on vacation, two days after the intervention $(p>.50)$

Alcohol consumption on a regular night out. The interaction between alcohol consumption on a regular night out at baseline and condition was significant $F$ ( 1 , $231)=5.48, p<.05$. Therefore, we performed a median split on baseline consumption levels of the entire sample. Participants who drank 14 standard drinks or less on a 'regular night out' constituted the lower-level drinking group, while partici- 
pants who drank more than 14 drinks constituted the heaviest drinking group. The regression analyses were done separately for the lower-level drinking group and the heaviest drinking group. Condition was an independent variable, and age, money, number of friends with whom one spends the holiday, education, baseline alcohol consumption on a night out and during ordinary week and weekend days, as well as baseline arousal and sedation expectancies were included as covariates. The intervention resulted in a stronger decrease in alcohol consumption on a regular night out in the heaviest drinking EC group compared to the heaviest drinking control group $F(1,96)=7.91, p=.01$ (see Figure 2 ). The EC did not result in changes in the lower-level drinking group $(p>.50)$.

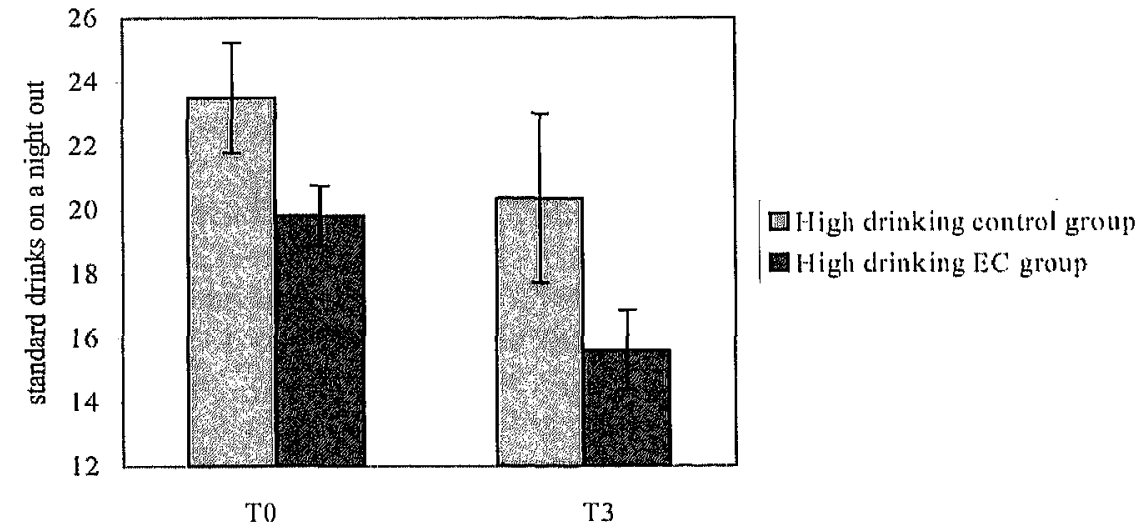

Figure 2: Alcohol consumption on a regular night out, in the heavlest drinking group, per condition. TO = pretest, $T 3=$ six weeks after the intervention. Y error bars depict $95 \%$ confidence intervals.

\subsubsection{Correlations between expectancies and alcohol consumption}

We examined correlations between baseline VAS-arousal and sedation expectancies on the one hand, and total alcohol use during week- and weekend days, on a night out, and during vacation on the other hand. VAS-sedation expectancies correlated negatively with alcohol consumption on a night out $(r=-.152, p<.01)$, with total consumption from Monday through Friday $(r=-.121, p<.05)$ and with consumption during the weekend $(r=-.137, p<.05)$. Sedation expectancies did not correlate with alcohol use on holiday. All correlations between arousal expectancies and consumption were non-significant ( $p$ 's $>.40$ ). Furthermore, alcohol use on holiday correlated positively with consumption on a regular night out $(r=.622, p<.001)$ and with total weekly alcohol consumption when not on vacation $(r=.552, p<.001)$. 


\subsubsection{Mediation analyses}

Mediation was tested in the heaviest dirinkers for alcohol consumption on a regular night out 6 weeks after the intervention, using a model specifically proposed for the present design (MacKinnon, 1994; cf. Wiers et al., 2005). Lower-level drinkers were excluded from the analysis because they cannot be expected to show any mediation (no change in drinking). In the present study both the mediator 'sedation expectancies', as well as the outcome variable 'alcohol consumption on a night out' (in the heaviest drinkers of the EC group) were significantly changed by the intervention. The direct pathway from condition to alcohol consumption on a night out during follow-up was significant $(p=.05)$, as well as the direct pathway from condition to post-test sedation expectancies $(p<.05)$. However, the pathway from post-test sedation expectancies to alcohol consumption at follow-up was non-significant, indicating absence of mediation (Fig. 3a). To test for the possibility that the inclusion of the control group suppressed a mediation effect (Shrout \& Bolger, 2002), a mediation analysis was performed in which controls were excluded (Fig. 3b). However, the direct pathway from sedation expectancies two days after the EC to alcohol consumption on a night out at follow-up remained non-significant $(p>.40)$, as well as the pathway from baseline sedation expectancies to alcohol consumption on a night out at follow-up $(p>10)$. Hence, the EC-induced change in sedation expectancies was not a mediator of the reduction in alcohol consumption in the heaviest drinkers in the EC group during a night out six weeks later (Fig. 3).

\subsection{Discussion}

The findings of our study partly replicate findings from other studies (Darkes \& Goldman, 1993, 1998; Dunn et al., 2000; Wiers \& Kummeling, 2004; Wiers et al., 2005). Participants in the EC condition increased their sedation expectancies after the $E C$, and the heaviest drinking EC participants reduced their alcohol consumption on a regular night out more than control participants. This differential reduction in consumption was small, but significant. The effect size was much smaller $(d=0.12)$ than in previous EC studies, in which medium to large effect sizes were reported (Darkes \& Goldman, 1993; Wiers et al., 2005). Furthermore, no mediation of alcohol use by sedation expectancies took place. This is somewhat surprising, because the idea behind the $\mathrm{EC}$ is to decrease consumption by changing expectancies. There are several possible explanations for our null finding regarding mediation. First, the test-retest reliabilities of the sedation subscale were fairly low in our sample, while other studies found adequate reliability (Wiers et al., 2005; Van de Luitgaarden et al., 2006). Perhaps, alcohol expectancies in youth on holiday are not stable, but vary over time, causing them to be less predictive of alcohol use in this population. Second, our null finding may add support to questions about the mechanism of change evoked by the EC (cf. B.T. Jones, Corbin \& Fromme, 2001). As other authors have noted, expectancy change is not the sole means whereby drinking might change (Del Boca \& Darkes, 2001; B.T. Jones et al., 2001; Wiers et al., 2005). It may be that 


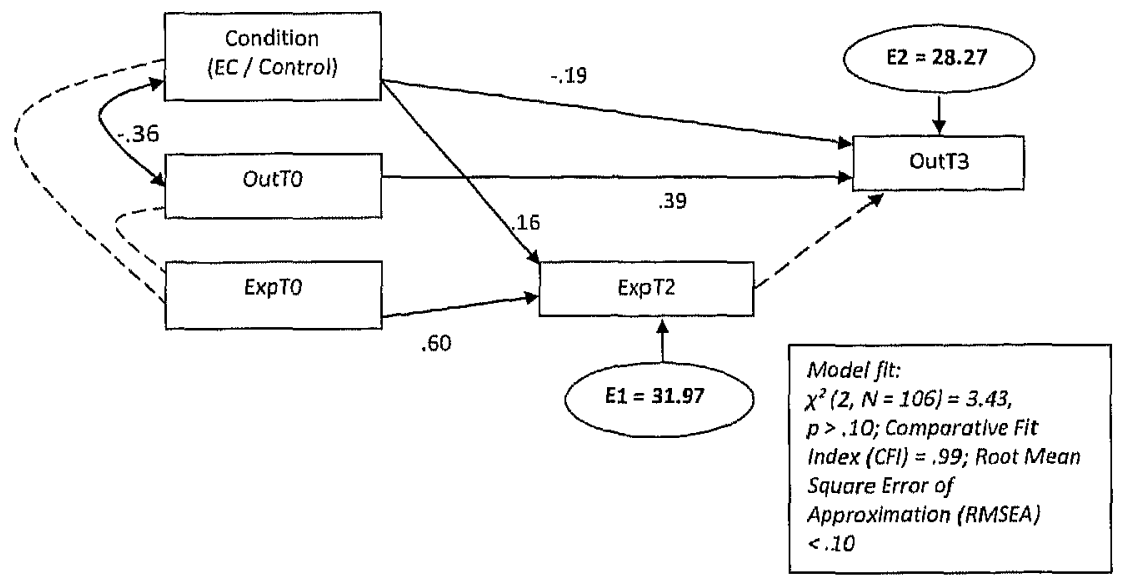

Figure 3a: Mediation model of alcohol use on a regular night out at 6-week follow-up (heaviest drinkers only; Standardized Bootstrap Estimates)

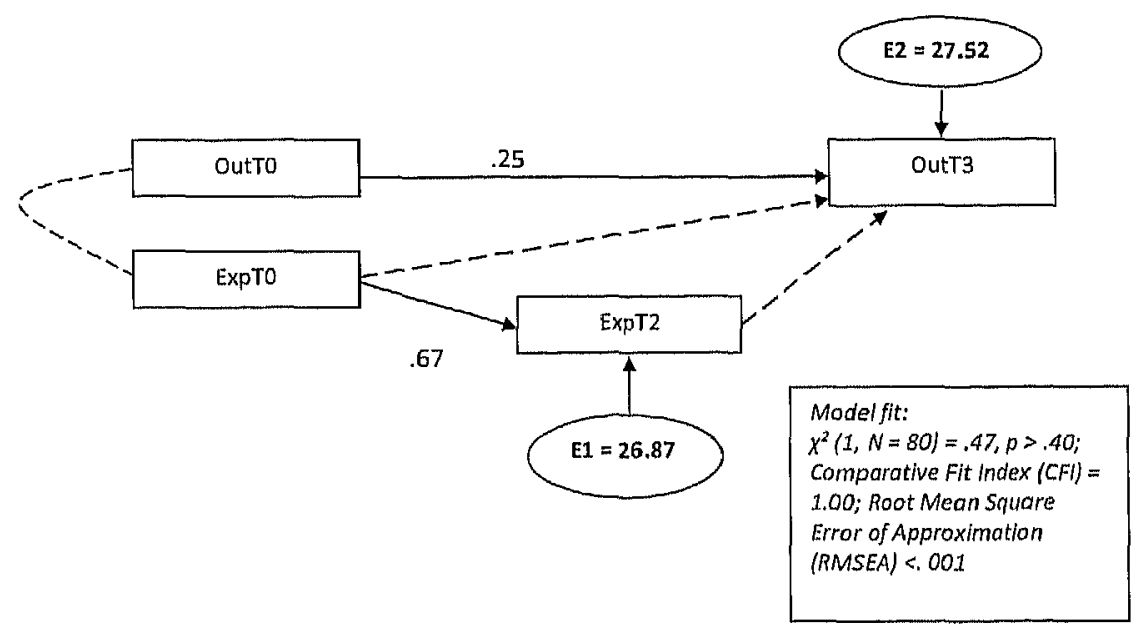

Figure 3b: Mediation model of alcohol use on a regular night out at 6-week follow-up (heavlest drinking EC participants only; Standardized Bootstrap Estimates)

Figure 3: Mediation analysis of alcohol consumption on a night out 6 weeks after the intervention in the heaviest drinkers. It was tested if the increase in sedation expectancies after the EC medlated the prospective reduction in alcohol use on a night out in the heaviest drinkers six weeks later (3a). Additionally, mediation was tested in the heaviest drinking EC participants only (3b). Drawn lines depict significant paths, dashed lines non-significant paths. Bias-corrected maximum likelihood bootstrap estimates of standardized regression weights are presented for significant paths. The bias-corrected bootstrap method has the best power to detect mediation effects (Mackinnon, Lockwood, \& Williams, 2004). Out $=$ alcohol use on a night out in standard drinks; Exp $=$ sedation expectancies; $E=$ estimated error variance; $\mathrm{T} 0=$ pretest, $\mathrm{T} 2 \mathrm{=}$ two days after the intervention, $\mathrm{T} 3=\mathrm{six}$ weeks after the intervention. 
other characteristics of the intervention caused the small change in heavy drinkers (e.g. seeing one's breath alcohol level after drinking). Arousal expectancies did not change after the intervention. To explain this, one could argue that the VASexpectancies questionnaire was inappropriate. Although the test-retest reliabilities of the sedation subscale were indeed rather low in our sample, test-retest reliabilities of the arousal subscale were actually sufficient. It is possible that the intervention simply did not succeed in changing arousal expectancies, perhaps because there was only one session per group and the duration of each session was only 1.5 to 2 hours. Similarly, more sessions or a longer duration of the EC may be necessary to change total alcohol consumption in the weekend and consumption from Monday through Friday. It may also be the case that these young men simply drink too heavily for this intervention.

In our previous EC study with youth on holiday, no correlations were found between baseline VAS-expectancies and baseline alcohol consumption on a night out and on holiday (Van de Luitgaarden et al., 2006). In the present study, we did find a significant correlation between baseline VAS-sedation expectancies and consumption on a regular night out. This is in line with other studies showing correlations between negative-sedation alcohol expectancies and consumption (see Goldman et al., 1999). However, sedation expectancies did not correlate with alcohol use on holiday, which suggests that drinking behaviour on holiday may be less influenced by these expectancies than alcohol consumption outside the vacation. VASarousal expectancies did not correlate with measures of alcohol consumption. We found this in our previous study as well (Van de Luitgaarden et al., 2006), while other studies did find significant correlations between arousal expectancies and consumption with the same VAS-expectancies questionnaire in samples of heavy and light drinkers (Wiers et al., 2002). Possibly, arousal expectancies predict alcohol consumption in beginning drinkers, but lose their predictive value when alcohol use exceeds a certain threshold (in accordance with Dichiara's incentive habit theory, cf. Mogg, Field, \& Bradley, 2005). In our study, almost all participants were very heavy drinkers while on holiday. In daily life, they drank approximately the same amount of alcohol as participants in another study (Wiers et al. 2005), which did demonstrate significant correlations between arousal expectancies and alcohol consumption. A related explanation for the lack of correlation could be that arousal expectancies lose their predictive value when they exceed a certain threshold. Nearly all participants in our study had high arousal expectancies.

The present study is one of the largest studies on the effects of the EC. It is also one of the first studies in which the EC was applied to heavy drinkers in a natural setting. A limitation of the present study is the quasi-experimental design. In the analyses, we corrected for differences in background characteristics, however it cannot be completely ruled out that other differences between EC and control group may have been present. Performing an EC with young people on holiday was feasible (Van de Luitgaarden et al., 2006) and a significant increase in sedation expectancies was achieved. The EC caused a small differential reduction in consumption on a regular night out among the heaviest drinkers, but major reductions in alcohol use among lower-level drinkers and mediation of alcohol use by expectancies 
are lacking. Many other single-session ECs found limited results (e.g. Corbin et al., 2001, Van de Luitgaarden et al., 2006). Multiple session ECs may achieve more results, but are too difficult to use with young people on holiday, since the population changes rapidly and no agendas are kept.

It may be that the EC is not particularly suited for application with young people on holiday. The costs of performing the EC are high (see Van de Luitgaarden et al., 2006), relatively few people can be reached, and the effects on consumption have been relatively small. Furthermore, the lack of mediation in our sample suggests that the 'active ingredients' in this EC are not known, which makes application in practice somewhat problematic at this point in time. Future studies should establish if changes in consumption after the EC are caused by changes in expectancies (B.T. Jones et al., 2001). If mediation is confirmed, the EC might be useful in the context of social work, performed with youth at risk in a community centre. In such a situation, multiple EC sessions could be performed because the social worker has an ongoing relationship with these young people. Additionally, it would be easier to provide evidence for the efficacy of the procedure, because there would be more potential for follow-up measurements. Another possible use for the EC would be as a fun, accepted leg up to personal follow-up contact (e.g. Motivational Interviewing). Future studies are needed to evaluate the potential of the EC as a practiceapplied intervention. 


\section{References}

Baron, R.M. \& Kenny, D.A. (1986). The moderator-mediator variable distinction in social psychological research: conceptual, strategic, and statistical considerations. Journal of Personality and Social Psychology, 51, $1173-1182$.

Bot, S.M., Engels, R.C. \& Knibbe, R.A. (2005). The effects of alcohol expectancies on drinking behaviour in peer groups: observations in a naturalistic setting. Addiction, 100, 1270-1279.

Corbin, W.R., McNair, L.D. \& Carter, J.A. (2001). Evaluation of a treatment-appropriate cognitive intervention for challenging alcohol outcome expectancies. Addictive Behaviors, 26, $475-488$.

Darkes, J. \& Goldman, M. S. (1993). Expectancy challenge and drinking reduction: experimental evidence for a mediational process. Journal of Consulting and Clinical Psychology, 61, 344-353.

Darkes, J. \& Goldman, M. S. (1998). Expectancy challenge and drinking reduction: process and structure in the alcohol expectancy network. Experimental and Clinical Psychopharmacology, 6, 64-76.

Del Boca, F.K. \& Darkes, J. (2001). Is the glass half full or half empty? An evaluation of the status of expectancies as causal agents. Addiction, 96, 1681-1683.

Dunn, M. E., Lau, H. C. \& Cruz, I. Y. (2000). Changes in activation of alcohol expectancies in memory in relation to changes in alcohol use after participation in an expectancy challenge program. Experimental and Clinical Psychopharmacology, 8, 566-575.

Goldman, M. S., Del Boca, F. K. \& Darkes, J. (1999). Alcohol expectancy theory: the application of cognitive neuroscience. In H. T. Blane, \& K. E. Leonard (Eds.), Psychological theories of drinking and alcoholism (2nd ed.). pp. 203-246. NY: Guilford.

Jones, B.T., Corbin, W. \& Fromme, K. (2001). A review of expectancy theory and alcohol consumption. Addiction, 91, 57-72.

Jones, L., Silvia, L. \& Richman, C.L (1995). Increased awareness and self-challenge of alcohol expectancies. Substance Abuse, 16, 77-85.

Knibbe, R.A. \& Bloomfield, K. (2000). Alcohol consumption estimates in surveys in Europe: comparability and sensitivity for gender differences. Substance Abuse, 22, 23-38.

Larimer, M. E. \& Cronce, J. M. (2002). Identification, prevention, and treatment: a review of individualfocused strategies to reduce problematic alcohol consumption by college students. Journal of Studies on Alcohol, Supplement 14, 148-163.

Leigh, B.C. \& Stacy, A.W. (1993). Alcohol outcome expectancies: scale construction and predictlve utllity in higher order confirmatory models. Psychological Assessment, 5, 216-229.

Leigh, B.C. \& Stacy, A.W. (2004). Alcohol expectancies and drinking in different age groups. Addiction, 99, 215-227.

Lemmers, L., Willems, Y., Thijssen, P., \& Osterman, C. (1998). Evaluatie zomercampagne 1997 'Lazer op met je zatte kop' [Evaluation summer campaign 1997 'Bugger off with your boozy talk']. Woerden: NIGZ.

Mackinnon, D. P. (1994). Analysis of mediating variables in prevention and intervention research. In: A. Cazares \& L. A. Beatty (Eds.). Scientific methods for prevention intervention research. pp. 127-153. NIDA Research Monograph No 139. NIH Publication 94-3631. Washington DC: Government printing office.

Mackinnon, D.P., Lockwood, C.M. \& Williams, J. (2004). Confidence limits for the indirect effects. Distrlbution of the product and resampling methods. Multivariate Behavioral Research, 39, 99-128.

Marlatt, G. A. \& Rohsenow, D. J. (1980), Cognitive processes in alcohol use: expectancy and the balanced placebo design. In N. K. Mello (Ed.), Advances in substance abuse: behavioral and biological research. pp. 159-199. Greenwich: JAI Press.

Mogg, K., Field, M. \& Bradley, B.P. (2005). Attentional and approach blases for smoking cues in smokers: An investigation of competing theoretical views of addiction. Psychopharmacology, 180, 333-341.

Musher-Eizenman, D. R. \& Kulick, A.D. (2003). An alcohol expectancy-challenge prevention program for at-risk college women. Psychology of Addictive Behaviors, 17, 163-166. 
Pos, S., Knibbe, R. \& Lemmers, L. (2001). Alle dagen feest? Een kwalitatieve studienaar omgevingsinvloeden op het alcoholgebruik van jongeren op vakantie [Party everyday? A qualitative study of environmental influences on young people's alcohol use on holiday]. Woerden/Maastricht: NIGZ/UM.

Schwartz, N. (1999). Self-reports. How questions shape the answers. American Psychologist, 54, 93-105.

Shrout, P. E. \& Bolger, N. (2002). Mediation in experimental and nonexperimental studies: new procedures and recommendations, Psychological Methods, 7, 422-445.

Stevens, J. (1992). Applled multivarlate statistics for the social sciences. (2 $2^{\text {nd }}$ edition). Hillsdale: Lawrence Erlbaum.

Van Dijck, D. \& Knibbe, R.A. (2005). De prevalentie van probleemdrinken in Nederland [The prevalence of problem drinking in The Netherlands]. Maastricht: UM

Van de Luitgaarden, J., Wiers, R.W., Knibbe, R.A. and Boon, B.J. (2006). From the laboratory to real life: A pilot study of an expectancy challenge with 'heavy drinking' young people on holiday. Substance Use and Misuse, $41,353-368$.

Wiers R.W. (2002). Half full or half empty, what are we drinking? Some comments on the discussion of the causal role of alcohol expectancies as a mechanism of change. Addiction, 97, 599-600.

Wiers, R.W. \& Hoogeveen, K.J. Sergeant, J.A. \& Gunning, B.W. (1997). High- and low-dose alcohol-related expectancies and the differential associations with drinking in male and female adolescents and young adults. Addiction, 92, 871-888.

Wiers, R.W., Van de Luitgaarden, J., Van den Wildenberg, E., \& Smulders, F.T.Y. (2005). Challenging implicit and explicit alcohol-related cognitions in young heavy drinkers. Addiction, 100, 806-819.

Wiers R.W., Van Woerden, N., Smulders, F. T. Y. \& De Jong, P. J. (2002). Implicit and explicit alcoholrelated cognitions in heavy and light drinkers. Journal of Abnormal Psychology, 111, 648-658. 


\title{
CHAPTER 6
}

\author{
How easy is it to get drunk? \\ Contextual factors related to \\ young people's excessive \\ alcohol use on holiday
}




\begin{abstract}
This study aimed to determine the influence of contextual factors (e.g. age checks, peer group) on young people's alcohol consumption on holiday. Young men ( $N=$ 492 ) aged 16 to 24 who were spending a vacation in seaside camping resorts in the Netherlands participated in the study. Several types of contextual factors; demographic factors; and alcohol consumption in daily life and on vacation were assessed with paper and pencil measures. Results show that several contextual factors are positively associated with alcohol use (i.e. number of male friends, switching ageidentifying wristbands, and money). However, none of the measures designed to limit consumption was significant. This suggests that formal control on youth drinking needs to be improved.
\end{abstract}




\subsection{Introduction}

Young men on holiday in seaside camping resorts in the Netherlands often drink excessive amounts of alcohol during their stay. Alcohol consumption generally starts soon after they wake up and continues throughout the day, resulting in an average consumption of 22 Dutch standard drinks (which contain 10 grams of pure alcohol) per day in men (Lemmers, Willems, Thijssen, \& Osterman, 1998). This heavy episodic binge drinking is related to a wide variety of health risks, including alcoholrelated traffic accidents, falls, fights, and alcohol poisoning (Van de Hoef, Lemmers, \& Knibbe, 2001). Additionally, excessive alcohol consumption is known to increase the likelihood of unsafe sex (Cooper, 2002; Fergusson \& Lynskey, 1996; Morgan, Plant, \& Plant, 1990).

Binge drinking by youth on holiday is not a typical Dutch phenomenon. Young people all over the western world use their vacation to drink to excess, for example students in North America often binge drink during spring break (Smeaton, Josiam, \& Dietrich, 1998), and in the Nordic countries many people drink excessively during the Midsummer festivities that coincide with the start of the summer holidays (Makela, Martikainen, \& Nihtila, 2005; Ramstedt, 2006). The context in which youth on holiday consume alcohol is in many ways different from the one at home. From the young people's perspective, the most appreciated characteristic of the holiday situation is that they are allowed to kick over the traces and are free from critical comments from parents and other authorities.

In the Netherlands, interventions aimed at increasing knowledge of the risks of heavy drinking have not been successful in reducing alcohol consumption in youth on holiday (Lemmers et al., 1998). The absence of direct impact of knowledge on young people's drinking when on holiday is not very surprising if one realizes that those young people who go on holiday without parents, are a selection of adolescents drinking considerably more than the average 16-25 year olds in the Netherlands (see first paragraph of results). One can assume that most young people on holiday want to drink alcohol. In that case, environmental interventions making it more difficult to drink may be more effective than interventions that aim to increase self control. This study is therefore explicitly focussed on contextual factors possibly explaining young people's alcohol consumption when on holiday. We have included three types of contextual factors that may influence alcohol use : (1)Variables indicating formalized and external control of possibilities to drink (e.g. checking ID card, using age-identifying wristbands), (2) variables indicating ways in which young people try to avoid formal control (e.g. switching wristbands, asking older persons to buy alcohol), and (3) variables indicating informal control by peers or adults (e.g. size and composition of the peer group, comments on alcohol use). Price of alcohol is of course also an important contextual variable influencing young people's alcohol consumption (Chaloupka, Grossman, \& Saffer, 2002). As price is rather uniform in all locations we included "money available to spend" as an indicator of variation between young adults in how easy it is for them to buy and consume drinks. 
In this article, the three main research questions are: Firstly, how many young people report to be exposed to particular contextual factors while on vacation. Secondly, which of the contextual factors are associated with young people's alcohol consumption when on holiday, and thirdly, what is the cumulative explanatory value of contextual factors in this study.

\subsection{Methods}

\subsubsection{Participants}

Young men ( $N=492$, mean age 17.9 years, minimum 16.0 years) who were spending a holiday on camping-sites in one of three seaside municipalities (Terschelling $n$ $=186$; Texel $n=115$; and Schouwen-Duiveland $n=191$ ) were invited to take part in the study by male and female trained students ('peers'). Young men were approached at their tents on the camping site. Non-cooperation was not systematically recorded, however the peers reported most men accepted the invitation, only very few declined the offer. To diminish interdependence between respondents, a maximum of two participants per drinking group (i.e. tent) were included. Participants were offered a financial incentive of $\sim 6$ Euros upon completion of the questionnaires. After agreeing to participate, subjects first filled out an informed consent form. Next, they completed questionnaires. Because only one measurement was used in the analyses, there was no dropout.

\subsubsection{Municipalities}

Terschelling and Texel are islands located in the North of The Netherlands. Terschelling has approximately 4740 inhabitants; Texel has a population of approximately 13750 people. Schouwen-Duiveland is a seaside municipality located in the southwest of the Netherlands, with a total population of around 34500 inhabitants. The summer population is much higher in all three places with an overrepresentation of youth.

\subsubsection{Materials}

Demographics Questionnaire. Participants were asked to indicate their age, housing situation (e.g. with parents, on one's own), romantic relationships, peer group size and composition during this holiday, length of stay, education, and amount of money that could be spent freely. Participants also indicated if they attended school, held a job, or both.

Drinking Questionnaire. Alcohol use was assessed with a general drinking questionnaire, including questions on alcohol consumption during ordinary weekdays, weekends, and evenings out (at home as well as in bars and disco's). Additionally, consumption on holiday was measured by a drinking diary of alcohol use during the previous day. Bars were open every day of the week, and the vast majority of young 
men on holiday go out every night, thus participants were recruited on all days of the week.

Contextual factors during the holiday. We included questions on the three types of contextual factors identified in the introduction: First, to measure formalized and external control of possibilities to drink, participants reported how often they had visited a pub or disco where they were asked for age-identification at the door. Participants also reported if they had been in a bar or disco where they were not asked for age identification at the door, and if the barkeeper asked for age identification at the bar when ordering in that case. Furthermore, participants answered questions on age checking when buying alcoholic drinks from supermarkets, camping stores, or liquor stores, and reported if a shop owner had refused to sell them any alcohol because they could not prove their age. They were also asked if they were aware of any rules regarding alcohol use and possession on the campsite, and if so, the contents of the rules they were aware of. Questions on how often they received or bought a wristband that identified them as being under 16 years of age, between 16 and 18 years of age, or over 18 years of age were also included. Wristbands were supposed to make it easier for sellers of alcohol to identify whether they were allowed to sell beverages with lower alcohol content (e.g. beer, wine) for which the minimum age is 16 years or also strong alcoholic beverages for which the minimum age is 18 years. These wristbands were provided to youth by personnel of several camping sites, bars and disco's (after age identification had been checked), and were also sold for $€ 1$ by a taxi company. Second, to measure the extent to which young people try to avoid formal control, participants were asked to report if they had switched wristbands with older people during the holiday, and if they had asked older persons to order alcohol for them. Third, to assess informal control by peers or adults, participants reported the size and composition of their peer group, and indicated if they had received comments on alcohol use by friends, partner, barkeeper, campsite manager, doorman, or parents. In addition to these three types of contextual factors, "money available to spend" was included as an indicator of variation between young men in how easy it is for them to buy and consume drinks.

\subsection{Results}

\subsubsection{Characteristics of young men on holiday}

The vast majority of participants $(91.9 \%)$ were on holiday with friends, without parents or partner. In daily life, nearly all lived with their parents (95.9\%). Their circle of friends included 7.4 persons on average (including themselves). Participants reported drinking an average of 24.2 Dutch standard drinks per day during vacation and an average of 35.6 standard drinks in a regular week when not on vacation. This is much more than the average weekly consumption for men in this age group in the Netherlands (15.5 standard drinks: Van Dijck \& Knibbe, 2005). Participants across all three municipalities were highly comparable on relevant factors. 


\subsubsection{Exposure to contextual factors}

To evaluate which contextual factors youth on holiday are exposed to, we examined the frequency distribution of contextual variables (see Table 1).

Variables indicating formalized and external control of possibilities to drink. As Table 1 shows, $66 \%$ of respondents reported that their ID card had been checked at least once when entering a bar or disco during the holiday. A lower percentage of youth reported their age was checked in an off premise store $(37.5 \%)$ or when ordering at a bar (9.5\%). Furthermore, approximately half of participants had used a wristband to demonstrate their age, and awareness of rules on alcohol use on the camping site was reported by 41.4 percent of young men. This indicates that formalized and external control on youth drinking could be subject to improvement.

Variables indicating ways in which youngsters try to avoid formal control. A relatively small proportion of young men try to avoid formal control by switching wristbands $(7.6 \%)$ and asking older persons to buy alcoholic beverages for them $(12.7 \%)$.

Variables indicating informal control by peers or adults. In terms of informal control the large number of friends (almost 6 on average) with whom one spends the holiday stands out. A minority of young men (28.7\%) had received comments on their drinking behaviour.

Money. The amount of money one can spend per month is about 300 euro although there is a large variation in that respect $(S D=270.04)$.

Table 1. Frequency distribution of contextual factors

\begin{tabular}{lll}
\hline Contextual factors & $M$ & $S D$
\end{tabular}

Variables indicating formalized and external control

Use of a wristband to demonstrate age $\quad 52.8$

ID card checked when entering a bar or disco $\quad 66.0$

ID card checked when ordering alcohol at the bar $\quad 9.5$

ID card checked in off premise store $\quad 37.8$

Alcohol purchase denied after failing to show valid ID 12.6

Being aware of rules on alcohol on the camping site $\quad 41.4$

Variables indicating avoidance of formal control

$\begin{array}{ll}\text { Having switched age- identifying wristband } & 7.6\end{array}$

$\begin{array}{lr}\text { Having asked older persons to buy alcohol } & 12.7\end{array}$

Variables indicating informal control

$\begin{array}{ll}\text { Presence of female friends in the peer group } & 22.8\end{array}$

$\begin{array}{lll}\text { Number of male friends in the peer group } & 5.94 & 3.47\end{array}$

Having received comments on drinking behavior during the holiday 28.7

$\begin{array}{lll}\text { Amount of money to spend per month } & 298.33 \quad 270.04\end{array}$

Note. Mean $(M)$ and standard deviation $(S D)$ were presented for continuous variables, for dichotomous variables the percentage of subjects who answered 'yes' was noted. 


\subsubsection{Contextual factors bivariately related to alcohol consumption of youth on holiday}

Bivariate analyses tested if significant differences in alcohol use on holiday were present between participants who reported being exposed to specific contextual factors, and those who reported not having been exposed. Table 2 shows results of these analyses. None of the factors indicating formal external control appear to be related to alcohol consumption. Of the factors indicating avoidance of formal control, switching age wristbands was positively associated with alcohol use. Three of the variables indicating informal control were significant in the bivariate analyses: Presence of female friends in the peer group; number of male friends in the peer group, and having received comments on drinking behaviour were all positively related to amount of alcohol consumed on holiday. The amount of money young men could spend was also significantly related to consumption.

\subsubsection{Cumulative explanatory value of contextual factors}

As predictor variables tend to be interrelated, bivariate analysis is unlikely to identify the most important contextual factors associated with alcohol use on holiday. To determine the relative contribution of contextual variables to the explanation of the variance in alcohol use on holiday, a stepwise forward regression was conducted (Table 3). Alcohol use on holiday was the dependent variable. Demographic variables were controlled for on the first step (age, being involved in a steady relationship, going to school, having a job). On the second step, contextual factors that were significantly associated with alcohol use in the bivariate analyses were added to the model. On the third step, alcohol use in daily life was entered. We chose to enter ordinary alcohol use last because the focus of this article is on exploring contextual factors, which may become statistically less significant after previous consumption (a known predictor) is added to the model. Table 3 shows the results of this analysis. Number of male friends in the peer group, switching age wristbands, and amount of money to spend were significantly associated with alcohol use on holiday after alcohol consumption in ordinary life was entered into the model. Every additional male friend in the group was associated with a 0.8 standard drink increase in alcohol consumption per vacation day $(p<0.001)$. The more money there was to spend, the more alcohol was consumed $(p<0.05)$; Young men who switched wristbands drank 7.45 standard drinks more, on average, than men who did not $(p<0.05)$. As expected, previous drinking was a significant predictor of alcohol use on holiday $(p<0.001)$. Contextual factors alone explained 16 percent of variance in vacation consumption.

\subsection{Discussion}

This study aimed to explore contextual factors associated with alcohol use in young men on holiday. Our results indicated that young men on holiday are exposed to a 
Table 2. Bivariate analysis of associations between contextual factors and alcohol use on holiday.

\begin{tabular}{|c|c|c|c|}
\hline Contextual factors & & $\begin{array}{l}\text { Mean alcohol use } \\
\text { on vacation day }\end{array}$ & $p$ \\
\hline \multicolumn{4}{|l|}{ Variables indicating formalized and external control } \\
\hline \multirow[t]{2}{*}{ Use of an armband to demonstrate age } & yes & 25.02 & \\
\hline & no & 23.35 & .18 \\
\hline \multirow[t]{2}{*}{ ID card checked when entering bar / disco } & yes & 23.66 & \\
\hline & no & 24.47 & .56 \\
\hline \multirow[t]{2}{*}{ ID card checked when ordering alcohol at the bar } & yes & 23.29 & \\
\hline & no & 24.90 & .54 \\
\hline \multirow[t]{2}{*}{ Awareness of rules on alcohol use on the camping site } & yes & 23.69 & \\
\hline & no & 24.56 & .48 \\
\hline \multirow[t]{2}{*}{ ID card checked in off premise store } & yes & 23.91 & \\
\hline & no & 24.46 & .66 \\
\hline \multirow[t]{2}{*}{ Alcohol purchase denied after failing to show valid ID } & yes & 26.35 & \\
\hline & no & 23.94 & .19 \\
\hline \multicolumn{4}{|l|}{ Variables indicating avoidance of formal control } \\
\hline \multirow[t]{2}{*}{ Having switched age-identifying wristband } & yes & 31.06 & \\
\hline & no & 23.70 & $<.01$ \\
\hline \multirow[t]{2}{*}{ Having asked older persons to buy alcohol } & yes & 25.80 & \\
\hline & no & 24.04 & .34 \\
\hline \multicolumn{4}{|l|}{ Variables indicating informal control } \\
\hline Number of male friends in the peer group & & Pearson's $r=.239$ & $<.001$ \\
\hline \multirow[t]{2}{*}{ Presence of female friends in the peer group } & yes & 27.53 & $<.01$ \\
\hline & no & 23.30 & \\
\hline \multirow[t]{2}{*}{ Received comments on drinking behavior during the holiday } & yes & 26.3823 .37 & $<.05$ \\
\hline & no & & \\
\hline Amount of money to spend freely each month & & Pearson's $r=.241$ & $<.001$ \\
\hline
\end{tabular}

Note. For number of male friends and amount of money to spend, correlations (Pearson's $r$ ) with alcohol use on holiday were reported.

variety of contextual factors, but that only a few of these factors are significantly related to alcohol use. Variables indicating formalized and external control of possibilities to drink were not related to alcohol use on holiday in our analyses. Of variables indicating avoidance of formal control, i.e. switching armbands and asking older persons to buy alcohol, only the first one was significant, in both bivariate and multivariate analyses. Several variables indicating informal control on youth drinking were significantly associated with alcohol use bivariately: Number of male friends, presence of female friends in the peer group, and having received comments on drinking behaviour were all positively associated with amount of alcohol consumed. However, in the stepwise regression, only number of male friends re- 
mained significant. Finally, money to spend was positively related to alcohol use on holiday.

Why were variables indicating external formal control not associated with alcohol consumption on holiday? In the Netherlands, compliance with alcohol laws (e.g. checking age of buyer, refusing alcohol to underage youth) is a problem, due to low enforcement by the Food and Consumer Product Safety Authority (FCPSA). The FCPSA has the jurisdiction to investigate violations of the law, however because alcohol sellers are inspected only once a year at most, law violations go largely unnoticed. As a result, external formal control is hardly present. It seems that young people in the Netherlands do not meet significant resistance when buying or drinking alcohol. This finding is in line with results of other studies, that found very high alcohol availability for Dutch youth (Bieleman, Kruize, \& Nienhuis, 2006), as well as a tolerant attitude towards youth alcohol use (Van den Eijnden, 2006; Van Hoof, 2005).

Table 3. Stepwise regression analysis to evaluate the explanatory value of contextual factors

\begin{tabular}{|c|c|c|c|c|c|c|}
\hline & \multicolumn{2}{|c|}{ Step 1} & \multicolumn{2}{|c|}{ Step 2} & \multicolumn{2}{|c|}{ Step 3} \\
\hline & $\beta$ & $p$ & $\beta$ & $p$ & $\beta$ & $p$ \\
\hline \multicolumn{7}{|l|}{ Demographic variables } \\
\hline Age & -0.53 & 0.23 & -0.77 & 0.08 & -0.33 & 0.40 \\
\hline Being involved in a steady relationship & 2.19 & 0.16 & 0.73 & 0.62 & 1.13 & 0.38 \\
\hline Going to school & -4.16 & 0.05 & -0.76 & 0.71 & -1.81 & 0.32 \\
\hline Having a job & 2.58 & 0.06 & 1.12 & 0.39 & -0.06 & 0.96 \\
\hline \multicolumn{7}{|l|}{ Contextual factors } \\
\hline Presence of female friends in the peer group & & & 2.27 & 0.11 & 0.35 & 0.78 \\
\hline Number of male friends in the peer group & & & 1.01 & $<0.001$ & 0.80 & $<0.001$ \\
\hline Switching age identifying wristbands & & & 7.45 & $<0.01$ & 4.45 & $<0.05$ \\
\hline $\begin{array}{l}\text { Having had comments on drinking behavior } \\
\text { during the holiday }\end{array}$ & & & 2.38 & 0.08 & 1.85 & 0.13 \\
\hline Amount of money to spend freely each month & & & 0.02 & $<0.001$ & 0.01 & $<0.05$ \\
\hline \multicolumn{7}{|l|}{ Previous alcohol consumption } \\
\hline \multirow{2}{*}{\multicolumn{2}{|c|}{ Total weekly alcohol consumption in ordinary life }} & & & & 0.27 & $<0.001$ \\
\hline & & $R^{2}=0.03$ & & $R^{2}=0,19$ & & $R^{2}=0.37$ \\
\hline
\end{tabular}

Table 1 shows that the proportion of youth trying to avoid formal control is relatively small. This is undoubtedly because alcohol laws are hardly enforced, and youngsters do not have to evade alcohol laws to obtain alcohol. Nevertheless, this result can also be taken to indicate that it does make sense to increase formal control, and that such an increase should include measures against the relatively small proportion of people trying to avoid it (e.g. by switching age-identifying wristbands). In addition, increasing the proportion of young people who have to wear wristbands to be able to buy alcohol at bars or off premise stores may help to reduce drinking 
among a large part of the adolescents who as yet do not have to wear a wristband to buy alcohol.

Several variables indicating informal control were significant in our analyses. Almost $30 \%$ of young adults got one or more critical comments on their drinking during the holiday, by friends, partner, barkeeper, campsite manager, bouncer, or parents. The bivariate and multivariate analyses show that these comments are aimed at those who drink even more than the others. Number of male friends in the peer group was significantly associated with alcohol use. On holiday, alcohol consumption mainly takes place in large drinking groups. Research by Van de Goor, Knibbe and Drop (1990) indicates that young men drink faster when they participate in groups of four or more members. The larger the (all-male) drinking group, the more alcohol is consumed. Our results are in line with this. Previous research found that drinking rates differ significantly between girls and boys, and that the presence of females in the drinking group led to a somewhat slower drinking rate (as compared to an all-male group, Van de Goor et al., 1990). In contrast, in this study presence of at least one female friend was bivariately associated with a significantly higher alcohol consumption. The presence of females in the peer group did not limit their male friends' alcohol use.

Amount of money available to spend was a significant predictor of alcohol use on holiday. The more money one can spend the more alcohol one is able to buy. Because the large majority of young men on holiday go to school in regular life, they have limited resources for buying alcohol on holiday. Increasing alcohol prices may therefore help to reduce consumption.

The present study is restricted by the fact that only a limited number of contextual factors were measured and analyzed. There are other types of contextual factors that may be associated with alcohol consumption on holiday, which we have not included in our analyses (e.g. opening hours and density of bars).

In conclusion, the most surprising outcome of our study is not that variables indicating informal control are associated with alcohol use. Rather, it is the lack of significance of variables indicating formal control on youth drinking that is the most relevant outcome from a policy point of view. The fact that variables indicating formal control do not explain any variation in alcohol use on holiday, suggests that the most promising way to make it more difficult for young people to become drunk is to substantially increase formal control on young people's drinking. 


\section{References}

Bieleman, B., Kruize, A., \& Nienhuis, A. (2006). Naleving leeftijdsgrenzen 16 en 18 jaar Drank-en Horecawet. Metingen 1999, 2001, 2003 en 2005 [Compliance with minimum ages, 16 and 18, as stated in the Alcohol licensing and catering act. Measurements 1999, 2001, 2003 and 2005]. Groningen: Stichting Intraval.

Chaloupka, F. J., Grossman, M., \& Saffer, H. (2002). The effects of price on alcohol consumption and alcohol-related problems. Alcohol research and health, 26, 22-34.

Cooper, M. L. (2002). Alcohol use and risky sexual behaviour among college students and youth: evaluating the evidence. Journal of Studies on Alcohol, 14, 101-117.

Fergusson, D. M., \& Lynskey, M. T. (1996). Alcohol misuse and adolescent sexual behaviors and risk taking. Pediatrics, 98, 91-96.

Lemmers, L., Willems, Y., Thijssen, P., \& Osterman, C. (1998). Evaluatie zomercampagne 1997 'Lazer op met je zatte kop' [Evaluation summer campaign 1997 'Bugger off with your boozy talk']. Woerden: NIGZ.

Makela, P., Martikainen, P., \& Nihtila, E. (2005). Temporal variation in deaths related to alcohol intoxication and drinking. International journal of Epidemiology, 34(4), 765-771.

Morgan, R., Plant, M. A., \& Plant, M. L. (1990). Alcohol, AlDS risks and sex industry clients: results from a Scottish study. Drug and alcohol dependence, 26, 265-269.

Ramstedt, M. (2006). Alcohol poisonings in Sweden 1987-2004. Trends and temporal patterns in times of a rapidly rising population drinking, Nordic studies on Alcohol and Drugs, 23, 97-109.

Smeaton, G. L., Josiam, B. M., \& Dietrich, U. C. (1998). College students' binge drinking at a beach-front destination during spring break. Journal of American College Health, 46, 247-254.

Van de Goor, L. A., Knibbe, R. A., \& Drop, M. J. (1990). Adolescent drinking behavior: an observational study of the influence of situational factors on adolescent drinking rates. Jourmal of Studies on Alcohol, 51, 548-555.

Van de Hoef, P., Lemmers, L., \& Knibbe, R. A. (2001). Jongeren, alcohol en vakantie: Kwantitatieve studie naar het alcoholgebruik van jongeren op vakantie in Nederland en Spanje IYoung people, alcohol, and holiday: A quantitative study on the alcohol use of youngsters on holiday in the Netherlands and Spain]. Woerden: NIGZ.

Van den Eijnden, R. J. J. M. (2006). Alcoholgebruik jongeren 12-17 jaar: De rol van de ouders [A/cohol use among young people between the ages of 12-17: The role of parents]. Paper presented at the National Alcohol conference.

Van Dijck, D., \& Knibbe, R. A. (2005). De prevalentie van probleemdrinken in Nederiand [The prevalence of problem drinking in The Netherlands]. Maastricht: UM.

Van Hoof, J. (2005). Mixdrankjes uit de supermarkt? Onderzoek naar het koopgedrag van ouders van kinderen van 10 tot en met 15 jaar in relatie tot alcohol en mixdrankjes in het bijzonder [Mixed drinks banned from supermarket shelves? A study of parents of chlldren aged 10-15 and their buying behavior of mixed drinks and other alcoholic drinks]. Twente/Utrecht: University of Twente / STAP. 


\title{
CHAPTER 7
}

\author{
Adolescents binge \\ drinking when on holiday: \\ An evaluation of a community \\ intervention based on \\ self regulation
}




\section{Abstract}

This article presents a case study of a community intervention aimed at reducing excessive drinking in young men on holiday in seaside camping resorts in the Netherlands. The self-regulated voluntary covenant of parties concerned was evaluated on the basis of several types of data (all collected in 2004): questionnaires administered to young men ( $N=191)$, observations carried out by trained 'peers', nuisance questionnaires among city centre residents $(N=121)$, and interviews with local actors. Compliance with measures, as well as effects of measures are discussed. Limitations are noted, and recommendations for optimizing the potential of community interventions are made. 


\subsection{Introduction}

Alcohol is the most widely used drug among youth in the western world (Johnston, O'Malley, \& Bachman, 2000). An important factor is that in the Netherlands as well as in many other countries, adolescents perceive unsupervised alcohol consumption as a sign of transition from childhood into young adulthood (Engels, Knibbe, \& Drop, 1999). More specific for the Netherlands is that the attitude towards alcohol use by adolescents is very tolerant in general, and alcohol is widely available to youths (Van de Luitgaarden, Thush, Wiers, \& Knibbe, 2008). During the summer holidays, when spending a vacation on a campsite with peers, young people's alcohol use increases. Young men drink an average of 22 Dutch standard drinks per day and young women drink an average of 9 (Lemmers, Willems, Thijssen, \& Osterman, 1998). This period of daily binge drinking poses many risks, such as alcohol poisoning, alcohol-induced fights and traffic accidents, unprotected sex, and disturbances of public order (Van de Hoef, Lemmers, \& Knibbe, 2001).

Adolescence is a time in which young people are expected to experiment with risk-taking behaviors such as alcohol and drug use, sex, and smoking. Earlier studies on adolescents on holiday quite convincingly indicate that alcohol consumption in itself is the main risk factor both for direct consequences like alcohol induced coma's, accidents and for more indirect consequences like alcohol related violence and unwanted sex. Most likely, in the holiday situation, most of the health risks of young people are directly or indirectly related to drinking. In very many cases, all activities from waking up to falling asleep are accompanied by drinking alcohol.

To achieve successful intervention programs, it is important to identify predictors of use, in order to target them (Perry, 1999). Social, environmental and intrapersonal factors have consistently been found to be associated with adolescent alcohol use (Komro, Hu, \& Flay, 1997, Kosterman, Hawkins, Gui, Catalano, \& Abbott, 2000). On vacation, many of these factors are more stimulating of heavy drinking than in regular life. For example, absent parental supervision, lack of rules on alcohol use, peer alcohol use, and perceived peer approval of drinking stimulate youngsters to drink more than they otherwise would (Pos, Knibbe, \& Lemmers, 2001). Add this to the legal, economic, and physical availability of alcohol in the Netherlands and it is easy to conceive why so many young people are able to engage in risky drinking in this situation. The interaction between social, environmental, and personal characteristics calls for interventions that tap into the social world of adolescents on holiday: their peer group, the community environment and alcohol availability.

However, to move from evidence-based targets to intervention planning, implementation and effective sustaining of a program a number of conditions need to be met. Because in our study alcohol consumption during holiday was a central theme, an intervention aimed at the environment of the adolescents seemed to be more promising than an intervention aimed at the individual adolescents themselves. The support of the main actors within the community for such an intervention seemed promising because the local population had been annoyed by the nuisance caused by intoxicated young people on holiday for a longer time. Also the local 
council was very supportive of such an initiative in the hope that an adequate community intervention might prevent alcohol related health and nuisance in future summers as well.

Communities provide an attractive site for preventive interventions in the Netherlands, because they are able to implement local alcohol policies that are stricter than national alcohol laws (Ministry of Health, Welfare, and Sport, 2007; Ministry of the Interior and Kingdom Relations, 2007). Studies show that community interventions aimed at preventing youth access to alcohol can make an important contribution in the battle against youth alcohol abuse (Wagenaar, Toomey, \& Erickson, 2005; Stafström, Östergren, Larsson, Lindgren, \& Lundborg, 2006). Internationally, projects have included increasing age checks in bars through stricter enforcement of underage alcohol sales laws (e.g. Holder, Saltz, Grube, Treno, Reynolds, Voas, \& Gruenewald, 1997), training management of bars (Toomey et al., 2001), and changing cultural norms that tolerate underage drinking (e.g. Perry et al., 2002; Wagenaar, Murray, Wolfson, Forster, \& Finnegan, 1994).

Many community interventions on alcohol have been done in other countries (e.g. Perry et al., 2002; Stafström, Östergren, Larsson, Lindgren, \& Lundborg, 2006; Wagenaar, Toomey, \& Erickson, 2005), however this was the first time a community intervention on alcohol was attempted in the Netherlands. It is markedly different from those achieved elsewhere in two ways: First, because it is aimed at temporary residents, namely youth on holiday in the community. The community therefore only carries limited responsibility for the welfare of this population. Because of this, reducing public nuisance may be the most important motive for local actors to take part in the intervention. The second distinguishing factor is that this is a voluntary community intervention with little or no legal means to ensure compliance with it. This stands in contrast with other community interventions that typically rely, in part, on a fair amount of enforcement (e.g. Stafström et al., 2006). In the Netherlands, however, it is common to try self-regulation first, before resorting to official measures (Van de Luitgaarden, Thush, Wiers, \& Knibbe, 2008).

In the community intervention the measures included in the covenant were selected in two steps. In the first step relevant actors within the community le.g. shop owners, managers of pubs, restaurants, campings; police; general practitioners) were asked which measures they considered as promising to prevent excessive alcohol consumption. In a second step the measures to be included in the community intervention were decided upon on the basis of consent by all those who were involved in the community intervention working group. This group encompassed most of the relevant community stakeholders. The consensus about measures to be taken was formally established in a so called covenant.

The measures taken in this covenant can be classified according to two perspectives. First, a distinction can be made between measures that rely on cooperation of alcohol sellers, and measures that are dependent on implementation by other stakeholders. Second, national alcohol laws can be distinguished from new local regulations. This raises the question to which extent compliance with national alcohol rules improves due to the covenant, and to what degree the newly agreed upon local measures are complied with. 
This paper evaluates this community intervention in terms of a) compliance with measures agreed upon, b) effects on youth drinking, and c) effects local actors attributed to the intervention. The role the self-regulatory nature of the covenant played in the results will be discussed.

\subsection{Method}

\subsubsection{Study community}

The community project was conducted in the spring and summer of 2004 on Schouwen-Duiveland in the province of Zeeland, in the Netherlands. This island has become one of the most important tourist places on the Dutch coast in the past decennia. The masses know Renesse and to a lesser extent Westenschouwen, Burgh-Haamstede and Scharendijke for its beautiful nature and its lavish nightlife. With only 34,000 permanent inhabitants, the island is invaded by a large number of visitors during the summer season. The small community is to a large extent economically dependent on tourism. However, the increase of tourists has also caused problems, especially increases in thefts, fights, vandalism, noise pollution, and urinating in public. The image of Renesse became negative to the extent that it started to affect the number of tourists. In 1998, the municipality council decided that a local health policy was to be established in which alcohol use was named as a significant health risk (Municipality Schouwen-Duiveland, 2001). Policy documents show increased investments in law enforcement, an active prevention policy, and the aim to collaborate with all partners involved. Police and municipality are not the only ones responsible for local health and safety policies, as civil society, like the catering industry, shop owners, recreation entrepreneurs and individual citizens can contribute as well (Municipality Schouwen-Duiveland, 2004). According to some stakeholders, there was some decrease in public nuisance, but the problems were still not completely solved. The community wanted to emit a positive image, without associations with public disturbance by drunken youth. Measures as tit-for-tat policy, public transportation of youth from downtown bars to the camping sites, local bans on possessing and consuming alcoholic drinks in the public space and an alcohol education campaign in the summer have been used in the community for years (Municipality Schouwen-Duiveland, 2004). In addition, the municipality council of Schouwen-Duiveland decided to take part in the community intervention project of Maastricht University and the Netherlands Institute for Health Promotion and Disease Prevention (NIGZ) in 2004 when they were approached to participate.

\subsubsection{Community intervention}

Local alcohol policy group. The intervention was aimed at reducing alcohol use by youth on holiday through collective measures agreed upon in a covenant. A precondition for the establishment of such a covenant would be that relevant community actors which traditionally do not see it as their core task to take responsibility for limiting alcohol use, are made aware of their influence on drinking behaviour (cf. 
Treno, Gruenewald, Lee \& Remer, 2007) of young people. The community prevention project should be the joint responsibility of all in the partnership, both community and research participants (Holder et al., 1997).

To achieve this prerequisite, and in order to rally support for an intervention, a small working group was formed in January 2004 , consisting of a municipality representative, an employee of the local outpatients' services for alcohol and drug abuse, and the project manager of the NIGZ. Together, they made a list of organizations/actors that would be invited to participate in a newly formed alcohol policy group.

The selection of actors was based on an analysis of the various categories of stakeholders that could be considered to have a role in dealing with excessive alcohol use and its consequences. Actors or organizations were included on the basis of either providing alcohol to young people (supermarkets, liquor store, bars), responsibility for public order (police, local council, managers of campingsites were young people stayed) or dealing with health problems caused by heavy drinking youth (general practitioners, municipal health services). Subsequently, representatives of those groups were approached to take part in the policy group on a voluntary basis. Parents or youngsters did not form part of this group, as the group is municipalitybased and the intervention is aimed at youngsters from outside the community.

The mayor would chair meetings, and practical assistance would be given by officials. An employee of the NIGZ was the project manager, who collaborated closely with the prevention department of the local outpatients' services for alcohol and drug abuse. Only a subset of all relevant organizations had attended the first meeting. To ascertain that representatives of all relevant local organizations (bar owners, managers of supermarkets and liquor stores, camping attendants, health workers, general practitioners, and police officers) supported the new initiative, the project manager interviewed everyone involved. After these interviews, nearly all local actors joined the alcohol policy group.

Measures agreed upon in the covenant. The project manager suggested measures for the covenant, based on the contents of the interviews with local actors, and the discussions in the local policy group. On June 14, 2004, a covenant was signed by 16 stakeholders involved with youth on holiday on the island. Table 1 details the measures that were agreed upon in the covenant. However, it is important to note that the covenant did not contain all recommendations that were made by the project manager. In particular: 1) an alcohol sales registration system was rejected by alcohol sellers as being too difficult to achieve in practice, 2) the suggestion to ban "walls" of beer cases on the camping sites was not included in the final version, 3) the proposal to prohibit youth under 16 years of age from visiting camping sites unattended by parents was weakened by changing the text to "age limits will be set for youth camping without parental supervision". One measure was added, namely the aim to use a system of age-bracelets in all bars and on all camping sites. Measures that could have legal consequences were resisted by parties concerned, therefore the final covenant was a gentlemen's agreement, with little enforceable measures (see Table 1). In addition to these specific contents of the covenant, all participants also explicitly agreed to adhere to the national Alcohol Licensing and Catering 
Act. This act states that sellers are required to verify the legal age of young buyers, and are only allowed to sell alcohol when the customer is at least 16 years old (18 for spirits). Sellers are obliged to make sure that young people over 16 or 18 do not buy alcoholic beverages for those younger than 16 or 18 . Additionally, the Dutch law states that bartenders have to refuse selling alcohol to someone who is intoxicated.

Table 1. Measures agreed upon in the covenant

Covenant on youth alcohol use Schouwen-Duiveland

1. If there is any doubt about the buyer's age, age identification will be asked.

2. All bars and cafés will use a system of bracelets that indicate the age of the visitor. This will make it easier to recognize underage youth. The aim is to develop a coordinated system of bracelets that is used by camping sites as well.

3. Adolescents who receive alcohol through older persons, whereas they are not allowed to buy it by law, are reprimanded by staff.

4. Camping attendants will try to limit excessive drinking on their property. All camping sites will have camping regulations that include rules on alcohol consumption. Age limits will be set for youth staying at camping sites without their parents.

5. It is prohibited to consume alcohol in public spaces. Police are authorized to seize alcohol and report offenders.

6. The tit-for-tat policy will be continued, with extra emphasis on preventing and intervening with drug dealing. A complaints office will be created where nuisance can be reported.

Enforcement of measures. No specific provisions were made in the covenant to ensure compliance with the regulations therein. In the Netherlands, the Food and Consumer Product Safety Authority (FCPSA) is the organization charged with enforcement of the Alcohol Licensing and Catering Act. Note that enforcement of alcohol laws is not the responsibility of the community, nor of the police. Several measures agreed upon in the covenant were already set out by national alcohol laws, and were therefore, in theory, enforced by the FCPSA. In practice, alcohol laws are hardly enforced in the Netherlands, as a result of structural understaffing of the FCPSA. The large majority of underage Dutch youth is able to buy alcohol in supermarkets and liquor stores (Gosselt, 2006). Proof of age is rarely asked, and alcohol sellers are inspected only once a year, at most. The covenant was essentially an agreement for which compliance depended on goodwill of local actors.

\subsubsection{Subjects}

The local policy group was initiated with the aim to reduce alcohol use in young men (ages 16-24) on holiday. To evaluate the covenant, trained 'peers' administered questionnaires to young men $(N=191)$ spending a holiday on the island during the summer. Participants were approached at the campsite, and were asked if they would like to take part in a study on youth alcohol use that would include filling out questionnaires. Participants received $€ 5$ for their participation. Table 2 reports background characteristics of participants. 
Table 2. Background characteristics of participants

\begin{tabular}{lccc}
\hline Background characteristics & $M$ & $S D$ & \% yes \\
\hline Amount of money to spend per month, in Euros & 325.19 & 293.03 & 4.47 \\
Size of circle of friends on holiday (excl. themselves) & 7.15 & 18.44 \\
Total alcohol consumption in a regular weekend in Dutch & 31.82 & \\
standard drinks & & 8.89 \\
Total alcohol consumption Monday -Thursday in Dutch & 8.02 & 1.66 \\
standard drinks & 18.05 & 96.3 \\
Age, in years & & 85 \\
Living with parents in regular life & & 79 \\
On holiday with friends only & & \\
Going to school in regular life & & \\
Holding a job (part-time or full-time) & & \\
In a relationship & & \\
\hline
\end{tabular}

\subsubsection{Data sources}

To evaluate the covenant, several data sources were used. A multi-method case study (Yin, 2003) was used to evaluate compliance with specific measures in the covenant, and effects ascribed to it. Data source triangulation was used to obtain a more accurate evaluation (Denzin, 1978). Data source triangulation entails the use of multiple data sources to evaluate a research question. For example, in the present study, compliance with age laws was evaluated by analyzing youth questionnaires, but also by interviewing alcohol sellers, and checking with the authorities if alcohol sellers had been fined for underage alcohol sales.

Each data source is described below. Youth questionnaires were administered in July and August of 2004, and observations on camping sites were done in this time period as well. In September 2004, nuisance questionnaires were administered and local actors were interviewed (by phone).

Youth Questionnaires. This questionnaire was used to measure 1) youth alcohol use, and 2) effects of specific regulations agreed upon in the covenant. To measure youth alcohol use, questions on alcohol consumption during ordinary weekdays, weekends, and evenings out were included. Additionally, consumption on holiday was measured by a drinking diary of alcohol use during the previous day (see Van de Luitgaarden, Wiers, Knibbe, \& Candel, 2007). Effects of specific measures agreed upon in the covenant were measured by questions on how often they had visited a pub or disco where they were asked for age-identification at the door, and how often they received or bought a bracelet (that identified them as being under 16 years of age, between 16 and 18 years of age, or over 18 years of age). In addition, they were asked if they had exchanged these bracelets with older people, if they had asked older persons to order alcohol for them, and if they themselves had bought alcohol for younger persons. It was also asked if they had been in a pub or disco where they were not asked for age identification at the door, and if the barkeeper asked for age identification at the bar when ordering in that case. Partici- 
pants also reported if a barkeeper had refused to serve them alcoholic drinks, and if they had received comments on their alcohol use from other persons (e.g. friends, partner, barkeeper, campsite manager, bouncer, parents). Furthermore, they were asked if they were aware of any rules regarding alcohol use and possession on the campsite, and if so, the contents of the rules they were aware of.

Interviews with local actors. In September 2004, all local actors who had collaborated on the covenant were interviewed by telephone by a university researcher. These interviews lasted 15-25 minutes, and centered on how the local actors themselves evaluated the covenant, in terms of implementation, and effects of measures. They were also asked if the covenant had any shortcomings, and if any incidents happened during the summer.

Observations on camping sites. Trained 'peers' visited camping sites and observed its characteristics. Presence of a doorman, age distribution of visitors, percentage of men vs. women, distribution of the number of cases of beer at the tents, and the presence of "walls" of cases of beer was noted (a "wall" consists of at least 9 cases of beer). Finally, the number of apparently intoxicated young people (age < 25 ) at the camping site was estimated (note that youth sleeping off a hangover in the tent could not be counted).

Nuisance questionnaires. Empirically-based Nuisance questionnaires were filled out by residents from households $(N=121)$ in the city centre of Renesse. These households were handpicked by trained 'peers' because of their vicinity to bars and disco's. The questionnaire contained a total of 68 questions (open-ended, multiplechoice, and Likert type), and took 10 to 15 minutes to complete on average.

Questions addressed the amount of nuisance participants experienced from fighting and yelling people, drunk people, petty crime, threats / intimidations, physical violence, use of alcoholic drinks in the streets, use of cannabis or other drugs on the streets, drug-dealing in the neighbourhood, noise pollution from clubs and bars, littering by bar visitors, accidents involving drunk people, auto crime, bike thefts and vandalism, robberies, graffiti on buildings and walls, and people urinating on the streets.

Data on alcohol use in previous years. To evaluate if the covenant had any effect on youth drinking, data from this study are compared to data collected two years earlier in the same community.

\subsection{Results}

Results of the community intervention can be divided into three categories: 1) compliance with measures, 2) effects on youth drinking during the holiday, and 3) effects the local actors attributed to the covenant. 


\subsubsection{Compliance with measures}

Compliance with measures agreed upon in the covenant (see Table 1) was studied by analyzing youth questionnaires, interviews with members of the alcohol policy group, observations on camping sites, and results from nuisance questionnaires.

In doubt, ask identification. Thirty-eight percent of respondents (all 16+) indicated that they were asked to show age-identification before entering a bar or disco at least once during the vacation. An additional $6.1 \%$ of $16-17$ year old young men were asked for identification when ordering alcoholic drinks at the bar lat least once). None of the subjects were asked for identification when buying alcoholic drinks at a supermarket or liquor store. Note that in the Netherlands, adolescents are allowed to buy beer and wine from the age of 16 , and spirits from the age of 18 . It is unknown how many adolescents younger than 16 years of age were asked for identification when buying alcohol from bars and cafés. During the qualitative interviews local actors reported that several bar owners were fined for serving alcohol to underage patrons during the summer. They indicated that compliance was difficult in practice, because young people would pass alcohol to younger friends, and the bar environment (dark \& noisy) made it difficult to ask for identification.

System of bracelets that indicate the age of the visitor. Data from the questionnaires indicate that $53.4 \%$ of young men on holiday on the island wore a bracelet that indicated their age. $8 \%$ of 16- and 17 -year-olds had switched bracelets with other young people during their stay. Given that only $9 \%$ of participants had not bought any alcohol in a bar or disco during the holiday, it is obvious that a considerable percentage of bar and disco visitors did not wear the aforementioned bracelets. Peer observations at one of three camping stores indicated that the shop owner would only sell alcohol if the customer showed the bracelet. If the customer could not show the bracelet, an id-card or passport was required to verify the buyer's age. Qualitative interviews with local actors confirmed that the system for distributing bracelets during the summer had not worked out as planned. The idea was that youth would buy these bracelets for $€ 1$ at a taxi company in the city centre of Renesse. However, many young people did not buy the bracelet. Local actors indicated that they had already devised a plan to distribute the bracelets through the camping sites for free the next summer.

Underage adolescents, who receive alcohol through older persons, are reprimanded by staff. Because all participants in our study were over 16 years of age (the legal drinking age for low alcohol content beverages, e.g. beer and wine) it is not possible to fully report on the effect of this measure for younger youth. However, we were able to evaluate this measure in 16- and 17 -year olds, who are still underage for buying spirits. The youth questionnaires show that $13.6 \%$ of 16 - and 17 year-olds asked older friends to order spirits for them. However, only $3.3 \%$ of 16 and 17 year olds in total were reprimanded for their alcohol use $(1.1 \%$ by barkeeper, $2.2 \%$ by a doorman). This means that, at the very most, a quarter of adolescents who receive strong liquor through friends are caught and reprimanded. In addition, $34.6 \%$ of all respondents indicated having bought alcohol for underage friends during the holiday. Eight percent of respondents even switched agebracelets with older friends to be able to buy alcohol and get into bars. Local actors 
indicated that it was difficult for staff to know who consumed the beverages that they sold, as young people often buy drinks in rounds, and barkeepers do not see who the drinks are passed to. In supermarkets beer is usually bought by the case, and distributed to friends at the camping site or beach.

Camping attendants will try to limit excessive drinking on their property. The covenant stated two measures that could contribute to achieving this goal: 1) instating camping regulations that include rules on alcohol consumption, and 2) setting age limits for youth staying at camping sites without their parents. The youth questionnaires show that $35 \%$ of young men report awareness of camping rules regarding alcohol possession and use. It must be noted, however, that camping rules may have been present, without youth being aware of their existence. Eight percent of youth staying at camping sites reported being called to account for their alcohol use by the camping attendant. Peer observations on camping sites confirmed the supervising function of camping attendants. Peers did not notice any adolescents under 16 years of age staying at the camping site unattended by parents. Furthermore, peers observed that one of the most popular camping sites checked for litter several times a day. Cases of beer were not allowed outside of the tents. Adolescents on the spot indicated that if there was litter (incl. cases of beer) at the tent, the camping attendant woke them up at $8.30 \mathrm{a} . \mathrm{m}$. and they were instructed to clean the place up immediately.

No alcohol and drug use in the public space. Sixty-three percent of young men indicated having drunk alcohol on the beach during the holiday, despite the rule in the covenant banning consumption of alcohol in the public space, which aimed to prevent this. Nuisance questionnaires showed that $39.5 \%$ of city centre residents were bothered by drunken people on the streets. Furthermore, $57.1 \%$ was annoyed by people urinating in public and $69.8 \%$ was bothered by fights and yelling on the streets (which are typically drunk behaviors). Nuisance questionnaires showed that the vast majority (80-90\%) of city centre residents did not notice any trouble caused by drug use or drug dealing on the streets.

Tit-for-tat policy will be continued. The police indicated that approximately the same number of offences was reported as in previous years. Most offences involved alcohol consumption in the public space and urinating in public.

\subsubsection{Effects on youth drinking}

When it comes to effects on alcohol consumption, it must be noted that it is not possible to draw firm conclusions on the basis of the available data. However, it is possible to get an indication by comparing alcohol use during the summer of 2004 to consumption in the same community two years earlier (Van de Luitgaarden et al., 2006). In 2002, young men drank an average of 23.7 standard drinks per vacation day $(S D=15.38)$, while they drank 25.3 glasses on average in 2004 . This was in the same range as comparable communities, possibly even somewhat higher (Van de Luitgaarden, Wiers, Knibbe, \& Candel, 2007). It is evident that the problem of youth drinking to excess was not reduced. Thus, alcohol use among young people on holi- 
day on Schouwen-Duiveland was excessive before the community intervention, and was still excessive after the intervention.

\subsubsection{Effects attributed to the covenant by members of the policy group}

Based on their own experiences, local actors ascribed several effects to the covenant: an increased awareness of the problem among parties concerned, easier identification of underage youth (even though distribution of bracelets was not optimal), and less public nuisance (mainly because of the tit-for-tat policy). However, most respondents to the nuisance questionnaire $(53 \%)$ indicated that nuisance was of the same magnitude as the previous year, while $7 \%$ reported an increase in public nuisance, and $37 \%$ reported a decrease. When asked what they thought was the reason nuisance had decreased, the only explanation given was the bad weather during the summer.

Many local actors indicated that changes take time, and that the alcohol policy group had only been instated recently. Several local actors suggested improvements to the current measures and a few new measures to come into effect the next summer (e.g. another distribution system for age-bracelets, more effective communication of camping rules, and so called "intervention teams" to prevent public nuisance).

\subsection{Discussion}

Local actors in Schouwen-Duiveland were willing to work together on the problem of excessive drinking by youth spending a holiday in the community. A covenant on alcohol was signed; however measures agreed upon were fairly limited. A relatively large portion of the "new" regulations (e.g. rules on age limits) were already set out by the national "Alcohol Catering and Licensing act"; they were just never strictly enforced. Other regulations focused on reducing public nuisance by fining citizens through the tit-for-tat policy. Alcohol sellers did not meet new regulations, apart from the age bracelets, which were not distributed as widely as planned (only a relatively small percentage of youth visiting the island wore an age bracelet). Camping attendants were the only ones that took on new regulations. The main commercial interests were kept out of range, and the formulation of the regulations kept legal consequences in case of non-compliance away. Some attention was given to the new local regulations, however compliance with national alcohol laws was still poor. Overall, it can be noted that measures that relied upon co-operation of alcohol sellers were more poorly complied with than measures carried out by other stakeholders. In the covenant, no provisions were made to ensure compliance of stakeholders with any of the measures. Therefore, it is not surprising that the lack of compliance with alcohol laws continued to be a key problem, as evidenced by the small percentage of youth who had been asked for identification. During the summer, several alcohol sellers were fined for selling alcohol to underage youth. Their reaction to this is tell-all: it was considered harsh that they were fined, since they 
had collaborated on development of the covenant so willingly. This is illustrative for the fact that the covenant was a "gentlemen's agreement" instead of a deal they could be held accountable for. Apparently, their commitment to the covenant was not enough to ensure compliance with it. During the development of the covenant, little thought was given to means of enforcing it. As a result, the proposed selfregulation only entailed limited loyalty to small goals. By allowing alcohol sellers to seif-regulate, they are given the possibility to avoid regulations that are likely to be the most effective.

A related problem is the emphasis several local stakeholders placed on preventing public nuisance instead of reducing actual drinking. It is questionable if they really shared the public health aim of limiting alcohol use, rather than just reducing public nuisance. Measures that reduce public nuisance (e.g. tit-for-tat policy, prohibiting alcohol use in the public space) were supported by all members of the policy group, whereas measures that could potentially reduce drinking by reducing alcohol sales (e.g. registration system of alcohol sales to limit alcohol sold to young people) were dismissed before composition of the covenant. It is important to remember that the study community is to a large extent economically dependent on tourism. Therefore, certain measures that were considered to be a threat to community members' income were quickly rejected. This weakened the overall content of the covenant.

A further problem was that the time frame in which the alcohol policy group had to be started, support of local actors had to be gained, and the covenant had to be composed and signed was way too short. A community intervention is often a relatively slow process which does not produce large results in just a few months time. In order to achieve more meaningful results more preparation time is needed.

In the Netherlands, community interventions on alcohol are scarcely out of the egg. The community intervention project on Schouwen-Duiveland was one of the first projects that were undertaken, and the problems that were encountered can teach prevention workers four valuable lessons for future studies. First, the necessity of all participants being on the same page when it comes to defining the problem. Second, the importance of allowing enough time for the whole process, from getting local actors together to formulating and executing the measures taken. Third, the importance of agreeing on measures that can be legally enforced, and fourth, the importance of law enforcement. In contrast with the Dutch tradition to rely on industry self-regulation, it is important to recognize that purely voluntary covenants do not work in the field of alcohol abuse, because separate, conflicting and irreconcilable interests are present. Therefore, a certain degree of legal pressure is necessary to ensure that local actors keep their commitment to the covenant. At the moment, the local community does not have the power to arrange for law enforcement, and the FCPSA does not have the manpower to enforce the Alcohol Licensing and Catering Act effectively. This is an important obstacle, which needs to be solved to optimize the potential of community interventions on alcohol in the Netherlands. 


\section{References}

Denzin, N.K. (1978). The research act: A theoretical introduction to sociological methods (2nd ed.). New York: McGraw-Hill,

Engels, R.C.M.E., Knibbe, R.A., \& Drop, M.J. (1999) Visiting public drinking places: an explorative study into the functions of pub going for late adolescents. Substance $U$ se and Misuse, 34,1061-1080.

Gosselt, J.F. (2006). Drank kopen kent geen leeftijd. Alcoholverkoop aan jongeren onder de wettelijk toegestane leeftijdsgrens: een onderzoeksprotocol en een studie naar de naleving van de Nederlandse Drank- en Horecawet [Buying alcohol is ageless. Alcohol sales to underage youth: a research protocol and a study of compliance with the Dutch Alcohol Licensing and Catering act]. Utrecht: STAP.

Johnston, L.D., O'Malley, P.M., \& Bachman, J,G. (2000). The monitoring the future national survey results on adolescent drug use: Overview of key findings, 1999. NiH publ. no. 00-4690. National Institute on Drug Abuse, Rockville, MD.

Holder, H.D., Saltz, R.F., Grube, J.W., Treno, A.J., Reynolds, R.I, Voas, R.B., \& Gruenewald, P.J. (1997) Summing up; lessons from a comprehensive community prevention trial. Addiction, 92, 293-302.

Komro, K.A., Hu, F.B., \& Flay, B.R. (1997). A public health perspective on urban adolescents. In Walberg, H.J., Reyes, O., and Weissberg, R.P. (eds.), Children and youth: Interdisciplinary perspectives. Sage, Thousand Oaks, CA, pp. 253-298.

Kosterman, R., Hawkins, J.D., Gul, J., Catalano, R.F., \& Abbott, R.D. (2000). The dynamics of alcohol and marijuana initiation: patterns and predictors of first use in adolescence. American Journal of Public Health, 90, 360-366.

Lemmers, L., Willems, Y., Thijssen, P., \& Osterman, C. (1998). Evaluatie zomercampagne 1997 Lazer op met je zatte kop' [Evaluation summer campaign 1997 'Bugger off with your boozy talk']. Woerden: NIGZ.

Ministry of Health, Welfare and Sports (2007, June 1). Gemeenten laten bes/issen over leeftijdsgrens alcohalverkoop [Allowing municipalities to decide on a age limit for alcohol sales]. Retrieved from http://www.minvws.nl/nieuwsberichten/vgp/2007/gemeenten-leeftijd-alcohol.asp

Ministry of the Interior and Kindom Relations (2007, November 6). Toespraak minister Ter Horst bij ondertekening convenant Noord-Holland Noord [Speech given by minister Ter Horst at the signing of a covenant for the North of North Holland]. Retrieved from http://www.minbzk.nl/actueel/toespraken/109326/toespraak-minister_2

Municipality Schouwen-Duiveland (2001). Hoe eerder, hoe beter. Gezondheidsbeleid $2002-2005$ [The earlier, the better. Health policy 2002-2005]. Zierikzee: Author.

Municipality Schouwen-Duiveland (2004). Startnotitie integraal veligheldsbeleid Schouwen-Duiveland 2004-2007; Alcohol en wapenverbod 2004 o.b.v. art. 2.4.8. en 2.8.4a APV [Start memorandum integrated safety policy 2004-2007. Alcohol and weapon ban 2004, based on article 2.4.8 and 2.8.4a of local regulations]. Zierikzee: Author.

Perry, C.L. (1999). Creating health behavior change: How to develop community-wide programs for youth. Sage, Thousand Oaks, CA.

Perry, C.L., Williams, C.L., Komro, K.A., Veblen-Mortenson, S., Stigler, M.H., \& Munson, K.A. et al. (2002). Project Northland: long-term outcomes of community action to reduce adolescent alcohol use. Health Education Research, 17(1), 117-132.

Pos, S., Knibbe, R. \& Lemmers, L. (2001). Alle dagen feest? Een kwalitatieve studienaar omgevingsinvloeden op het alcoholgebruik van jongeren op vakantie [Party everyday? A qualitative study of environmental influences on young people's alcohol use on holiday]. Woerden/Maastricht: NIGZ/UM.

Stafström, M., Östergren, P., Larsson, S., Lindgren, B., Lundborg, P. (2006). A community action programme for reducing harmful drinking behavior among adolescents: the Trelleborg Project. Addiction, 101, 813-823.

Toomey, T.L., Wagenaar, A.C., Gehan, J.P., Kilian, G., Murray, D.M., \& Perry, C.L. (2001). Project ARM: Alcohol risk management to prevent sales to underage and intoxicated patrons. Health Education \& Behavior, 28(2), 186-199. 
Treno, A.J., Gruenewald, P.J., Lee, J.P., \& Remer, L.G. (2007). The Sacramento Neighbourhood Alcohol Prevention Project: Outcomes from a Community Prevention Trial. Journal of Studies on Alcohol and Drugs, 68 (2), 197-207.

Van de Hoef, P., Lemmers, L. \& Knibbe, R.A. (2001). Jongeren, alcohol en vakantie: Kwantitatieve studie naar het alcoholgebrulk van jongeren op vakantie in Nederland en Spanje IYoung people, alcohol, and holiday: A quantitative study on the alcohol use of youngsters on holiday in the Netherlands and Spain]. Woerden: NIGZ.

Van de Luitgaarden, J., Thush, C., Wiers, R.W., \& Knibbe, R.A. (2008). Prevention of alcohol problems in Dutch youth: Missed opportunities and new developments. Evaluation and the Health Professions, 31, 167-181.

Van de Luitgaarden, J., Wiers, R.W., Knibbe, R.A., \& Candel, M.J.J.M. (2007). Single-session expectancy challenge with young heavy drinkers on holiday. Addictive Behaviors, 32, 2865-2878.

Wagenaar, A.C., Murray, D.M., Wolfson, M., Forster, J.L., \& Finnigan, J.R. (1994). Communities Mobilizing for Change on Alcohol: design of a randomized community trial. Journal of Community Psychology (CSAP Special Issue), 79-101.

Wagenaar, A.C., Toomey, T.L., Erickson, D.J. (2005). Preventing youth access to alcohol: Outcomes from a multi-community time-series trial. Addiction, 100, 335-345

Yin, R.K. (2003). Case study research: Design and methods (3rd ed.). Thousand Oaks: Sage. 


\section{CHAPTER 8}

General Discussion 


\subsection{Introduction}

At the start of the research project reported on in this thesis, evidence suggested two intervention methods that could be used for prevention of excessive alcohol use in youth on holiday. The first was "community intervention" (Cl), an approach that focuses on changing the drinking environment, including the availability of alcohol through social and commercial sources. The second is "Expectancy Challenge" (EC, Darkes \& Goldman, 1993), an intervention aimed at reducing alcohol use through invalidating positive alcohol expectancies. Both intervention methods had never been applied to youth on holiday. Since the effectiveness of a program in one population and situation is no guarantee that it will also be effective in another, the decision was made to evaluate these interventions in the context of youth on holiday, after adapting them for use in this setting and population.

The main objective of this thesis is to explore the feasibility and effectiveness of both interventions in preventing heavy drinking in youth on holiday. The research project consisted of six parts, the objectives of which are summarized below:

- To evaluate the current Dutch situation of youth alcohol use, and provide insight into factors that hinder alcohol prevention in the Netherlands, as well as identifying new developments that may hold promise for the future.

- To evaluate if a single-session EC is effective in reducing university students' alcohol use through changing alcohol expectancies.

- To assess the feasibility of a single-session EC in a real-life prevention setting, with youth on holiday, while also exploring its effectiveness in changing expectancies and reducing alcohol consumption.

- To test the effectiveness of an optimized single-session EC in a larger sample of young men on holiday.

- To assess which contextual factors are related to young men's alcohol consumption on holiday.

- To evaluate compliance and effects of a community intervention based on selfregulation of stakeholders.

This chapter summarizes the main findings of the studies described in the previous chapters of this thesis. This is followed by some theoretical and methodological considerations. Furthermore, implications and recommendations for future research and policy are discussed.

\subsection{Main findings of the thesis}

To critically appraise the current Dutch situation of youth alcohol use (Chapter 2 , Van de Luitgaarden, Thush, Wiers, \& Knibbe, 2008), a literature study was done which revealed that Dutch alcohol laws are rather liberal and are hardly enforced. Youth drinking is culturally accepted, and alcohol availability for underage youth is 
high. Despite wide political interest in prevention of youth drinking, newly proposed alcohol laws are usually quickly dismissed. Politicians often prefer alcohol education over measures that target alcohol availability, however, there are indications that this may change. On the local level (e.g. region Eindhoven, Winterswijk) several initiatives have been taken to develop and implement a more comprehensive local alcohol policy primarily aimed at discouraging alcohol abuse among adolescents. Recently the national campaign against alcohol abuse has changed its focus from being directly aimed at young people to aiming at parents of adolescents, thereby stimulating that parents more closely monitor their children's alcohol use. It seems that the belief in alcohol education as the most important form of intervention has decreased at that there is more attention for the potential and the possibilities of prevention through changing the environment of drinkers.

The single-session EC that was done in the bar lab with university students (Chapter 3; Wiers, Van de Luitgaarden, Van den Wildenberg, \& Smulders, 2005) revealed a decrease in explicit positive arousal expectancies in men and women. In men (but not in women) there was a short-lived differential reduction in prospective alcohol use (significant in week 3 of the follow-up), and this reduction was partially mediated by the decrease in explicit positive arousal expectancies. These findings suggested that an EC had successfully changed explicit alcohol cognitions and that this may have beneficial effects on alcohol consumption of young men. Given these outcomes, it could be conclucled that a single-session challenge was feasible and (potentially) effective. However, it remained to be seen if the intervention could be realized and the findings generalized to other audiences and settings, and in particular to drinking youngsters in holiday situations.

In the first study to try the EC in young people $(N=170)$ on holiday (Chapter 4; Van de Luitgaarden, Wiers, Knibbe, \& Boon, 2006) the intervention was adapted for use in mixed-gender groups in a holiday setting. However, during the implementation phase several problems were encountered. Participants failed to show up for the intervention or could not be reached for follow-up, and there were several other circumstances which led to a lesser degree of standardization than desired by the authors. Nevertheless, the EC proved feasible in this setting, was well-received by youngsters and indications of effects of the EC on alcohol expectancies were found. No effects were found on alcohol use.

The next study implemented an optimized version of this EC on a larger scale in young males on holiday ( $N=264$, Chapter 5 ). The intervention was implemented correctly, and resulted in an increase in sedation expectancies in the EC group. Furthermore, the EC led to a differential reduction in alcohol consumption on a night out at the six-week posttest in the heaviest drinkers only. Surprisingly, the reduction in alcohol consumption on a night out was not mediated by the change in sedation expectancies (Van de Luitgaarden, Wiers, Knibbe, \& Candel, 2007). This raised questions on the working mechanisms of this intervention. If a change in alcohol expectancies was not the cause of the change in alcohol use, than the EC worked in a 
different way than we expected. As a result, it is unknown what elements of the intervention contributed to the reduction. Before applying the EC in an actual prevention context, it is important that this is known.

The next chapter (Chapter 6 ) reported on a study which assessed contextual factors related to young men's alcohol consumption on holiday. Results show that several contextual factors are positively associated with alcohol use (i.e. number of male friends in the peer group, switching age-identifying wristbands, and money). However, none of the measures designed to limit alcohol consumption were significant. This suggest that formal control on youth drinking needs to be improved.

An evaluation of the self-regulated alcohol community intervention done on Schouwen-Duiveland (Chapter 7) revealed several pitfalls of this intervention. Although all stakeholders agreed on the extent of the problems, most measures that would impact alcohol sales to youths were avoided when the covenant was drawn up. As a result, the final version involved mainly measures to reduce public nuisance, and only one new local measure that aimed to prevent alcohol sales to underage youth. Compliance with the covenant was not legally enforced, because of its voluntary nature. Although some positive changes were reported by local actors as a result of the covenant, it is important to recognize that separate, conflicting and irreconcilable interests were present, which hindered the chance of coming to effective measures. Because of the conflicts of interests, a fair amount of legal pressure is deemed necessary to ensure that measures that are agreed upon are also carried out.

\subsection{Methodological and theoretical considerations}

\subsubsection{Comparison with previous findings and current knowledge}

Community Intervention. Since this study started, new research findings have come to light. The effectiveness of specific environmental measures has been firmly established in the past few years. Social (e.g. the status of alcohol in the social environment), financial (e.g. affordability of alcohol), and physical (e.g. accessibility of alcohol) factors are known to affect drinking decisions, and are therefore often targeted by alcohol prevention policies. In this context, a distinction can be made between national alcohol laws, and local regulations regarding alcohol use.

In terms of national alcohol laws, Babor et al. (2003) and Room, Babor, \& Rehm (2005) pointed at the importance of public policy. Brand, Saisana, Rynn, Pennoni, \& Lowenfels (2007) did the same after comparing alcohol control policies of 30 countries. They found a link between the strength of alcohol control policies and amount of alcohol that was drunk. The most solid evidence exists for restrictions on alcohol availability (e.g., higher legal alcohol purchase age, server liability for damage caused by drunken patrons), higher alcohol prices, and measures against drunk driving (Brand et al., 2007). 
When it comes to local regulations on alcohol, community strategies such as checking alcohol vendors for compliance with underage laws, deterring adults from purchasing alcohol for minors, strengthening policies to detect and stop underage drinking parties, and instituting publicity for policies aimed at enforcement of laws have demonstrated that such efforts can reduce sales of alcohol to minors.

Results of the $\mathrm{Cl}$ described in this thesis should be interpreted as suggestions for measures to be used in future community interventions. In our study, the more money one was able to spend, the more alcohol one consumed. This is evidence for the role of alcohol affordability in determining consumption. If the price of alcohol is raised, the amount one consumes is likely to decrease. Young men who had traded wristbands to appear older for buying alcohol, drank significantly more on average. This points at the ease with which one can evade the rules as a factor influencing alcohol consumption. In general, the lower the perceived availability of alcohol, the lower alcohol consumption will be (Knibbe, Joosten, Derickx, Choquet, Morin, Monshouwer et al., 2005). The more male friends in the drinking group the more alcohol is consumed by group members individually. Drinks are offered frequently in large groups (e.g. another round of beer), therefore it is easy to obtain large amounts of alcohol. In summary the present study suggests that a community intervention aimed at reducing youths' alcohol use on holiday should be aimed at: 1 . increasing the price of alcohol, 2. Making it more difficult to obtain large amounts of alcohol, 3. Making it more difficult to evade alcohol laws and regulations, and 4. Decreasing the number of settings in which youth can drink to excess by setting rules on alcohol use.

In the $\mathrm{Cl}$ in our study, number of alcoholic drinks consumed per vacation day did not significantly differ between participants from the $\mathrm{Cl}$ municipality and those from the "no intervention" control community. Also, alcohol use in the $\mathrm{Cl}$ municipality was not lower during the holiday than it was two years earlier in the same community. The short time frame of this intervention, the limited measures agreed upon in the covenant, and the lack of enforcement of measures have probably contributed to the lack of results on alcohol consumption.

Expectancy Challenge. A few new EC studies have been published since the start of our study. At the time, there were indications that the EC was not effective in changing women's alcohol consumption (Dunn, Lau, \& Cruz, 2000; Corbin, McNair, \& Carter, 2001). Since then, several studies have replicated this finding, however some other studies did not. In a study by Musher-Eizenman and Kulick (2003) the EC was applied to college women, and although changes in expectancies were found, no reduction in drinking was achieved. These findings are in line with the ones we found when applying a single-session EC to male and female university students (Chapter 3; Wiers et al., 2005). Additionally, we noticed that women were much harder to recruit than men in a holiday setting (Chapter 4), and that they already drank much less on average (Van de Luitgaarden et al., 2006). After careful consideration, the decision was made to recruit only males for our further EC studies. However, recently, other authors successfully applied to EC to women. Wood, Capone, Laforge, Erickson, and Brand (2007) found similar intervention effects for men and women after their EC. Furthermore, a study by Lau-Barraco \& Dunn (2008) found 
that exposure to a single-session EC intervention led to significant decreases in alcohol expectancies and subsequent alcohol consumption in both genders at follow-up. The discussion about the effectiveness of the EC in women has not been closed, as new results continue to emerge.

Another point of discussion at the start of our study was if single-session EC's would be able to change alcohol use, or if a multiple-session protocol would be necessary for that. At the time, all studies demonstrating effects on alcohol consumption used a multiple-session protocol (e.g. Darkes \& Goldman, 1993; 1998, Dunn et al., 2000). In 2005, we were able to demonstrate limited effects on alcohol use as a result of a single EC session in young male university students (Wiers et al., 2005). In a later study, we found that the EC led to a differential reduction in alcohol consumption on a night out at the six-week posttest in the heaviest drinking voung men on holiday (Van de Luitgaarden et al., 2007). Recently, other authors have also found effects on alcohol use with a single-session protocol (Lau-Barraco \& Dunn, 2008).

Before our studies were conducted, no studies had been published that tested if changes in alcohol use after the EC were mediated by changes in alcohol expectancies. In the first study to test mediation (Wiers et al., 2005) there was a shortlived differential reduction in prospective alcohol use in men (significant in week 3 of the follow-up), and this reduction was partially mediated by the decrease in explicit positive arousal expectancies. A meditation effect was also found by LauBarraco \& Dunn (2008). Their mediation analyses provided evidence for significant indirect effects of the intervention on drinking outcomes through key alcohol expectancies. Specifically, global positive changes and social assertiveness expectancies were involved in mediating the effect of condition on typical weekly consumption and heavy episodic drinking frequency.

However, in an EC study that we carried out with youth on holiday (Van de Luitgaarden et al., 2007) the reduction in alcohol consumption on a night out (in the heaviest drinkers) was not mediated by the change in sedation expectancies that was found. What could have caused this null-finding on mediation? Perhaps, alcohol expectancies in youth on holiday are not stable, but vary over time, causing them to be less predictive of alcohol use in this specific population. Are there any indications that expectancies change that much in a short period of time? Our research paints a mixed picture: In one study (Van de Luitgaarden et al., 2007) the test-retest reliabilities of the sedation subscale were fairly low, however, other studies found adequate reliability, e.g. Wiers et al., 2005; Van de Luitgaarden et al., 2006). Another possible explanation is that other characteristics of the intervention caused the small change in heavy drinkers (e.g. seeing one's breath alcohol level after drinking). To ascertain that the EC decreases consumption by changing expectancies, further research on the EC should always include mediation analyses.

Another curious finding is that we unexpectedly found no cross-sectional correlations between alcohol expectancies and alcohol use in one EC study (Van de Luitgaarden et al., 2006). Pretest arousal and sedation expectancies did not correlate significantly with pretest alcohol consumption on a night out nor with pretest alcohol consumption on holiday. Several explanations for this finding were examined. 
First, as mentioned in the previous paragraph, the possibility that expectancies were not stable, or were measured inaccurately. However, test-retest scores on expectancies were sufficiently good in this study (Van de Luitgaarden et al., 2006), which suggests expectancies were measured reliably. Second, we examined if alcohol consumption was measured adequately. Test-retest reliabilities were satisfactory (in the control group), thus alcohol consumption was measured properly. Third, we ruled out high within-subject variation in alcohol use as an explanation, after we found indications that individual characteristics determine consumption rather strongly, irrespective of the situation. A fourth possibility is that expectancies may only correlate with drinking in populations with a wider range of alcohol use, while our participants were all heavy drinkers. In a later study (Van de Luitgaarden et al., 2007) we examined correlations between expectancies and alcohol use again, and found that pretest sedation expectancies correlated negatively with alcohol consumption on a night out, with total consumption from Monday through Friday and with consumption during the weekend. However, they did not correlate with alcohol use on holiday. Furthermore, all correlations between arousal expectancies and consumption were non-significant. Considering that many studies have confirmed a significant correlation between expectancies and consumption (Goldman et al., 1999), also with the instruments used here (Wiers et al., 2002, Wiers et al., 2005), we suspect specific features of the holiday situation or target group caused our results to differ.

Overall, only in our EC study in the surrogate bar (Wiers et al., 2005) were results as we expected. Studies in which the EC was applied to youth on holiday (Van de Luitgaarden et al., 2006, 2007) only partly confirmed our hypotheses.

\subsection{Limitations of the study}

Most limitations have been mentioned in the separate chapters. However, some issues remain to be addressed. These are related to design of the main study and choice of outcome measures.

\subsubsection{Design}

A limitation of the study is that we had to deviate from the original design (discussed in Chapter 1) because it proved difficult to find a suitable "communityintervention only" community, and the chosen community later dropped out. As a result, we were unable to analyze the effect of only a $\mathrm{Cl}$. We have tried to compensate for this by using data of the community in which both a $\mathrm{Cl}$ and $\mathrm{EC}^{\prime}$ s took place, before the start of the EC's. These alcohol use data were compared to alcohol use data collected in the same community in previous years.

We also deviated from the original time planning of the $\mathrm{Cl}$ part of the project, because it took longer than expected to find a $\mathrm{Cl}$ worker. As a result, the $\mathrm{Cl}$ could onty be started 5 months prior to the summer (when data had to be collected). As an optimal $\mathrm{Cl}$ requires trust, and building trust requires time, this short time frame 
may have influenced which measures were considered achievable by stakeholders, as well as stakeholder commitment.

Another limitation is that in several studies (e.g. Van de Luitgaarden et al., 2007), our sample did not include any women. Therefore, we were unable to come to definite conclusions on the effect of the interventions on female participants.

\subsubsection{Limitations in outcome measures}

In our EC studies, we used a modified version of the Visual Analogue Scale of arousal-sedation expectancies (Wiers, Van Woerden, Smulders, \& DeJong, 2002). This questionnaire was chosen because it specifically targeted high dose expectancies, and had satisfactory internal consistencies as well as test-retest reliabilities. Nevertheless, it would have been a good idea to include another expectancy questionnaire for comparison of results (e.g. the Dutch Alcohol Expectancy Questionnaire, VAV). However, an increase in the demands posed to participants could have led to increased attrition.

Furthermore, we used self-reported data in our studies. This may have introduced a potential source of bias. However, studies show that errors in reporting alcohol consumption are usually linear (Lemmens, Knibbe, \& Tan, 1988) and the same for respondents in both the EC and control condition. Moreover, the most important measures in our studies were of recent consumption (e.g. yesterday, the past weekend), which is likely to be reported more accurately than consumption that took place a longer time ago. Furthermore, we believe that young people themselves are likely to be the best (and sometimes only) witness to their own behavior.

Another limitation pertains to attrition of subjects from the studies. In one study (Chapter 4, Van de Luitgaarden et al., 2006), the level of attrition was very high, thus reducing the statistical power to find effects on outcome measures. This was due to several factors: youth on holiday often did not take appointments very seriously, no agendas were kept, and the EC location was far from the camping site. In later studies we were able to prevent these problems by reducing the time interval between recruitment and intervention, and driving participants to the intervention location.

The study reported on in Chapter 4 had an additional problem: Many participants filled out questionnaires inaccurately. Many found it difficult to remember the number of standard glasses they had drank (as most of them mainly drank bottles or cans of beer). This may have affected measurement of our main outcome measure (alcohol use). In later studies, we ensured that 'peers' were available to assist participants in filling out the questionnaire, and to briefly check the questionnaires for completeness. 


\subsection{Conclusion and recommendations}

\subsubsection{Expectancy Challenge}

The aim of the EC's with young men on holiday was to reduce their alcohol use in this context. Unfortunately, youth participating in the EC did not decrease their alcohol consumption during the holiday. Furthermore, the costs of conducting the EC are high (see Van de Luitgaarden et al., 2006) in both an absolute and relative sense, as relatively few people are reached through a rather elaborate effort. It may be that the EC is not particularly suited for application with young people on holiday. For this reason, implementation of this intervention in this situation does not seem useful at this point in time.

Nevertheless, studies by other authors found significant effects of the EC on alcohol use, and in two of the EC studies in this thesis, small favorable effects on alcohol use outside the holiday period were found as well. Therefore, the EC should not be written off, but instead, it would be a good idea to test this intervention in other preventative contexts, in which multiple sessions and a longer follow-up period are possible (e.g. in the context of social work, performed with youth at risk in a community centre, or as part of school curricula). As general alcohol consumption has been shown to be one of the main predictors of alcohol consumption on holiday, the mainstreaming of expectancy challenges into secandary school curricula might also contribute to a decrease in alcohol consumption on holiday.

One important point that future research into the effects of the EC needs to address is if mediation is present. In our study with university students that took place in the bar lab (Wiers et al., 2005), partial mediation was established. However, in another study in which the EC was applied to young males on holiday (Van de Luitgaarden et al., 2007), a lack of mediation was found, which suggested that the 'active ingredients' in the EC were not known. Furthermore, all participants in the studies in this thesis were heavy drinkers. It is possible that expectancies correlate with drinking only in study populations with a wider range of alcohol use. Further studies should test the EC in populations that include both light and heavy drinkers. A specific point of attention should be whether in such a population the heaviest drinkers are influenced by the EC as well.

Another direction for future research is to study if additive effects are present if an EC is combined with Motivational Interviewing (MI). To date, only Wood et al. (2007) have tested this. They found that, although both EC and BMI reduced alcohol use, there was no evidence for an additive effect of combining these interventions. In conclusion, the EC is still a promising intervention, but perhaps it is better suited to other prevention contexts. Additionally, we doubt the effect of an EC in an environment in which alcohol availability and drinking possibllities are unlimited.

\subsubsection{Community Intervention}

The $\mathrm{Cl}$ in the present study was different from those conducted elsewhere, because it was a voluntary, self-regulated agreement, and its measures were mainly aimed at a temporary population. Although this form of $\mathrm{Cl}$ was acceptable to nearly all 
stakeholders, it also gave local actors the chance to opt for less ambitious measures (that did not influence their own business), and the possibility to avoid enforcement of measures. The short time frame in which stakeholders were urged to decide on the contents of the covenant, may have contributed to the small number of new local measures that were taken.

$\mathrm{Cl}^{\prime}$ s need to involve more than one entity (e.g., police and alcohol sellers), take place in a variety of settings (e.g., camping site and bars), and be maintained for a longer period of time. In our study, those aspects of the $\mathrm{Cl}$ could be improved. Although camping attendants took on some new measures, alcohol availability in bars was still high, and age identification was not always checked. Alcohol sellers did not take on new measures, apart from the use of age-bracelets, which were not distributed as widely as planned. Many young people still drank alcoholic beverages in the public space (e.g. on the beach), and continuance of the $\mathrm{Cl}$ was unclear because funding for the project manager ended. The lack of enforcement of measures probably contributed to the lack of effects on youth alcohol use during the holiday as well.

One of the problems Dutch Cl's on alcohol face is that alcohol policies are enforced by the Food and Consumer Product Safety Authority (FCPSA), which is a national authority with very limited manpower. If the local police are given jurisdiction to enforce alcohol laws, compliance may be improved. A pilot study is being undertaken in several communities to test this proposal in practice.

\subsubsection{Closing remarks}

It can be concluded from this thesis that although the EC did succeed in influencing (sedation) expectancies and in that sense can be promising, it has so far not been able to significantly influence alcohol consumption of youth on holiday. However, interventions that are geared towards influencing contextual factors become increasingly relevant as alternative and potentially complementary strategies. Alcohol consumption of youth on holiday could likely be influenced through changing the context in which drinking takes place. Contextual factors could be targeted with policy measures that aim to reduce the availability of alcohol to young people. However, the presented implementation study of a Community Intervention is illustrative of the dominant Dutch political preference for governmental non-intervention and voluntarism over regulation and enforcement. It also illustrates the difficulties and subsequent ineffectiveness associated with such self-regulation and voluntary agreements between those involved. Yet as long as compliance with laws and regulations is inadequately enforced, the possibilities of decreasing alcohol consumption are not sufficiently exhausted and young people will be suffering from adverse effects of excessive alcohol use that could likely have been prevented. 


\section{References}

Babor, T., Caetano, F., Casswell, S., Edwards, G., Giesbrecht, N., Graham, K. et al., (2003) Alcohol: No ordinary commodity-A consumer's gulde to public policy. Oxford, U.K.: Oxford University Press.

Brand, D.A., Saisana, M., Rynn, L.A., Pennoni, F., \& Lowenfels, A.B. (2007). Comparative analysis of alcohol control policies in 30 countries. PLOS Medicine, 4(4): e151. Retrieved June 7, 2007, from http://www.medscape.com/viewarticle/556169

Corbin, W.R., McNair, L.D. \& Carter, J.A. (2001). Evaluation of a treatment-appropriate cognitive intervention for challenging alcohol outcome expectancies. Addictive Behaviors, 26, $475-488$.

Darkes, J. \& Goldman, M. S. (1993). Expectancy challenge and drinking reduction: experimental evidence for a mediational process. Journal of Consulting and Clinical Psychology, 61, 344-353.

Darkes, 1., \& Goldman, M.S. (1998). Expectancy Challenge and drinking reduction: Process and structure in the alcohol expectancy network. Experimental and Clinical Psychopharmacology, 6, 64-76.

Dunn, M. E., Lau, H. C. \& Cruz, I. Y. (2000). Changes in activation of alcohol expectancies in memory in relation to changes in alcohol use after participation in an expectancy challenge program. Experimental and Clinical Psychopharmacology, 8, 566-575.

Corbin, W.R., MCNair, L.Q. \& Carter, J.A. (2001). Evaluation of a treatment-appropriate cognitive intervention for challenging alcohol outcome expectancies. Addictive Behaviors, 26, $475-488$.

Knibbe, R.A., Joosten J., Derickx, M., Choquet, M., Morin, D., Monshouwer, K., et al. (2005). Perceived availability of substances, substance use and substance-related problems: a cross-national study among French and Dutch adolescents. Journol of Substance Use, 10(2-3), 151-163.

Lau-Barraco, C. \& Dunn, M.E. (2008). Evaluation of a single-session expectancy challenge intervention to reduce alcohol use among college students. Psychology of Addictive Behaviors, 22(2), 168-175.

Lemmens, P., Knibbe, R. A., \& Tan, F. (1988). Weekly recall and dairy estimates of alcohol consumption in a general population survey. Journal of Studies on Alcohol, 49(2), 1.31-135.

Musher-Eizenman, D. R. \& Kulick, A. D. (2003). An alcohol expectancy-challenge prevention program for at-risk college women. Psychology of Addictive Behaviors, 17, 163-166.

Room, R., Babor, T., and Rehm, J. (2005). Alcohol and public health. Lancet, 365, 519-530.

Van de Luitgaarden, J., Wiers, R.W., Knibbe, R.A. and Boon, B.J. (2006). From the laboratory to real life: A pilot study of an expectancy challenge with 'heavy drinking' young people on holiday. Substance Use and Misuse, 41, 353-368.

Van de Luitgaarden, J., Wiers, R.W., Knibbe, R.A., \& Candel, M.J.J.M. (2007). single-session Expectancy Challenge with young heavy drinkers on holiday. Addictive Behaviors, 32, 2865-2878.

Van de Luitgaarden, J., Thush, C., Wiers, R.W., \& Knibbe, R.A. (2008). Prevention of alcohol problems in Dutch youth: Missed opportunities and new developments. Evaluation and the Health Professions, 31, 167-181.

Wiers, R.W., Van de Luitgaarden, J., Van den Wildenberg, E., \& Smulders, F.T.Y.(2005). Challenging implicit and explicit alcohol-related cognitions in young heavy drinkers. Addiction, 100, 806-819.

Wlers, R.W., Van Woerden, N., Smulders, F. T. Y., De Jong, P. J. (2002). Implicit and explicit alcohol related cognitions in heavy and light drinkers. Journal of Abnormal Psychology, 111, 648-658.

Wood, M.D., Capone, C., Laforge, R., Erickson, D.J., \& Brand, N.H. (2007). Brief motivational intervention and alcohol expectancy challenge with heavy drinking college students: A randomized factorial study. Addictive Behaviors, 32, 2509-2528. 


\section{Summary}

On holiday, many adolescents and young adults drink much more than they regularly do. During a one to two week period, young men often drink as much as 22 standard drinks a day. This pattern of excessive alcohol use causes several problems, such as black-outs, fights, alcohol poisonings, traffic accidents, and unsafe sex. Several factors contribute to this drinking behaviour: absence of parents, presence of a large group of (male) friends, and the feeling of being in a "time-out" situation, in which normal behavioral rules do not apply, and school and parents are far away. With no one to supervise them, no social responsibilities and few activities to be done, drinking is the main pastime.

For many years, an alcohol education campaign took place in Dutch seaside communities during the summertime. Although the campaign resulted in greater awareness of the risks of excessive alcohol use among youth (Lemmers et al., 1998), their alcohol consumption on vacation did not decrease. Therefore, Maastricht University and NIGZ started a project to test two intervention methods in this population: community intervention $(\mathrm{Cl})$ and expectancy challenges $(\mathrm{EC})$. The studies in this thesis aimed to evaluate both the feasibility of both types of interventions in this setting, and the effects on young people's alcohol use on holiday.

Chapter 1 presents an overview of the extent of youth alcohol use, and its consequences. Furthermore, it provides background information on community interventions and expectancy challenges. The design of the main study is discussed, as well as the objectives of this thesis.

Chapter 2 provides a literature-based analysis of the current Dutch situation of youth alcohol use. Attention is given to cultural factors that influence youth drinking, alcohol policies, enforcement of alcohol laws, interventions, as well as to the current political climate for prevention of alcohol problems. Furthermore, the degree to which interventions that have a solid international research base are translated into effective measures in this country is discussed.

Chapter 3 reports on the effects of a single-session EC that took place in the university's bar-laboratory. Ninety-two heavy drinking college and university students (half women) were randomly assigned to the EC or control-condition (a sham alcoholexperiment in the same bar-lab). The effects of the EC on arousal and sedation expectancies and alcohol use were studied. Alcohol use was assessed prior to the experiment and during a one month follow-up. The EC resulted in decreased explicit positive arousal expectancies in men and women alike. In men but not in women there was a short-lived reduction in alcohol use (significant in week 3 of the followup), and this reduction was partially mediated by the decrease in explicit positive arousal expectancies. These findings suggest that the EC successfully changed ex- 
plicit alcohol cognitions and that this may have had short-lived beneficial effects in heavy drinking young men.

In Chapter 4, the feasibility of a single-session EC in a real-life prevention setting, with youth on holiday was assessed. In this study the EC was adapted for use in mixed-gender groups in a holiday setting and its feasibility tested in camping resorts in the Netherlands where a lot of binge-drinking takes place. The intervention itself took place in community centers and bars. Male and female participants $(N=170$, mean age 18.8 years) were randomly assigned to an EC or to an assessment-only control group. Alcohol expectancies and alcohol use (on holiday, and retrospectively prior to the holiday) were assessed one day before the intervention. Follow-up measurement of alcohol expectancies and alcohol use took place the day after the intervention, and six weeks later (via telephone). Although the study was hampered by recruitment difficulties, the EC proved feasible in this setting, was well-received by youngsters and there were some indications of effects on alcohol expectancies. No effects were found on alcohol use.

In Chapter 5 the effectiveness of an optimized single-session EC was tested in a larger sample of young men on holiday. We studied if changes in explicit alcohol expectancies and alcohol use occurred in the intervention group both during the holiday and after the holiday, as compared to a control group which did not take part in the EC. Alcohol expectancies and consumption were assessed with paper and pencil measures prior to the intervention $(N=301)$ and two days afterwards (EC: $n=$ 178; controls: $n=86$ ). Six weeks after the EC, participants were interviewed by telephone. The intervention resulted in an increase in sedation expectancies in the EC group. Alcohol use on holiday did not decrease. The EC did lead to a differential reduction in alcohol consumption on a night out at the six-week posttest in the heaviest drinkers only. However, this reduction in alcohol consumption on a night out was not mediated by the change in sedation expectancies. These findings suggested that further research on the mechanisms of change is necessary before a single-session EC may be used in a real-life prevention setting.

Chapter 6 reports on contextual factors related to young men's alcohol consumption on holiday. A stepwise regression analysis was used to determine the relevance of these factors in a sample of 492 young men (age 16-24) on vacation in seaside camping resorts in the Netherlands. Several types of contextual factors (e.g. age checks, peer group), demographic factors, as well as alcohol consumption in daily life and on vacation were assessed. Results show that several contextual factors are positively associated with alcohol use (i.e. number of male friends, switching ageidentifying wristbands, and money). However, none of the measures designed to limit consumption was significant. This suggests that formal control on youth drinking needs to be improved.

In Chapter $\mathbf{7}$ a community intervention based on self-regulation of stakeholders is evaluated. The community intervention was initiated in Schouwen-Duiveland, a 
community that attracts a large number of young, heavily drinking tourists every year. To battle youth alcohol abuse in the tourist season, a voluntary covenant which included several measures was signed. This covenant of parties concerned was evaluated on the basis of several types of data: questionnaires administered to young men on holiday $(N=191)$, observations carried out by trained 'peers', nuisance questionnaires among city centre residents $(N=121)$, and interviews with local actors. Stakeholders avoided measures that would impact alcohol sales to youths when the covenant was drawn up. As a result, measures agreed upon were fairly limited. Additionally, no provisions were made to ensure compliance of stakeholders with any of the measures. Therefore, it is not surprising that lack of compliance with measures restricting availability of alcohol continued to be a key problem.

Finally, in Chapter $\mathbf{8}$, the main results are put in a broader perspective. First, the main findings of the thesis are summarized. Then, the findings in this thesis are compared with previous findings and current knowledge. Overall, it is concluded that the EC does not reduce alcohol use in youth on holiday. However, interventions that are geared towards influencing contextual factors (such as $\mathrm{Cl}^{\prime}$ ) continue to be promising when policy measures aim to reduce the availability of alcohol to young people, and enforcement of measures is a priority. Furthermore, this chapter provides limitations of the studies, as well as implications and recommendations for alcohol prevention practice. Directions for further studies are also explored. 


\section{Samenvatting}

Op vakantie drinken veel adolescenten en jongvolwassenen veel meer dan ze gebruikelijk doen. Tijdens een periode van één à twee weken drinken jonge mannen gemiddeld 22 standaardglazen alcohol per dag. Dit patroon van excessief alcoholgebruik veroorzaakt diverse problemen, zoals black-outs, vechtpartijen, alcoholvergiftiging, verkeersongevallen en onveilige seks. Meerdere factoren dragen bij aan dit drinkgedrag: afwezigheid van ouders, aanwezigheid van een grote groep (mannelijke) vrienden en het gevoel zich in een time-out situatie te bevinden waarin school en ouders ver weg zijn en de gebruikelijke gedragsregels niet gelden. Zonder supervisie, zonder sociale verantwoordelijkheden en met weinig te doen is drinken het belangrijkste tijdverdrijf.

Reeds vele jaren vond er gedurende de zomer een alcoholvoorlichtingscampagne plaats in Nederlandse badplaatsen. Hoewel de campagne ertoe leidde dat jongeren zich meer bewust waren van de risico's van excessief alcoholgebruik (Lemmers et al., 1998), nam de alcoholconsumptie op vakantie niet af. Daarom zijn de Universiteit Maastricht en het NIGZ een project gestart om twee interventiemethoden in deze populatie te testen: De Community Intervention (Cl) en de Expectancy Challenge (EC). De studies in dit proefschrift zijn erop gericht om van beide methoden zowel de praktische haalbaarheid als de effecten op het alcoholgebruik van jongeren op vakantie te evalueren.

Hoofdstuk 1 verschaft een overzicht van de mate van alcoholgebruik door jongeren en de gevolgen daarvan. Daarnaast wordt er achtergrondinformatie over Community Interventions en Expectancy Challenges gepresenteerd. Ook het ontwerp van de hoofdstudie en de doelen van dit proefschrift worden besproken.

Hoofdstuk 2 geeft een op de literatuur gebaseerde analyse van de huidige Nederlandse situatie op het gebied van alcoholgebruik door jongeren. Er wordt aandacht besteed aan culturele factoren die hier invloed op hebben, maar ook aan alcoholbeleid, handhaving van alcoholwetten, interventies en aan het huidige politleke klimaat wat betreft preventie van alcoholproblematiek. Verder wordt besproken in welke mate op internationaal onderzoek gebaseerde interventies in Nederland vertaald worden in effectieve maatregelen.

Hoofdstuk 3 rapporteert over de effecten van een EC die bestond uit één sessie en die plaatsvond in het bar-laboratorium van de Universiteit Maastricht. Tweeënnegentig zwaar drinkende hogeschool- en universiteitsstudenten (voor de helft vrouwen) werden willekeurig aan de EC- of controleconditie (een nep-experiment in het zelfde bar-lab) toegewezen. De effecten van de EC op arousal- en sedation verwachtingen en op alcoholgebruik werden bestudeerd. Alcoholverwachtingen en alcoholgebruik werden gemeten onmiddellijk vóór deelname aan de EC en één maand na 
de interventie. De EC resulteerde in afgenomen expliciete, positieve arousal verwachtingen bij zowel mannen als vrouwen. Bij mannen werd een kortdurende reductie in alcoholgebruik gevonden (statistisch significant in de derde week van de follow-up) en deze reductie werd gedeeltelijk gemedieerd door de afname in expliciete positieve arousal verwachtingen. Dit resultaat werd niet bij vrouwen gevonden. Deze bevindingen suggereren dat de EC er in is geslaagd om expliciete alcoholcognities te veranderen en dat dit gedurende korte tijd voordelige effecten sorteerde voor zwaar drinkende jonge mannen.

In Hoofdstuk 4 is de haalbaarheid beoordeeld van de uitvoering van een EC bestaande uit één sessie in een echte preventiesetting met jongeren op vakantie. In dit onderzoek was de EC aangepast voor het gebruik in gemengde groepen van jonge mannen en -vrouwen in een vakantiesituatie en is deze vervolgens op haalbaarheid getest in Nederlandse badplaatsen waar veel excessief drankgebruik onder vakantievierende jongeren voorkomt. De interventie zelf werd uitgevoerd in gemeenschapshuizen en cafés. Mannelijke en vrouwelijke deelnemers ( $N=170$, gemiddelde leeftijd 18,8 jaar) werden willekeurig toegewezen aan ofwel de EC-groep, ofwel de groep waarin uitsluitend vragenlijsten werden afgenomen. Alcoholverwachtingen en alcoholgebruik (op vakantie en retrospectief voorafgaand aan de vakantie) werden één dag voor de interventie vastgesteld. Nameting van alcoholverwachtingen en alcoholgebruik vond de dag na de interventie plaats, evenals (telefonisch) zes weken erna. Ondanks problemen bij de werving van proefpersonen, bleek de EC haalbaar te zijn in deze setting. De interventie werd goed ontvangen door jongeren en er waren indicaties van effecten op alcoholverwachtingen. Op drankgebruik werden geen effecten gevonden.

In Hoofdstuk $\mathbf{5}$ wordt de effectiviteit van een geoptimaliseerde EC die bestaat uit één enkele sessie getest in een grotere steekproef van vakantie vierende jonge mannen. We bestudeerden of er veranderingen in expliciete alcoholverwachtingen en alcoholgebruik optraden tijdens en na de vakantie in de interventiegroep vergeleken met de controlegroep. Alcoholverwachtingen en -consumptie werden gemeten door middel van vragenlijsten $(N=301)$ voorafgaand aan de interventie en twee dagen later (EC: $n=178$; controls: $n=86$ ). Zes weken na de EC werden deelnemers opnieuw telefonisch ondervraagd. De interventie resulteerde in een toename van sedatie verwachtingen in de EC groep. De alcoholconsumptie op vakantie nam echter niet af. De EC leidde wel tot een differentiële reductie in alcoholconsumptie tijdens het uitgaan bij de nameting die zes weken na de interventie plaatsvond, echter dit gold alleen voor de zwaarste drinkers. Deze reductie in alcoholconsumptie werd niet gemedieerd door de verandering in sedatie verwachtingen. Deze bevindingen impliceren dat verder onderzoek nodig is naar de werkingsmechanismen van de EC voordat deze gebruikt kan worden in een echte preventiesetting.

Hoofdstuk 6 rapporteert over contextuele factoren die gerelateerd zijn aan de alcoholconsumptie van vakantievierende jonge mannen. Een stapsgewijze regressieanalyse werd gebruikt om de relevantie van deze factoren te bepalen in een steekproef 
van 492 vakantievierende jonge mannen (in de leeftijd van 16 - 24 jaar) in Nederlandse badplaatsen. Verschillende typen contextuele factoren (bijvoorbeeld leeftijdscontroles, vriendengroep), demografische factoren, alcoholconsumptie op vakantie en alcoholconsumptie in het dagelijks leven werden gemeten. De resultaten tonen aan dat verscheidene contextfactoren positief geassocieerd zijn met alcoholgebruik (bijvoorbeeld aantal mannelijke vrienden, het verwisselen van armbandjes die leeftijd aangeven en besteedbaar geld). Echter geen van de maatregelen om consumptie te beperken was significant. Dit impliceert dat formele controle op het alcoholgebruik van jongeren verbeterd moet worden.

In Hoofdstuk 7 wordt een op zelfregulering gebaseerde Community Intervention geëvalueerd. De Community Interventie was in gang gezet op Schouwen-Duiveland; een gemeenschap die grote groepen zwaar drinkende jonge toeristen aantrekt. Om drankmisbruik in het toeristenseizoen te bestrijden, werd er een vrijwillig convenant getekend waarin maatregelen vastgelegd waren. Dit convenant van betrokken partijen werd geëvalueerd op basis van verschillende soorten data: vragenlijsten ingevuld door jonge mannen op vakantie ( $N=191)$, observaties uitgevoerd door getrainde 'peers', overlastvragenlijsten ingevuld door bewoners van het centrum van het dorp $(N=121)$ en interviews met plaatselijke actoren. Bij het opstellen van het convenant vermeden belanghebbenden maatregelen die de alcoholverkoop aan jongeren zouden beïnvloeden. Als gevolg daarvan waren de maatregelen waarover overeenstemming bestond tamelijk beperkt. Bovendien werden er geen bepalingen in opgenomen die de naleving ervan door belanghebbenden verzekerden. Het is daarom weinig verrassend dat een gebrek aan naleving van maatregelen die de beschikbaarheid van alcohol beperkten een belangrijk probleem blijft.

Tenslotte worden in Hoofdstuk $\mathbf{8}$ de belangrijkste resultaten in een breder perspectief geplaatst. Eerst worden de belangrijkste bevindingen van dit proefschrift samengevat. Daarna worden de bevindingen vergeleken met eerdere bevindingen en de huidig beschikbare kennis op dit gebied. Alles overwegende wordt geconcludeerd dat de EC het alcoholgebruik van jongeren op vakantie niet beperkt. Interventies die gericht zijn op het beïnvloeden van contextuele factoren (zoals Community Interventies) blijven echter veelbelovend wanneer ze gestoeld zijn op beleidsmaatregelen die de beschikbaarheid van alcohol voor jongeren beperken, en handhaving van maatregelen prioriteit krijgt. Verder worden in dit hoofdstuk beperkingen van de studies benoemd, evenals implicaties en aanbevelingen voor praktische alcoholpreventie. Mogelijke richtingen voor nader onderzoek worden ook verkend. 


\section{Dankwoord}

Vele mensen hebben bijgedragen aan mijn proefschrift. Ik wil hen hiervoor graag hartelijk bedanken.

Als eerste wil ik graag mijn promotoren Ronald Knibbe en Reinout Wiers bedanken. Bedankt dat jullie me destijds de kans gaven om aan dit onderzoek te werken. Jullie zijn het beste team van begeleiders geweest dat een Aio zich maar kon wensen. Ik kon altijd bij jullie binnenlopen en jullie reageerden altijd snel op mijn e-mails. Jullie gaven me de ruimte om mijn eigen stempel te drukken, maar waren er ook met goede raad wanneer ik vastliep bij wat ik deed. Jullie commentaar was altijd waardevol en ik heb daardoor veel geleerd over met name statistiek en het schrijven van wetenschappelijke artikelen. Ik vond het heel fijn om met jullie samen te werken.

De leden van de beoordelingcommissie wil ik bedanken voor hun bereidheid zitting te nemen in de commissie en het concept proefschrift van commentaar te voorzien.

Dank ook aan de co-auteurs van de artikelen, met name aan Brigitte Boon voor haar bijdrage aan de pilot van de Expectancy Challenge studie en aan Math Candel voor zijn statistische ondersteuning.

Tijdens m'n AlO tijd was er een constante factor: Het wekelijkse AIO lunchoverleg bij Experimentele Psychologie met Reinout, Esther, Carolien, Katrijn en Tim. Het was fijn om laagdrempelig om input te kunnen vragen en daarnaast was het altijd erg gezellig en heb ik veel gelachen om de dingen (niet altijd werkgerelateerd) die ter sprake kwamen. De jaarlijkse barbecue bij Reinout was telkens weer een culinaire verrassing en ik heb ook enorm genoten van het congresbezoek in Santa Barbara in 2005 met jullie. Bedankt voor de tips, hulp en gezelligheid die jullie me gaven.

Ook aan ons alcoholclubje bij Medische Sociologie heb ik goede herinneringen. De heerlijke lunches thuis bij Ronald, Jan, Wendy, Jikke, Moniek en Daksha waren geweldig. En ook daar was het prettig om feedback te kunnen krijgen.

Op de afdeling bij Medische Sociologie voelde ik me goed thuis. De dagelijkse thee brul, de leuke gesprekken met collega's, de jaarlijkse uitjes, ik hoorde er helemaal bij en vond het dan ook wel jammer dat de afdeling werd opgesplitst en ik voor de laatste paar maanden van m'n aanstelling bij GVO werd geplaatst. Maar ook daar werd ik warm ontvangen, waarvoor dank.

Sommige collega's van het oude MedSoc wil ik nog even extra bedanken. Dan denk ik bijvoorbeeld aan Marjoke die altijd precies wist hoe je dingen moest regelen en die altijd klaar stond om hulp te bieden bij praktische zaken. Sommige collega's werden vriendinnen: Marja, Daksha, Moniek, Esther. We hadden het soms iets té 
gezellig, maar ik heb veel van jullie geleerd en jullie hebben er mede voor gezorgd dat ik altijd met veel plezier naar de uni ging.

Ook een bedankje voor Marja Vissers omdat ze vanalles rondom mijn proefschrift heeft geregeld, zoals het versturen naar de leescommissie. Ook alle andere collega's van het oude Medische Sociologie en van GVO: Bedankt!

De gemeenten Schouwen-Duiveland en Zandvoort, alsmede de leden van de alcoholbeleidsgroepen, wil ik hartelijk bedanken voor hun medewerking aan het onderzoek.

Ook zonder het NIGZ en de peers die de interventies op locatie uitvoerden en de vragenlijsten afnamen had het onderzoek nooit plaats kunnen vinden. In het bijzonder wil ik Martijn Planken, Tamara, Rob Bovens, Jan Huige en Annemarie Huiberts bedanken voor hun werk ten behoeve van het onderzoek. En natuurlijk wil ik ook de proefpersonen bedanken, die speciaal voor het onderzoek een ochtendje nuchter bleven!

Daarnaast wil ik MEMIC bedanken voor het invoeren van gegevens en het uitvoeren van de telefonische interviews.

Verder had ik dit proefschrift niet kunnen schrijven zonder de steun en hulp van mijn man Guido. Dit proefschrift heeft jou net zoveel energie gekost als mij. Door mijn werk kwam er qua kinderen veel op jou neer en qua huishouden ook het nodige. Omdat je zelf ook nog 3 banen hebt, weet ik dat dit voor jou ook bij tijden zwaar is geweest. Daarnaast was jij altijd de eerste die mijn artikelen las, nog voordat ik ze aan anderen durfde te laten lezen. Je gaf altijd goede adviezen en bent supergoed in problemen oplossen. Dus thanks!

Pap en Mam, jullie wil ik ook heel hartelijk bedanken. Jullie hebben me altijd gestimuleerd om een opleiding te volgen en om door te leren en hebben dat ook op alle manieren mogelijk gemaakt, door materieel te steunen tijdens mijn studle Psychologie en door regelmatig op de kinderen te passen wanneer ik moest werken terwijl er geen kinderopvang beschikbaar was. Dank jullie wel!

Verder wil ik mijn zusjes Mirre en Fiere bedanken dat ze mijn paranimf wilden zijn en alle andere vrienden en vriendinnen (o.a. Daksha, Mattijn, Marja, Peter, Rianne, Michiel, Tessie, David, Paul, Stefan), waarmee ik regelmatig cocktails drink en spellen speel. Onze gezellige avonden zorgden voor de afleiding die soms hard nodig was.

Last but not least, een bedankje voor Cosmo, Lente, Vega, en Desirée. Jullie zijn de meest geweldige kinderen die ik me maar had kunnen wensen en jullie zorgden ervoor dat ik m'n proefschrift zo snel mogelijk wilde afronden om meer bij jullie te kunnen zijn (:) 


\section{Curriculum vitae}

Jade van de Luitgaarden-Janssen was born on July 2 nd, 1979 in Heerlen, the Netherlands. She completed her secondary education (VWO) at Sint Michiel in Geleen. After her graduation in 1997, she studied Psychology at Maastricht University. In her Master thesis she studied the effectiveness of a single session Expectancy Challenge in heavily drinking university students.

After obtaining her Master of Science degree in 2001, Jade started working on her PhD project at the Faculty of Health, Medicine, and Life Sciences of Maastricht University in 2002. This project, which is described in this thesis, covered two intervention methods aimed at reducing alcohol use in youth on holiday. The feasibility and effectiveness of Expectancy Challenges and Community Interventions were studied in a vacation setting.

Since lanuary 2008 , she is a partner in the conference agency ECB, which organizes seminars on health and social care related topics. This job allows her to combine work with the care for her children. 


\section{Publications}

Van de Luitgaarden, J., Thush, C., Wiers, R.W., \& Knibbe, R.A. (2008). Prevention of alcohol problems in Dutch youth: Missed opportunities and new developments. Evaluation and the Health Professions, 31, 167-181.

Van de Luitgaarden, J., Wiers, R.W., Knibbe, R.A. and Boon, B.J. (2006). From the laboratory to real life: $A$ pilot study of an expectancy challenge with 'heavy drinking' young people on holiday. Substance Use and Misuse, 41, 353-368.

Van de Luitgaarden, J., Wiers, R.W., Knibbe, R.A., \& Candel, M.J.J.M. (2007). Single-session expectancy challenge with young heavy drinkers on holiday. Addictive Behaviors, 32, 2865-2878.

Van de Luitgaarden, J., Knibbe, R.A., \& Wiers, R.W. (in press). Adolescents binge drinking when on holi day: An evaluation of a community intervention based on self regulation. Substance Use and Misuse. 44.

Van de Luitgaarden, J., Knibbe, R.A., \& Wiers, R.W. (submitted). How easy is it to get drunk? Contextua factors related to young people's excessive alcohol use on holiday.

Wiers, R.W., Van de Luitgaarden, J., van den Wildenberg, E., \& Smulders, F.T.Y. (2005). Challenging impi. cit and explicit alcohol-related cognitions in Young heavy drinkers. Addiction, 100, 806-819. 